\title{
Design Automation and Layout Optimization of Analog Decoders
}

by

\author{
Xiaoning Li, B. Eng. \\ A thesis submitted to \\ the Faculty of Graduate Studies and Research \\ in partial fulfillment of \\ the requirements for the degree of \\ Master of Applied Science in Electrical Engineering \\ Ottawa-Carleton Institute for Electrical and Computer Engineering \\ Department of Systems and Computer Engineering \\ Carleton University \\ Ottawa, Ontario, Canada
}

November, 2006

(c) Copyright 2006, Xiaoning Li 


$\begin{array}{ll}\begin{array}{l}\text { Library and } \\ \text { Archives Canada }\end{array} & \begin{array}{l}\text { Bibliothèque et } \\ \text { Archives Canada }\end{array} \\ \begin{array}{l}\text { Published Heritage } \\ \text { Branch }\end{array} & \begin{array}{l}\text { Direction du } \\ \text { Patrimoine de l'édition }\end{array} \\ \begin{array}{l}\text { 395 Wellington Street } \\ \text { Ottawa ON K1A 0N4 }\end{array} & \begin{array}{l}\text { 395, rue Wellington } \\ \text { Ottana ON K1A ON4 } \\ \text { Canada Oa }\end{array}\end{array}$

Your file Votre référence ISBN: 978-0-494-26995-4 Ourfile Notre référence ISBN: $978-0-494-26995-4$

NOTICE:

The author has granted a nonexclusive license allowing Library and Archives Canada to reproduce, publish, archive, preserve, conserve, communicate to the public by telecommunication or on the Internet, loan, distribute and sell theses worldwide, for commercial or noncommercial purposes, in microform, paper, electronic and/or any other formats.

The author retains copyright ownership and moral rights in this thesis. Neither the thesis nor substantial extracts from it may be printed or otherwise reproduced without the author's permission.
AVIS:

L'auteur a accordé une licence non exclusive permettant à la Bibliothèque et Archives Canada de reproduire, publier, archiver, sauvegarder, conserver, transmettre au public par télécommunication ou par l'Internet, prêter, distribuer et vendre des thèses partout dans le monde, à des fins commerciales ou autres, sur support microforme, papier, électronique et/ou autres formats.

L'auteur conserve la propriété du droit d'auteur et des droits moraux qui protège cette thèse. $\mathrm{Ni}$ la thèse ni des extraits substantiels de celle-ci ne doivent être imprimés ou autrement reproduits sans son autorisation.
In compliance with the Canadian

Privacy Act some supporting forms may have been removed from this thesis.

While these forms may be included in the document page count, their removal does not represent any loss of content from the thesis.
Conformément à la loi canadienne sur la protection de la vie privée, quelques formulaires secondaires ont été enlevés de cette thèse.

Bien que ces formulaires aient inclus dans la pagination, il n'y aura aucun contenu manquant. 
I hereby declare that I am the sole author of this thesis.

I authorize Carleton University to lend this thesis to other institutions or individuals for the purpose of scholarly research

Signature

I further authorize Carleton University to reproduce this thesis by photocopying or by other means, in total or in part, at the request of other institutions or individuals for the purpose of scholarly research.

Signature 


\begin{abstract}
This thesis will discuss the VLSI implementation of analog LDPC decoders. The good performance of LDPC codes and their intrinsic parallel decoding algorithm makes them suitable for high performance decoder design. Analog circuits are chosen due to their simpler circuits, smaller number of interconnections (less area and power consumption), the disappearance of iterations (faster convergence and higher throughput) and lower noise generation. However, in analog LDPC decoder design, an effective VLSI implementation method with good results remains a serious challenge for two reasons: 1) traditional analog design flow is not suitable for large-scale analog design, and 2) lack of universal optimization methods

In this thesis, we propose an iterative optimization method that can be applied to an automatic analog design flow based on pre-built macro cells. This method simplifies the design process and optimizes the design result with the help of a placement and routing tool called First Encounter (FE). It is shown in this thesis total interconnection wire length can be used to evaluate the optimization result. In our case, with certain constrains (pre-built cells, parity-check matrix and preset layout configuration), the optimization aims to find an optimized position for each cell in order to reduce the total wire length. The final result shows that a $25 \%$ to $35 \%$ reduction in total interconnection wire length comparing to initial random placement can be achieved with this method. Unlike previous optimization methods, which are usually restricted to a certain type of code, this optimization method can accommodate different code types, and is more general.
\end{abstract}




\section{Acknowledgements}

This thesis would not have been possible without the support and help of many people. I will take this opportunity to express my sincere gratitude to all of them.

First, I should thank my supervisor, Dr. Amir H. Banihashemi. His earnest love for research, broad knowledge and insightful instructions inspired me during the research period. I should also thank Prof Plett, who has been actively involved in our group's research activities. His abundant experience in VLSI design helped a great deal during our analog decoder design.

Second, I am deeply indebted to my fellow students, who offered their help without hesitation. Principle among these were Hemati, Hua, Lam and Yuri; there are many others, too numerous to list.

And finally I owe much to my beloved family, especially my mother.

Again, thanks to all of you. 


\section{Table of Contents}

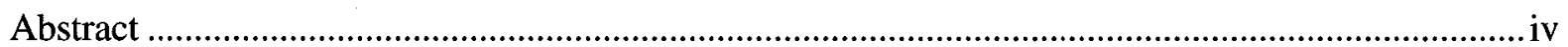

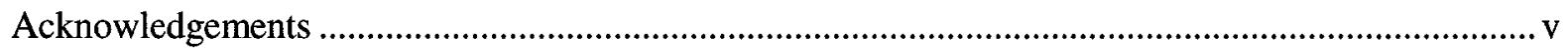

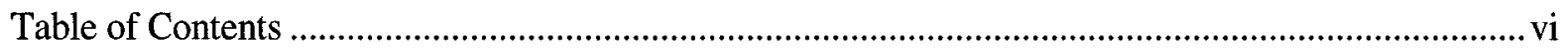

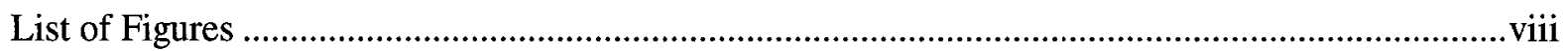

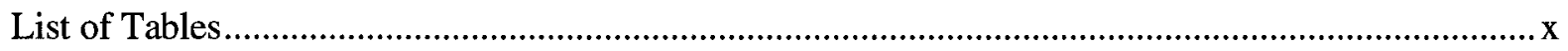

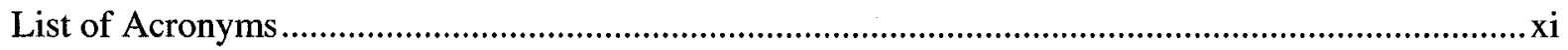

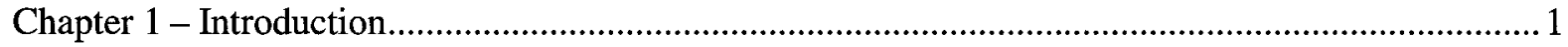

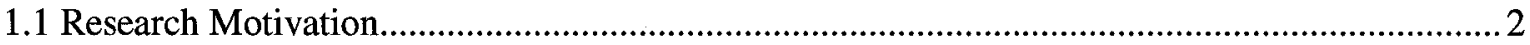

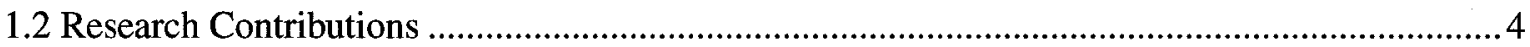

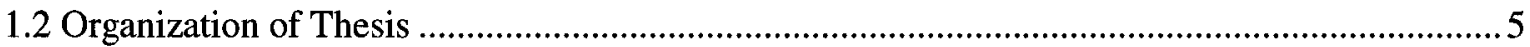

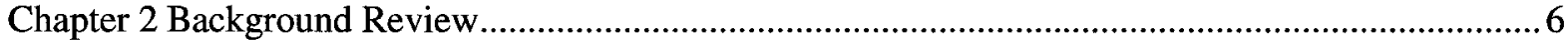

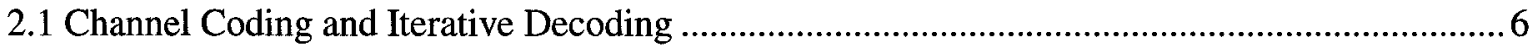

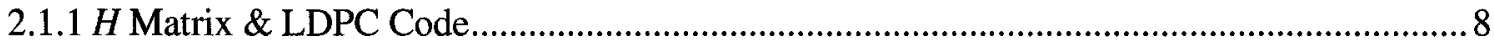

2.1.2 Graph Representation (Tanner Graphs) ............................................................................... 10

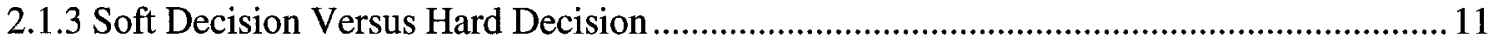

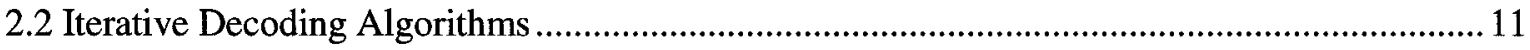

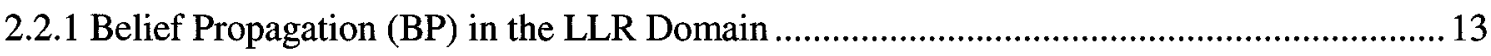

2.2.2 Min-Sum (MS) Algorithm in the LLR Domain ................................................................ 15

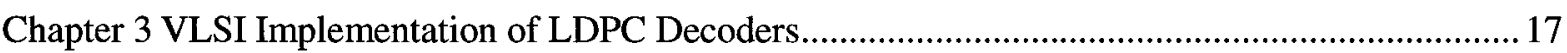

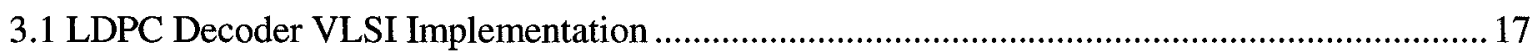

3.1.1 Review of Different Implementations of Iterative Decoding ......................................... 18

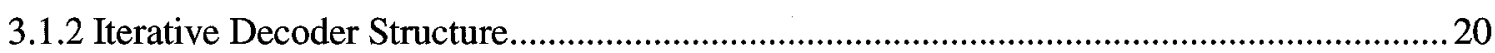

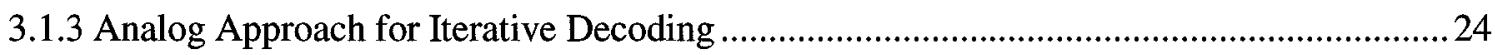

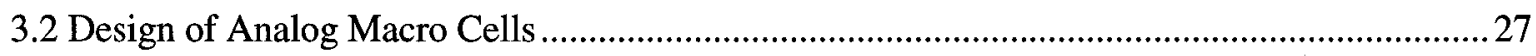

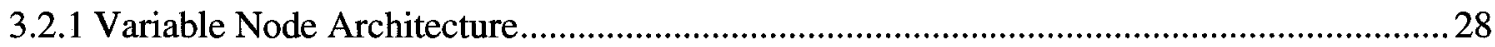

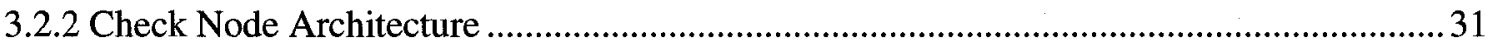

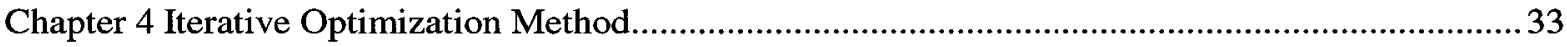

4.1 Current Optimization Methods for an Interconnecting Network and Our Design Flow ............36

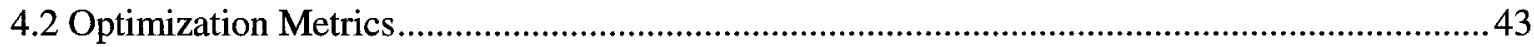

4.3 Input Files for FE (First Encounter) Generation ...................................................................51

4.3.1 Automatic Structural Verilog Code Generation .......................................................................51

vi 


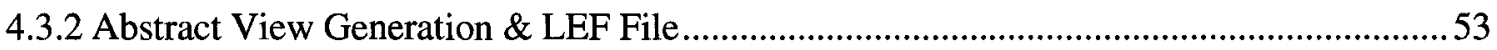

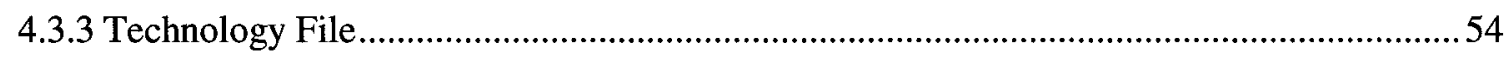

4.4 Placement \& Routing: Iterative Optimization Method............................................................54

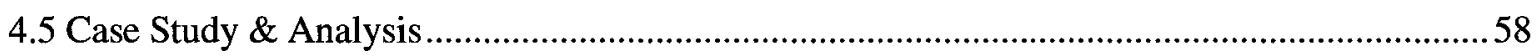

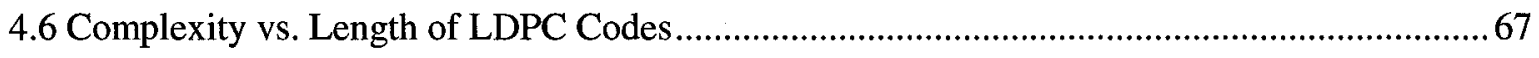

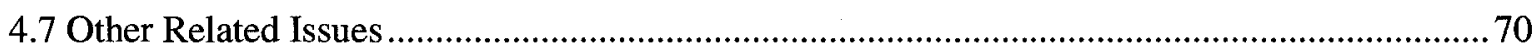

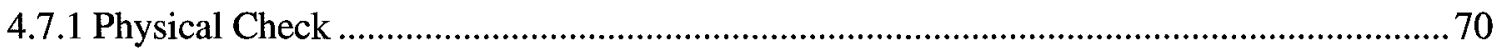

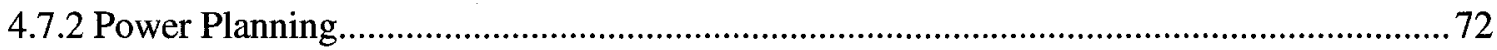

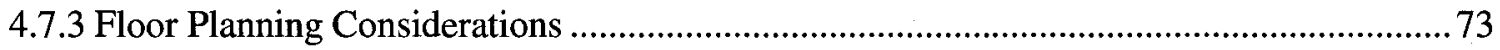

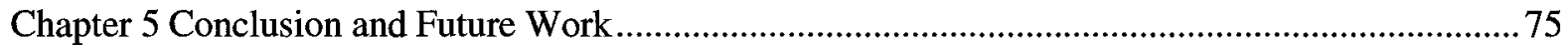

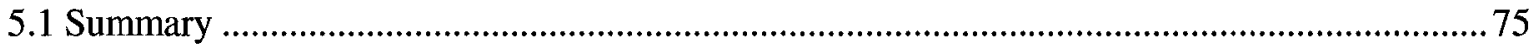

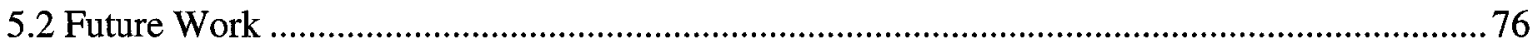

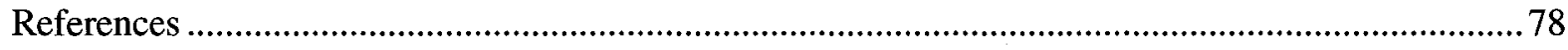

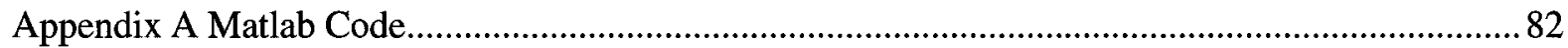

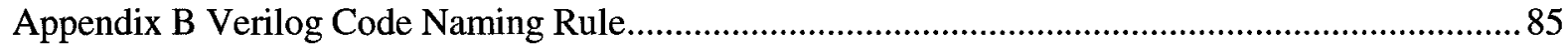

Appendix C Automatically Generated Verilog Code........................................................................ 86

Appendix D Partial Technology File ....................................................................................... 91

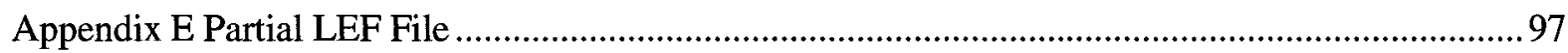

vii 


\section{List of Figures}

Figure 2.1 Simplified Classical Model of a Digital Communication System.......................

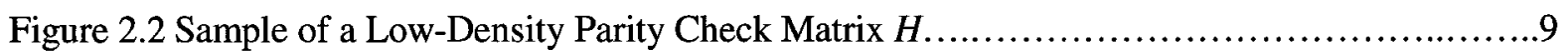

Figure 2.3 TG Representation of a LDPC Code with the $H$ Matrix from Figure 2.2 plus Information Flow in the Message-Passing Algorithm.

Figure 2.4 Simplified Tanner Graph Showing Message-Passing Scheme for Check

Node. .14

Figure 2.5 Simplified Tanner Graph Showing Message-Passing Scheme for Variable

Node. 14

Figure 3.1 Relative Comparisons Among Different Platforms for LDPC Decoder Implementation. .20

Figure 3.2 Full Parallel Structure of LDPC Decoder .22

Figure 3.3 Serial Structure of LDPC Decoder. .23

Figure 3.4 Current buffer with (1) n-type current mirror active

(2) p-type current mirror active .29

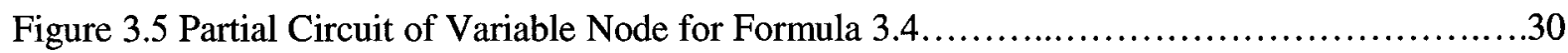

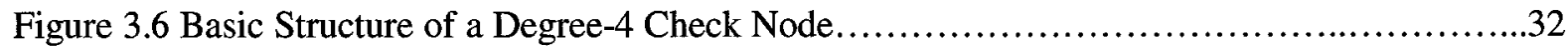

Figure 4.1 Traditional Digital Design Flow.... .35

Figure 4.2 Overview of Design Flow [18] Used to Automate the Construction of a Large-Scale LDPC Analog Decoder. .40

Figure 4.3 Design Flow Used to Automate the Construction of a Large Analog Decoder Suitable for an Iterative Optimization Process........................................................42

Figure 4.4 Capacitive Coupling Components...........................................44

Figure 4.5 Simple Lumped RC Model of an Interconnected Wire ..............................47

Figure 4.6 Distributed RC Ladder Network Model Consisting of N Equal Segments.................48

Figure 4.7 Sample $H$ Matrix...........................................................52

Figure 4.8 Iterative Optimization Method Using First Encounter ..............................57

Figure $4.9 \mathrm{H}$ Matrix of a $(32,8,10)$ Regular LDPC ........................................ 58

viii 
Figure 4.10 Layout of Analog Decoder for $(32,8,10)$ LDPC Code Following Traditional Design

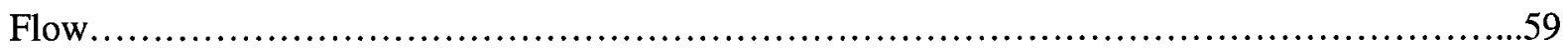

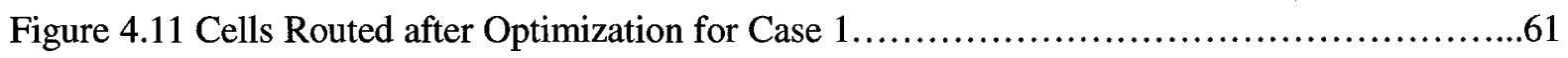

Figure 4.12 Wire Length vs. Iteration....................................................62

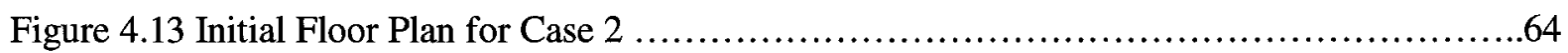

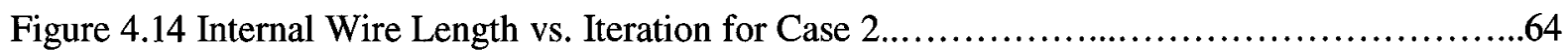

Figure 4.15 Internal Wire Length vs Iteration for Case 3.......................................65

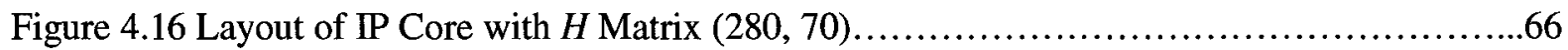

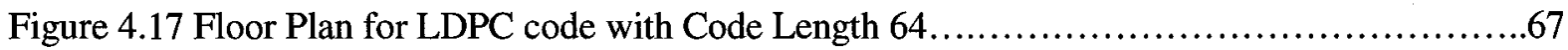

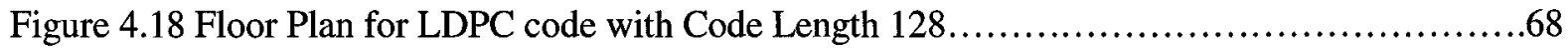

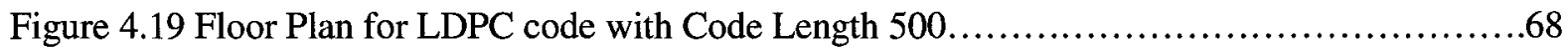

Figure 4.20 Complexity of Iterative Optimization vs. Code Length............................69 


\section{List of Tables}

Table 3.1 A Comparison of Different Platforms............................................23

Table 4.1 Utilization Rate for LDPC Parallel Decoders...........................................50

Table 4.2 Optimization Effect Comparison for Different Cases................................66 


\section{List of Acronyms}

ACS

ADC

ASTR

ASIC

AWGN

BER

BiCMOS

BJT

BP

DCVSL

DVC

DSP

FPGA

IC

IP

LDPC

LEF

LLR

MS

MSF

PE

PAE

RTAS

SP

VLSI

WTA
Add-Compare-Select

Analog to Digital Converter

Absolute Value and Sign to Real Converter

Application Specific Integrated Circuit Design

Addictive White Gaussian Noise

Bit Error Rate

Bipolar \& CMOS Transistors in the Same Silicon Process

Bipolar Junction Transistor

Belief Propagation

Differential Cascade Voltage Switch Logic

Digital to Analog Converter

Digital Signal Processing

Field Programmable Gate Arrays

Integrated Circuit

Intelligent Property

Low Density Parity Check Code

Library Exchange Format

Log-likelihood Ratio

Min-Sum Decoding Algorithm

Message Scaling Factor

Processing Element

Process Antenna Effect

Real to Absolute Value and Sign Converter

Sum-Product Decoding Algorithm

Vast Large Silicon Integrated Circuit

Winner-Take-All 


\section{Chapter 1 - Introduction}

Shannon introduced his famous theorem on channel coding in 1948 [1]. Since then, applications of information theory as well as communication technologies have experienced great progress. Shannon's theorem, which states that reliable communication is possible over noisy channels provided that the rate of communication is below a certain threshold called the channel capacity. The channel capacity can be approached by using appropriate encoding and decoding systems. This theorem provides an objective guide for code design. In this thesis, the term "encoding" will refer to the act of adding redundant bits to the data stream. This type of coding is generally called channel coding. The redundancy introduced by the coding scheme helps to decode received sequences by finding the best decision for the sent data.

Loosely speaking, the history of error-control coding can be divided into "pre-turbo code" and "postturbo code." Turbo codes and their ingenious iterative decoding scheme, devised in 1993 by French researchers Claude Berrou and Alain Glavieux, revolutionized this area [2]. Prior to their invention, it was impractical to get close to the theoretical performance limit promised by Shannon's theory.

The effort to come up with good coding schemes started a long time ago. In the early 1960s, Gallager introduced low-density parity check (LDPC) codes in his PhD thesis [3]. Because the computing power at that time was insufficient, the associated iterative decoding algorithms were generally impractical in spite of their powerful error-correcting capability. With the progress in computing power, interest in iterative decoding algorithms emerged after LDPC codes were forgotten for about 30 years. Since then, LDPC codes have received a great deal of attention due to their capacity to achieve near- 
Shannon limit error performance with low decoding complexity. It has been observed that LDPC codes with long block lengths outperform the best-known turbo code in similar situations. Currently the most powerful LDPC code known has a block length of $10^{7}$, rate $1 / 2$, and it achieves a performance of only $0.0045 \mathrm{~dB}$ away from the Shannon limit for a bit error probability (BER) of $10^{-6}$ [4]. The high performance puts LDPC codes in a promising position to become the standard for error control coding in cell phones, digital video broadcast (DVB), inter-planetary communication, etc. These standards include WiMax (IEEE802.16e) [41], Wireless LAN (IEEE802.11n) [42], Satellite Digital Broadcast HDTV (DVB-S2) [43], and 10Gbit/s Ethernet (802.3an) [44].

Over the past few years, the demand for efficient and reliable data transmission and data storage has increased significantly. This trend was accelerated by the emergence of high-speed data networks and high-density data storage in recent years. A wide range of research efforts have been made in the areas of LDPC code construction techniques [13] [15] [16], VLSI structure design for LDPC decoders [10] [27] [28], and design methodologies based on a variety of platforms for hardware implementation [14] [17] [18]. The aim of these research projects was to find optimized solutions under different situations, with trade-offs involving throughput, power consumption, chip size and design methodology.

\subsection{Research Motivation}

Signal transmission is always subject to noise and external disturbances. Therefore channel coding is always used to increase reliability at the expense of bandwidth, while gain in terms of transmitted power, which is specified as coding gain. The invention of turbo codes has moved the limit of achiev- 
able coding gain much closer to the Shannon limit [2]. Unfortunately, the iterative nature of the decoding algorithm for turbo codes leads to serious problems when it comes to efficient VLSI implementation. Each pass (iteration) through the data block involves large numbers of fetch/store, computation operation, and storage of large amounts of state information. Performing multiple iterations of an inherently serial process to achieve high coding gain (see Chapter 2) reduces throughput and increases power dissipation.

Compared to turbo codes, LDPC codes exhibit better decoding results due to their good distance property, reduced complexity and high parallelism. Unlike turbo codes, it is possible to decode LDPC codes using a block-parallel structure instead of a block-serial structure. Consequently, in order to obtain higher throughputs, there is great interest in implementing LDPC decoders of parallel architecture [7] [8] [10] [23]. More recently, it has been observed that iterative decoding algorithms on probability propagation networks may be well suited for analog VLSI [11] [12]. Analog iterative decoding circuits, which are power efficient, fast and physically small, have been demonstrated by some researchers in recent years [17] [18] [29] [23].

Although recent research activity on LDPC decoders has made significant progress in various directions, the conventional analog approach remains unsuitable for large-scale parallel analog LDPC decoder design, which may involve hundreds or even thousands of processing units with complex interconnection wiring. Due to the difficulty of VLSI implementation, especially in the case of analog VLSI, these designs are often limited to only a small subclass of codes, or even some specific code [13] [15]. In general, good LDPC codes are irregular codes with large block sizes. The randomness of the parity-check matrix and the large block size of the encoded data make analog implementation, 
which is usually done manually, quite time-consuming and prone to errors. Even for non-random matrices, this is a challenge. The performance of the fabricated chip is then usually adversely affected by less-than-ideal placement and routing. This crucial issue determines how well we can exploit the benefits of LDPC codes in real applications. Finding an optimization method for general LDPC code that can be integrated into an automatic analog design flow remains to be solved. This thesis addresses this challenge.

\subsection{Research Contributions}

This thesis is based on previous work [26] done by Hemati, a former $\mathrm{PhD}$ student and a member of our research group. He used a canonical analog design flow method to build a CMOS analog minsum iterative decoder for a $(32,8,10)$ LDPC code. The macro cells built by Hemati for his own work will be incorporated into the new optimization method.

The contributions of the present work are as follows:

1) This thesis shows how a digital placement and routing tool can be used to optimally implement a complex analog LDPC decoder design.

2) Our iterative optimization method is applied in three different cases. The results demonstrate a substantial improvement over our initial random placement.

3) To facilitate the automatic design process, a Matlab program was written, which automatically generates structured Verilog code appropriate to any kind of $H$ matrix. (This will make future work, which involves the design of additional library cells, much easier). The automatically generated Verilog code will be useable by the First Encounter (FE) placement and routing tool. 


\subsection{Organization of Thesis}

- First, to allow for a better understanding of the content of this thesis, Chapter 2 gives a background review of topics related to iterative decoding: LDPC codes, Tanner Graph representation, and iterative decoding algorithms.

- In Chapter 3, some issues related to VLSI implementation are discussed. After comparisons are made between implementation platforms (DSP, FPGA, ASIC), structures (parallel, serial) and approaches (digital, analog), a brief circuit description of macro cells (a degree-4 check node and a degree- 3 variable node) follows.

- Chapter 4 begins with a thorough discussion of optimization metrics, then presents a detailed description of the new iterative optimization method. Comparisons of optimal and ad_hoc results are made.

- Finally, the results and conclusions of this work are summarized in Chapter 5, along with suggestions for future research work. 


\section{Chapter 2 Background Review}

The goal of this chapter is to provide readers with a basic understanding of channel coding and the associated iterative decoding algorithms. The topics will include LDPC codes themselves, their TG representations, and iterative decoding algorithms.

\subsection{Channel Coding and Iterative Decoding}

Coding can be divided into two categories: source coding and channel coding. They are used in very different ways. Source coding compresses the source information by reducing redundancy (e.g. ZIP, JPEG, MPEG2), while channel coding reduces the bit error rate (BER) by adding redundancy during information transmission (e.g. LDPC codes, Turbo codes). Channel coding provides a means of patterning signals so as to increase reliability while reducing their energy consumption for a given error performance.

LDPC codes belong to the class of channel coding, which is used to detect errors caused by noise in a digital transmission system. Code evaluation is usually based on code rate (R), SNR $\left(E_{b} / N_{0}\right)$, and BER. An important metric used to evaluate code performance is coding gain, which is defined in terms of the saving in $E_{b} / N_{0}$ to achieve a given BER when coding is used vs. no coding. Generally, the lower the code rate, the higher the coding gain. Good LDPC codes are usually irregular codes with long block length and large girths. (Girth is defined as the length of the shortest cycle.) 
Figure 2.1 shows a digital transmission system consisting of a data source, a source encoder for compression, a channel encoder to add parity check bits, a transmitter, a noisy channel, a receiver, a channel decoder for error correction, a source decoder for expansion, and finally the data sink.

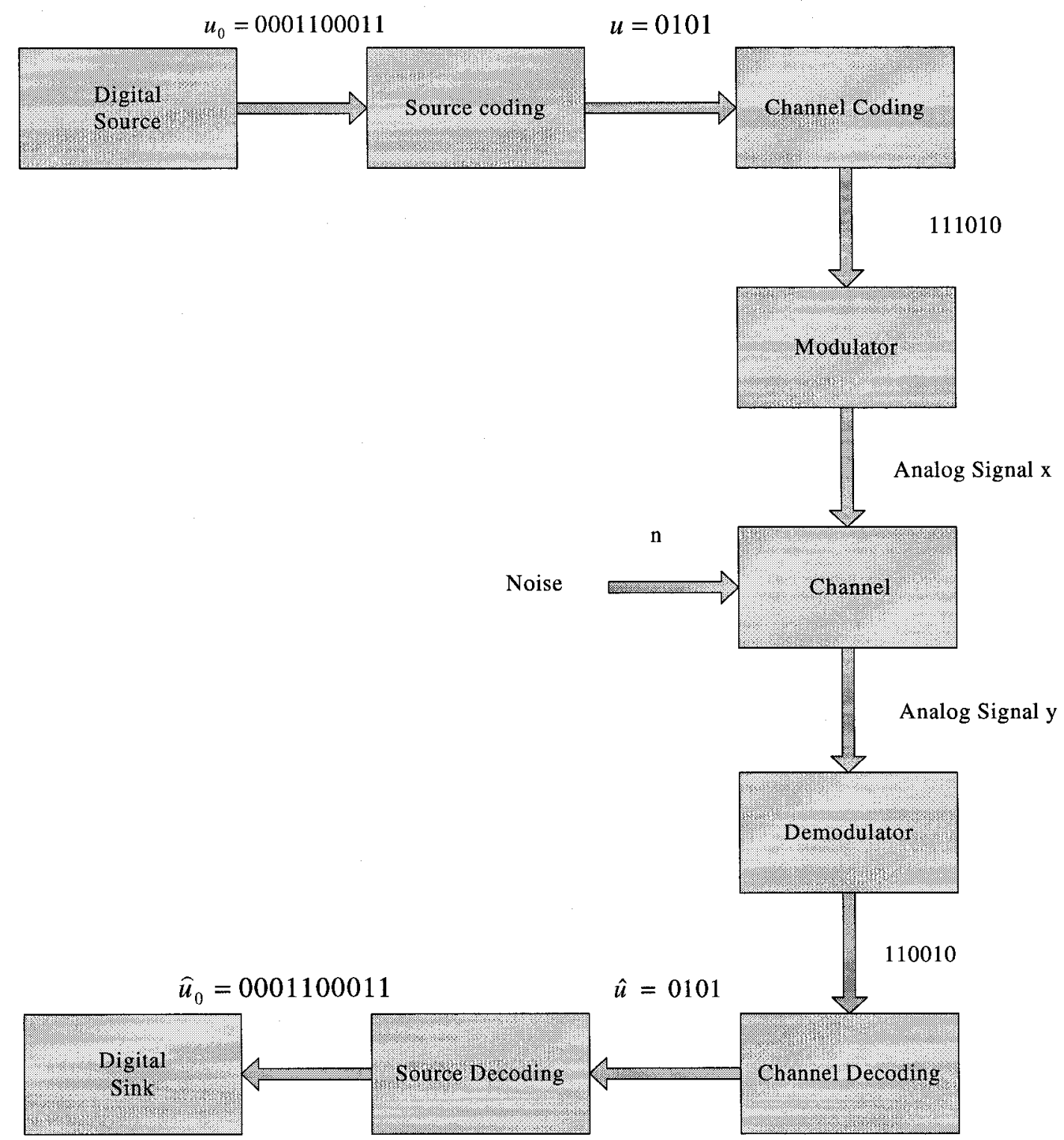

Figure 2.1 Simplified Classical Model of a Digital Communication System 
An iterative decoder is constructed from two or more soft information components (units that realize certain computation functions, e.g. add, multiply), which operate exclusively on probabilities for both their input and output. During decoding, each decoder component computes extrinsic information probabilities. "Extrinsic" here means that the output message sent along one edge does not depend on the incoming message sent along the same edge. The components then exchange this extrinsic information and decode again. Because of this information sharing, the decoder's calculation tends to improve after each decoding iteration.

\subsubsection{H Matrix \& LDPC Code}

LDPC codes are linear block codes defined by a sparse binary matrix $H$, called the parity check matrix. The set of all code words $\mathrm{C}$ can be fully described by its parity check matrix $H$ through equation $(2.1)$

$$
H x^{T}=0, \forall x \in C
$$

In general, LDPC codes can be non-binary. In this thesis, discussion will be limited to binary code only. The code word $x$ can be fully described by the code's parity check matrix $H$, which is a sparse binary matrix. That means most elements in this $H$ matrix are "0" rather than "1". In other words, the density of " 1 " in this LDPC code is low. The sets of row and column elements are chosen to satisfy a desired row and column weight profile, and maximize the length of cycles in the graph representation of the matrix for better coding gain. An example of an $H$ matrix is shown in Figure 2.2 below. 


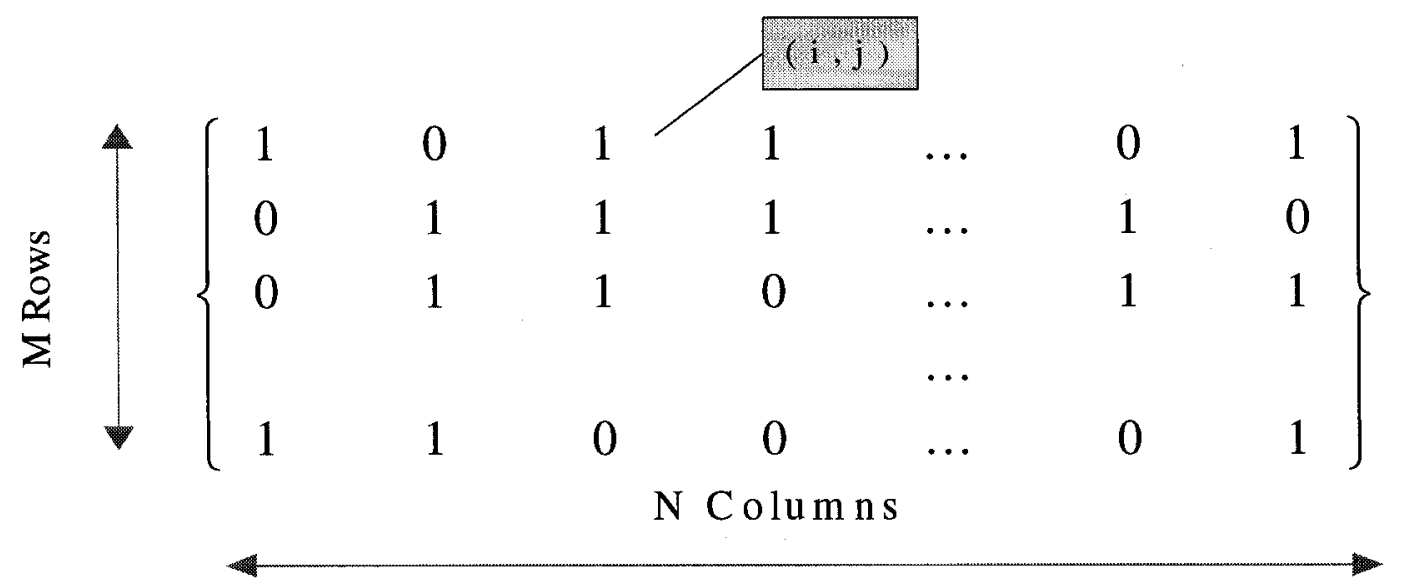

Figure 2.2 Sample of a Low-Density Parity Check Matrix $H$

Each column of $H$ is associated to a codeword bit, and each row of $H$ corresponds to a parity check. A non-zero element in the $(i, j)$ position indicates that data symbol $j$ participates in parity check $i$. If in a block of $\mathrm{N}$ bits or symbols there are $\mathrm{M}$ redundant parity symbols, the code rate $\mathrm{R}$ is given by:

$$
R=\frac{N-M}{N}
$$

An LDPC code is called "regular" when the parity check matrix has the same weight (number of " 1 "s) in each row and in each column. Otherwise, it is irregular. For regular LDPC code with an $M \times N$ parity check matrix, with weight of column $W_{c}$ and weight of row $W_{r}$, the density is

$$
\rho=\frac{W_{r} \times M}{M \times N}=\frac{W_{r}}{N}=\frac{W_{c} \times N}{M \times N}=\frac{W_{c}}{M}
$$

Gallager's [3] original codes are regular. A further study described in [5] shows that the performance of irregular LDPC codes is usually better than that of the regular ones. 


\subsubsection{Graph Representation (Tanner Graphs)}

Like any other linear block codes, an LDPC code can be fully described by a bipartite graph. The bipartite Tanner Graph (TG) contains only two types of nodes: symbol (bit) nodes (shown as circles in Figure 2.3), and check (constraint) nodes (shown as squares). A TG representation of a linear block code is shown in Figure 2.3.

A TG can be constructed based on the parity check matrix of the code. The symbol nodes correspond to the columns of the parity check matrix $H$, and the check nodes to the rows. A variable node $V_{j}$, corresponding to column $\mathrm{j}$ of the $H$ matrix, is connected to check node $C_{i}$, corresponding to row $\mathrm{i}$ in the $H$ matrix, if the entry $(\mathrm{i}, \mathrm{j})$ in $H$ is " 1 ".

\section{Check Nodes}

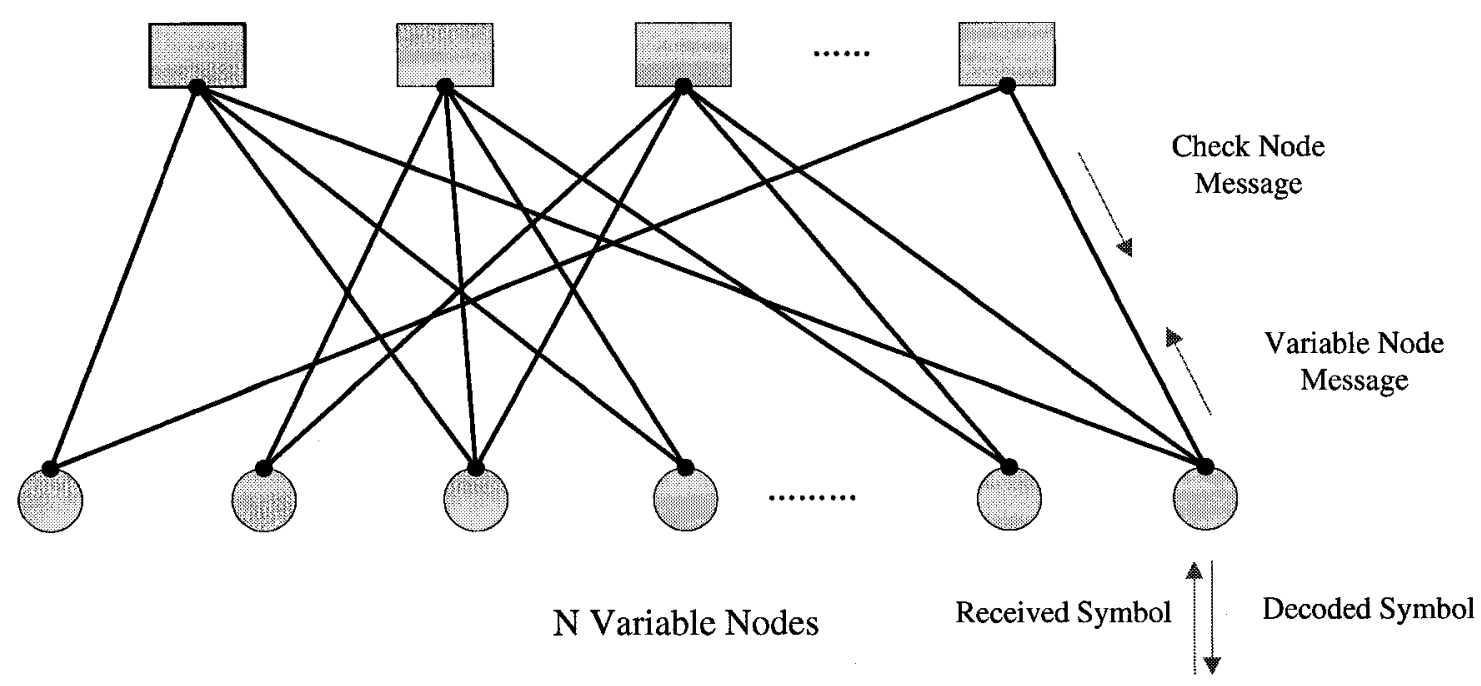

Figure 2.3 TG Representation of a LDPC Code with the $H$ Matrix from Figure 2.2 plus Information Flow in the Message-Passing Algorithm 


\subsubsection{Soft Decision Versus Hard Decision}

Traditionally, the first operation in the algebraic decoding of linear block codes is to determine whether the received information bit is a "1" or a " 0 ". An applicable algorithm, e.g. a parity-check equation, is used to extract the data word from the code word. The step of deciding between a " 1 " and a " 0 " is called a hard decision. However, with a hard decision, some information is lost. For example, no matter how close the information bit is to a " 1 ", it is considered a "1" as long as it has more distance to a " 0 ". Even if in situation $S 1$, the information bit is much closer to " 1 " than in situation S2, the two situations are treated as being the same and part of the information is lost. A way to avoid losing information is to use probability to indicate how close the information is to a " 1 " or " 0 ", then the probability of being a "1" in situation $S 1$ is much higher than in situation $S 2$. As a result, no information is lost. This is called a soft decision. A soft decision is superior to a hard decision with regard to error rate, but a more complex decoder is needed.

\subsection{Iterative Decoding Algorithms}

A number of iterative decoding algorithms can decode LDPC codes efficiently. Generally speaking, an iterative decoding algorithm starts by creating initial (local) messages (weights) for each symbol node using the observations at the output of the channel and the channel characteristics. It then passes these initial messages to the check nodes along the edges of the TG. The two-step iterations below then continue until a certain condition is met.

1) Iteration Step 1: Check nodes processing

Check nodes process the incoming information and pass new (extrinsic) messages to the symbol nodes. These messages usually reflect the estimate of the check nodes regarding the value and reliability of the symbol nodes. 
2) Iteration Step 2: Symbol nodes update

The symbol nodes receive incoming messages from the check nodes and send updated (extrinsic) information about their value and associated reliability back to the check nodes. The output messages are updated at the same time.

A hard decision on the value of each output bit (e.g., "0" or "1") will be made after each iteration. When certain situations are satisfied, the iteration will be terminated. These situations could include the following:

1) The outputs of the hard decision satisfy all the check equations; or

2) The maximum number of iterations has been reached.

The message computation and transmission can be carried out in different domains. For example, messages can be represented by their original form in the probabilities domain. They can be represented in the Likelihood Ratio (LR) domain, Log-Likelihood Ratio (LLR) domain, or Likelihood Difference (LD) domain [46].

There is a wide variety of iterative algorithms for LDPC codes. Among the various decoding algorithm variations, both belief propagation (BP [3], [35], also called sum-product algorithm) and the Min-sum (MS) [36] algorithm are frequently applied in the LDPC codes decoding process. 


\subsubsection{Belief Propagation (BP) in the LLR Domain}

$\mathrm{BP}$ is a generic algorithm that operates on a factor graph via a sequence of local computations at every factor-graph node. The computation rules consist only of multiplications and additions in probability domain. Hence BP is also called sum-product. With regard to BER, the decoding algorithm is optimal if the assumption there are no cycles in the graph holds. However, in many cases, the assumption is not valid. Due to the presence of cycles in the graph, the calculation is not optimal after a few iterations. There are techniques [37] [45] which are used to improve the performance of BP. In [37], a multiplicative factor and an additive factor have been used to reduce the absolute value of the outgoing LLR messages at variable nodes, respectively. In [45], a multistage iterative decoding algorithm that combines BP with ordered statistic decoding has been used. With these techniques, the modified BP algorithms perform very well.

There are two types of computational modules for VAR and CHK nodes that depend on the type of algorithm and domain in which they are implemented. For the BP algorithm in the LLR domain, the operation can be expressed as shown below:

Check node update in LLR domain [26]

$$
L_{i}=2 \tanh ^{-1}\left(\prod_{\forall i, j \neq i} \tanh \frac{L_{j}}{2}\right)
$$

Symbol Node Update in LLR domain [26]

$$
L_{i}=L_{i}^{(0)}+\sum_{\forall j, j \neq i} L_{j}[26]
$$


M Check Nodes

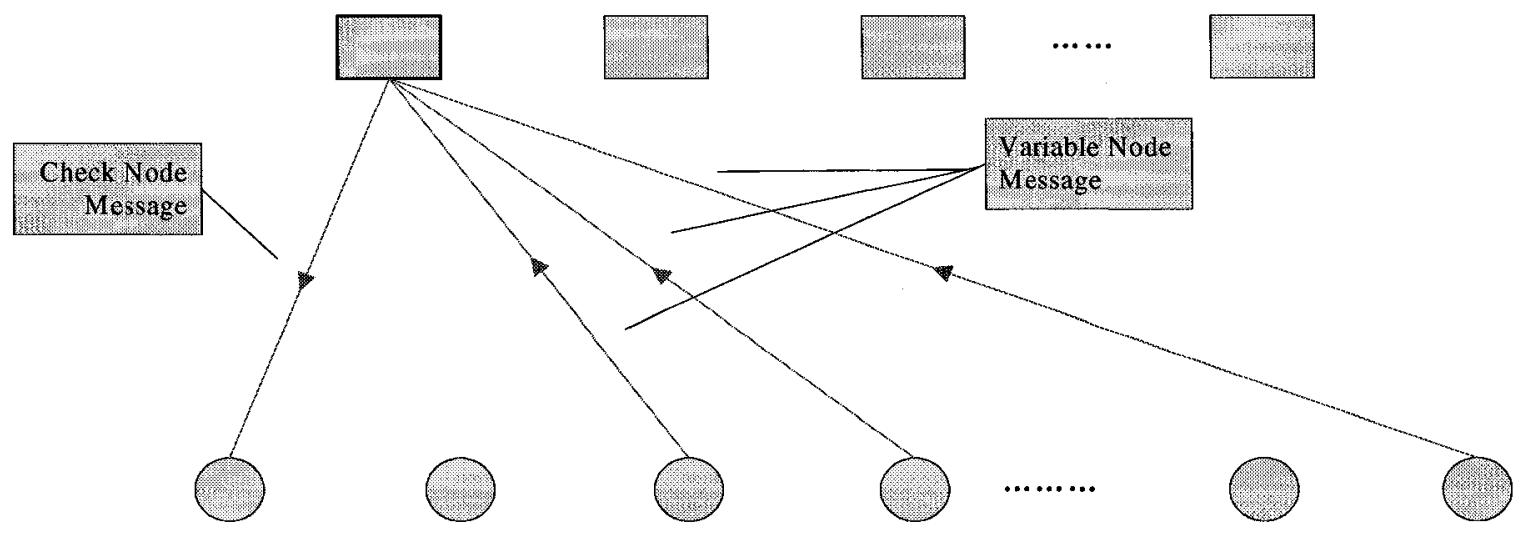

$\mathrm{N}$ Variable Nodes

Figure 2.4 Simplified Tanner Graph Showing Message-Passing Scheme for Check Node

The update taking place at a symbol node during the $q^{\text {th }}$ iteration is illustrated in Figure 2.5.

\section{Check Nodes}

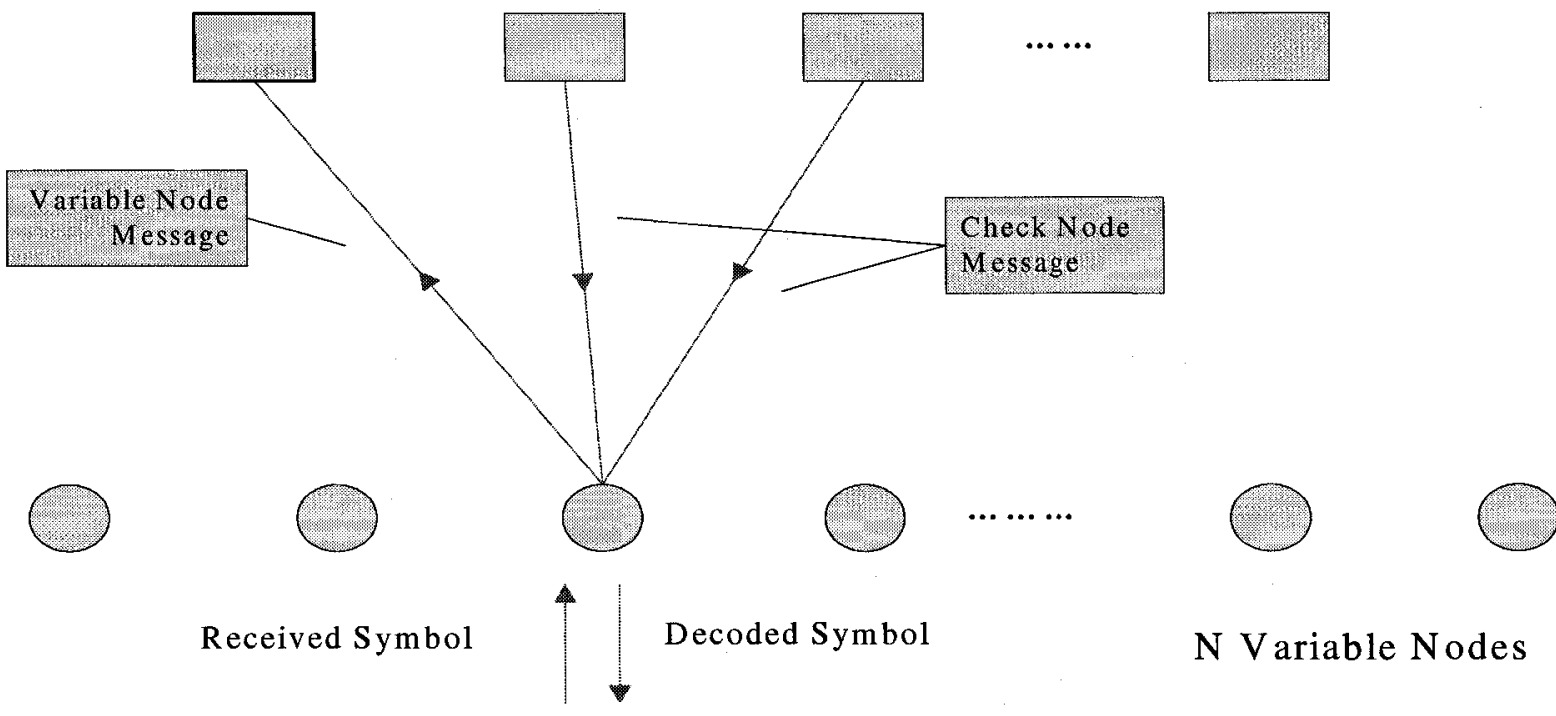

Figure 2.5 Simplified Tanner Graph Showing Message-Passing Scheme for Variable Node 
It is preferred that the actual computations of iterative decoding perform in the Log-Probability or Log-Likelihood Ratios (LLR) domain based on probability values. The reason is that in the Log domain, multipliers are replaced with adders. However, evaluating the sum in the Log-Probability domain requires a combination of exponential and logarithmic functions. In order to simplify the implementation, the computation can be approximated with min-sum to compromise the decoding performance of the code to some degree.

\subsubsection{Min-Sum (MS) Algorithm in the LLR Domain}

The Min-sum algorithm [36], [38] can be deducted from Sum-product. The operation in check node can be expressed as

$$
L_{i}=\min _{\forall i, j \neq i}\left|L_{j}\right| \prod_{\forall i, j \neq i} \operatorname{sign}\left(L_{j}\right)
$$

The information update in the variable node is the same as BP:

$$
L_{i}=L_{i}^{(0)}+\sum_{\forall j, j \neq i} L_{j}
$$

Where $L_{i}^{(0)}$ is the initial local message at variable node I, function $\operatorname{sgn}(\cdot)$ returns 1 or -1 depending on whether its argument is positive or negative. The initial message $L_{i}^{(0)}$ is computed by replacing the conditional probability density functions of the channel output corresponding to variable node i. It can be shown that for the binary input AWGN channel, $L_{i}^{(0)}$ can, in fact, be simplified to the received value $y$. Note that independence of the observations is assumed, which means that channel is memoryless. Also note we assume the factor graph is cycle-free. This is not satisfied in many applications, but the algorithm performs well if the cycles are long enough. 
As mentioned previously, the Min-sum (MS) algorithm can be regarded as an approximation of BP [36]. Other common names for the Min-sum algorithm are "max-sum" and "max-product". Although the performance of Min-sum is slightly (up to $0.5 \mathrm{~dB}$ for rate 0.5 codes and $0.2 \mathrm{~dB}$ for rate 0.9 ) worse than that of SP, the simpler implementations of the $\min ($.$) and sign(.) functions largely reduce the$ complexity of the check node calculations, and so the circuitry is less complicated and the chip area is smaller. Moreover, simple modifications to the MS can improve its error performance so it is very close to that of the BP algorithm [39]. Researches indicate that MS is more robust in the presence of quantization noise than BP [39]. The noise performance of large analog integrated circuits often suffers because of the mismatch problems stemming from fabrication imperfections. The high immunity of MS is therefore a very important consideration.

Both hard- and soft-decision forms of the message-passing algorithm may be used for iterative decoding, although the performance of soft decision is better than the other.

At each iteration, the hard decision assignment for each bit $v$ is decided based on

$$
\begin{aligned}
& L_{i}=L_{i}^{(0)}+\sum_{\forall j, j \neq i} L_{j} \\
& \hat{v}=\frac{1-\operatorname{sgn}\left(L_{i}\right)}{2}
\end{aligned}
$$




\section{Chapter 3 VLSI Implementation of LDPC Decoders}

In this chapter, important aspects of decoder implementation will be introduced: VLSI technology, the decoder architecture, and a brief introduction to the circuit construction of the analog decoder.

\subsection{LDPC Decoder VLSI Implementation}

The invention of the transistor at Bell Lab in 1947 revolutionized computation and signal processing. These small, inexpensive, low-power active devices soon replaced vacuum tubes in many applications. The electronics industry has achieved phenomenal growth over the last few decades since then, mainly due to rapid advances in integration technologies and large-scale systems design. Most subsequent development in these areas has taken place in the digital realm, with analog work being confined to some specific areas, such as analog-to-digital (ADC) and digital-to-analog (DAC) converters that convert naturally analog signals into bits and vice versa.

Designers prefer digital circuits to analog for several reasons. In digital circuits, a transistor can be simply modeled by a switch that is either open or closed, and designers are less concerned about the dynamics of the transistors. Digital circuits can be standardized and reused by other designers. In addition, digital circuits are more robust than analog circuits, and their performance will not deteriorate easily due to outside interferences or common fabrication-induced problems, such as transistor mismatch. However, this does not imply that for any signal processing application, the digital approach results in an efficient solution. In the case of a massively parallel algorithm, such as the artificial neural network demonstrated by Mead $[11,12]$, the analog circuit shows an advantage. In our case, for a 
large parallel LDPC code decoder, as will be discussed later, the analog approach actually has an advantage over its digital counterpart.

\subsubsection{Review of Different Implementations of Iterative Decoding}

The three implementation platforms for digital LDPC decoder designs are described in the following paragraphs. Digital Signal Process (DSP), Field Programmable Gate Array (FPGA) and Applicationspecified Integrated Circuit (ASIC) -based realizations have been chosen for the study and descriptive information and performance figures of some typical examples are listed in the first three entries of Table 3.1. (The last two entries in this table are related to analog design and have been placed in the same table to facilitate comparison.)

The performance characteristics that most greatly influence the choice of platform are high throughput, low power consumption, small chip area and short transmission latency. Also of considerable importance, but somewhat more difficult to quantify, are flexibility and scalability. The flexibility of a platform represents the ease, with which implementation can be updated for changes in the target specification. Scalability captures the ease of using the same platform for extensions to the application that may require higher throughput, increased code block size and a higher edge degree for LDPC codes.

The available platforms are ASIC, FPGA and DSP. DSP has a limited number of single-instructionper-cycle execution units but provides the most flexibility. Compared with an FPGA-based design 
and an ASIC design, the time required for a design cycle is short. The drawback is that performance is relatively poor. Performing a simulation with BER below $10^{-6}$ is a lengthy process. This platform naturally implements the serial architecture for iterative decoding.

Unlike DSP, design using an FPGA offers more opportunities for parallelism with reduced flexibility. Generally speaking, an FPGA is suitable for data path-intensive designs because its interconnected grid is optimized for local routing. However, the disorganized nature of a parity-check matrix and complex internal interconnections place a requirement that is hard to achieve on a significantly longer global routing. Existing implementations of LDPC decoders on FPGA continue to circumvent this problem by using time-shared hardware and memories in place of interconnections. This serial method currently limits the internal throughput of an LDPC decoder.

An ASIC-based LDPC decoder has impressive performance statistics, but these tend to come at the expense of device cost. The main contributors to this problem are the cost of the design itself, and the manufacturing cost. Today's ASIC design flow typically involves three stages, with a separate design team for each. The first team is responsible for the system-level design. Once it has finished, the second, register-transfer-level (RTL) coding team takes over. After this step is completed, the last team does the actual physical design. This process is time-consuming and expensive and the highly specialized design will lack the desirable characteristic of flexibility, which was defined earlier.

Compared with DSP and FPGA, a custom ASIC design is well suited to a direct mapped architecture, offering even higher performance with further reduction in flexibility. During the design, designers have the flexibility of using all available techniques to improve performance. An LDPC decoder implemented in 0.16 um CMOS technology achieves $1 \mathrm{~Gb} / \mathrm{s}$ throughput [8] by fully exploiting the paral- 
lelism in the LDPC decoding algorithm. The drawback of ASIC design is usually that logic density is limited to only $50 \%$ to accommodate large on-chip interconnections for a parallel architecture. In the case of a parallel architecture, the problem of scalability also remains. In addition, routing congestion arises when considering the implementation of high-performance LDPC code. For a code decoding with $0.1 \mathrm{~dB}$ of the capacity bound, a block size of tens of thousands is required. With 10 times more interconnection wiring, a parallel implementation will face imminent routing congestion and a huge routing area penalty. As wire length increases, $\mathrm{RC}$ delay increases accordingly. Critical route delay may become a new problem.

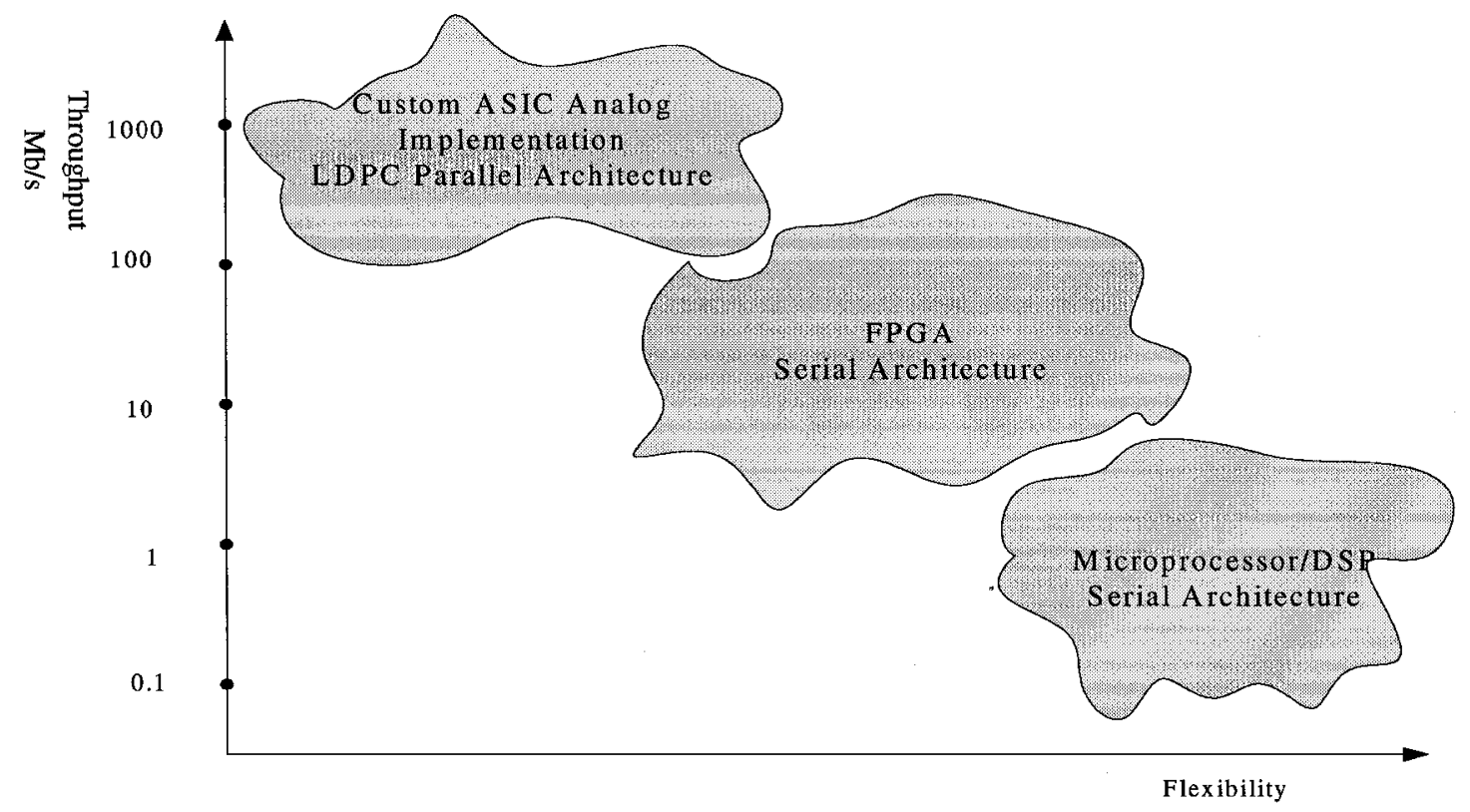

Figure 3.1 Relative Comparisons Among Different Platforms for LDPC Decoder Implementation

\subsubsection{Iterative Decoder Structure}

The order of message computations and their exchange distinguishes two main classes of decoders: parallel and serial. The parallel decoder architecture directly maps the nodes of a factor graph onto the processing elements, and the edges of the graph onto an interconnecting network. The parallel computation of messages requires the same number of processing elements as the number of nodes in the 
factor graph. On the other hand, the serial decoder architecture distributes the arithmetic requirements sequentially among a small number of processing elements. Due to the randomness and complexity of the $H$ matrix, the main challenge in VLSI implementation is how to effectively manage messagepassing during the iterative decoding process. The structure of an LDPC graph directly affects the implementation of the message-passing network. A graph with disorganized connections will either lead to routing congestion in parallel architectures or address indexing issues in serial architectures. However, there are samples of LDPC codes with highly structured and regular graphs [9] that can be exploited to allow the use of fast, small shift registers. Another technique used is column splitting on these structured codes. It also yields added parallelism between memory accesses in serial architectures with a limited number of parallel processing elements. Generally, the physical structure of the LDPC decoder falls into two categories:

1) Fully parallel decoders that directly instantiate the bipartite graph of the LDPC codes to the hardware. While the check node and the variable node are mapped to corresponding message computation function units, the edges are mapped to the interconnecting network.

2) Partially parallel/serial decoders that serialize and distribute an inherently parallel algorithm amongst a number of processing units.

A parallel realization of any algorithm will frequently be both throughput- and power-efficient, at the expense of increased area and routing congestion. On the other hand, serial realizations require fewer arithmetic units, and use memory elements in place of complex interconnections. 


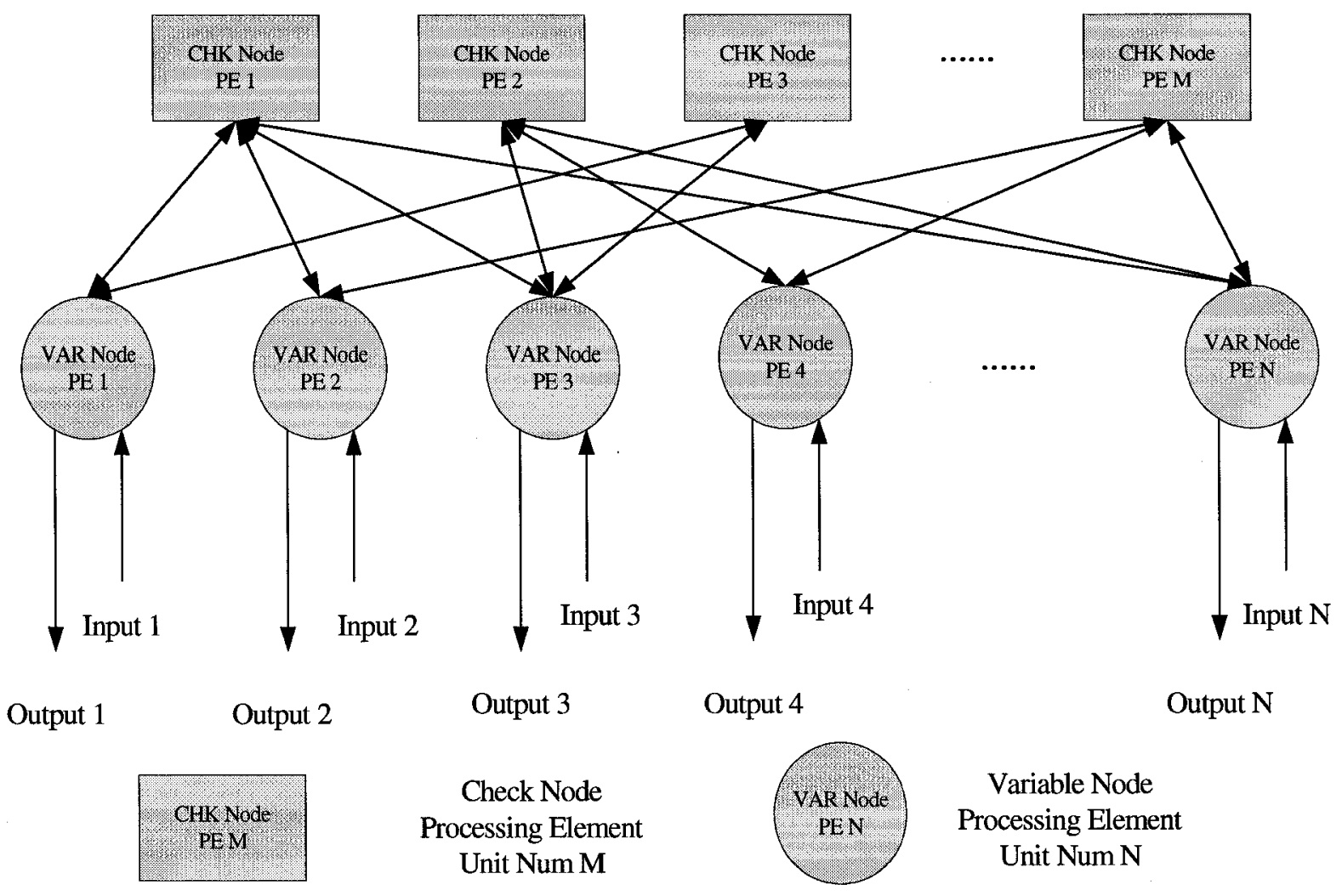

Figure 3.2 Full Parallel Structure of LDPC Decoder 


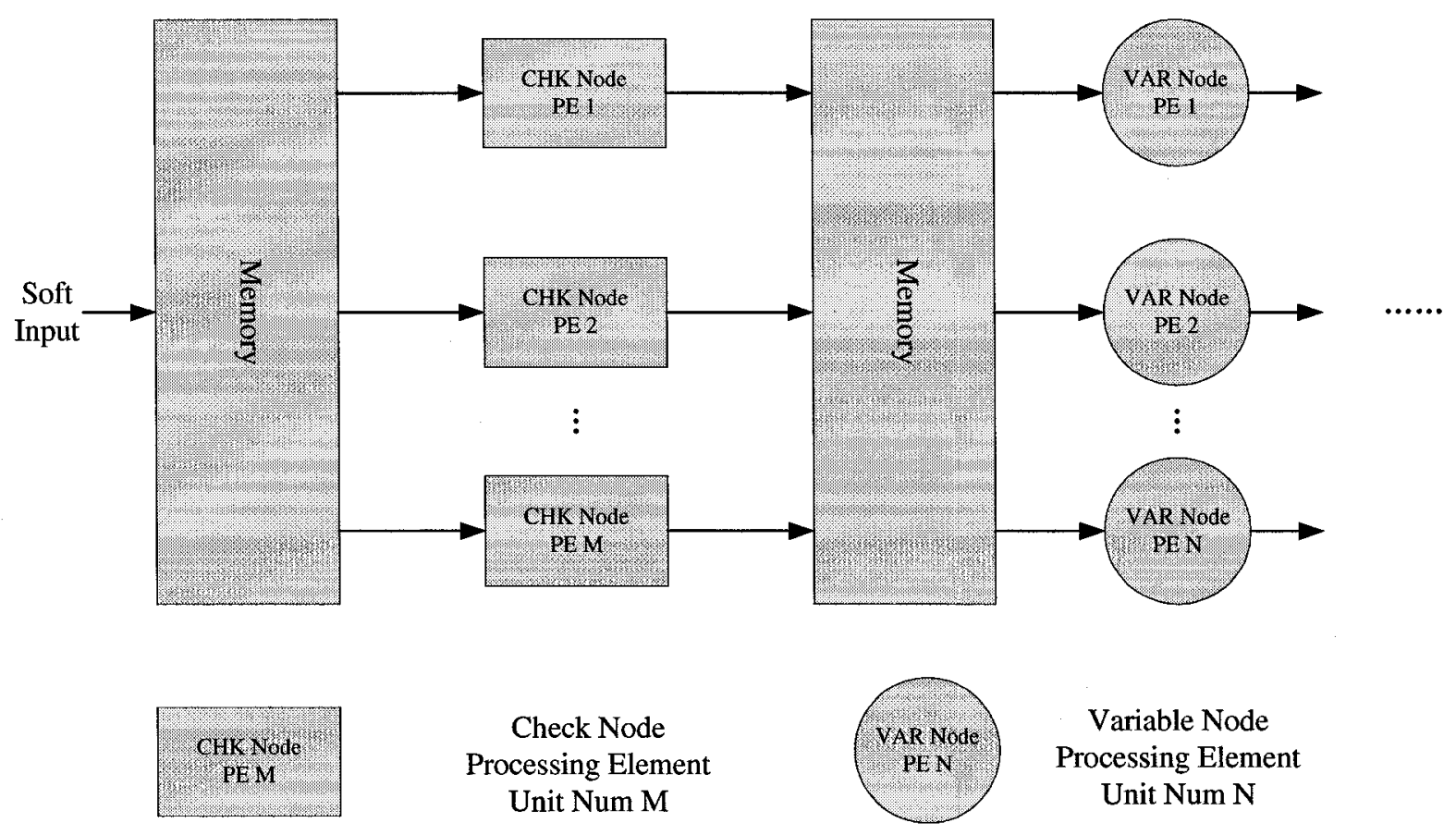

Figure 3.3 Serial Structure of LDPC Decoder

\begin{tabular}{|c|c|c|c|c|}
\hline Platform & Architec & cture & Speed & Implementation (chip area, transistor number, power) \\
\hline \begin{tabular}{l|l}
1 & $\mathrm{DSP}[27]$
\end{tabular} & Serial & Digital & $133 \mathrm{~kb} / \mathrm{s}$ & \\
\hline 2 FPGA[28] & Serial & Digital & $56 \mathrm{Mb} / \mathrm{s}$ rate & \\
\hline 3) Custom ASIC[8] & Parallel & Digital & $1 \mathrm{~Gb} / \mathrm{s}$ rate & $5.25 \mathrm{E}+07 \mathrm{um}^{\wedge} 2,1750 \mathrm{k}$ gates, $1.38 \mathrm{~nJ} / \mathrm{b}($ core $)$ or $690 \mathrm{mw}$ \\
\hline 4 Custom ASIC[29] & Parallel & Analog & $3.7 \mathrm{Mbp} / \mathrm{s}$ & $2.75 \mathrm{E}+04 \mathrm{um}^{\wedge} 2,1976$ transistors, $0.218 \mathrm{nj} / \mathrm{b}($ analog) \\
\hline 5 Custom ASIC[30] & Parallel & Analog & $80 \mathrm{Mb} / \mathrm{s}$ & $5.6 \mathrm{E}+05 \mathrm{um}^{\wedge} 2,18,800$ transistors, $5 \mathrm{~mW}(\max )$ \\
\hline
\end{tabular}

\begin{tabular}{|lll|}
\hline LDPC CODE & Technology & Implementation difficulty \\
\hline 1 rate 1/2 LDPC decoder & Fixed-point DSP, TMS320C6201 & Limited number of ALU \\
29216-b, rate1/2, $(3,6)$ regular & Xilinx virtex-E XCV2600E & Control for memory access \\
3 1024-b, rate 1/2, irregular code & $0.16 u m$ CMOS 5-LM & Routing congestion; not scalable \\
$4(8,4,4)$ hamming code & 0.18 um BiCMOS process,6-LM & Circuit for low voltage design \\
$5(32,8,10)$ LDPC Code & 0.18 um CMOS process 6-LM & Routing congestion; not scalable \\
\hline
\end{tabular}

Table 3.1 A Comparison of Different Platforms 


\subsubsection{Analog Approach for Iterative Decoding}

Digitization has entered every aspect of life; analog circuits have gradually been replaced by their digital counterparts. The idea of not immediately adopting a knee-jerk digital approach to a communications problem may be strange these days. The problems customarily associated with analog signal processing come to mind-the sensitivity to component variations, susceptibility to noise and power supply disturbances, temperature dependency, production-line test difficulties, poor scalability, and so on. But no law of nature says that digital is always best. C.A. Mead, analogue signal processing pioneer, demonstrated [11] [12] that it is possible to build analog signal processing systems that not only share the robustness of digital systems but also outperform digital systems by several orders of magnitude in terms of speed and power consumption. This analogue approach is characterized by exploiting, rather than fighting, the fundamental non-linearity of transistor physics by system design in order to achieve accuracy at system level despite imprecise local subsystems and components [26].

In the case of analog LDPC decoder design, a very important but also understated advantage is that the number of interconnections can be greatly reduced from that encountered in a conventional digital approach (the problem of interconnection congestion mentioned earlier). The complexity of wiring is a great hurdle in the VLSI implementation of an LDPC decoder. Fortunately, the parity-check matrix for LDPC code is sparse by definition and the number of edges is only a few times larger than the block length. For the digital approach, even for an LDPC code with a sparse $H$ matrix of short block length, the physical interconnections are prohibitively large because the information exchanged is represented by number of bits in an analog circuit rather than "1". Usually four to six bits represent a piece of information and three bits is the minimum requirement in the digital approach. In addition, every piece of information is exchanged in a bilateral direction. As an example, in [8], for a (1024, 
512) irregular LDPC code that has 3,328 edges in its Tanner Graph, each message is represented by four bits. Consequently, the total physical interconnections will be

$$
3328 \times 4 \times 2=26624
$$

The physical consequences of such a high number of interconnections are first that there is a huge capacitive load on the active devices, and also that a substantial fraction of the chip area (50\% of 52 $\mathrm{mm}^{2}$ in this case with $0.16 \mathrm{~m} 5$ layers metal CMOS technology) is taken up by essentially nonfunctional elements. With regard to the huge physical interconnections saving, the analog approach could be preferable because every message can be represented by a current or a voltage in a wire in single-ended signaling. The amplitude of the current or voltage represents the value in an analog circuit, as opposed to the four to six bits required for a digital implementation. For differential signaling (when the message is represented by the difference between two voltages or currencies), an extra wire should be added. An extra saving in interconnection wire length also stems from the difference in design methods between the analog and digital approach. In analog design, the process for breaking down the decoder into processing elements according to functions and interconnections means the cells design could be a local optimization process in circuit performance, area and interconnecting wire length. In contrast to analog design, a synthesis tool breaks down the behavior or functional units into small logical standard cells without taking the physical connections in digital design flow into consideration. This synthesis process increases the complexity of the interconnections, although during the breakdown process there could be stages that involve different function units. During the placement, these small logical cells with interconnections are distributed around the allocated placing area rather than side-by-side according interconnection relations. For analog design flow, the saving in interconnection wires, especially those long global routing, means an improvement in routability, less routing congestion, and a smaller routing area, which could occupy up to $50 \%$ of the total area. 
Another reason that an analog design may be preferred over a digital design is power consumption. Considering that the voltage/current swing in an analog circuit is much less than its digital counterpart, the power consumption in each interconnection could be smaller.

An analog decoder can operate product function in the probability domain, evaluating the sumproduct algorithm in its original form. The following equation holds for a working bipolar transistor:

$$
I_{c}=I_{s}\left(1+\frac{V_{C E}}{V_{A}}\right) e^{\frac{V_{B E}}{V_{T h}}}
$$

Where $I_{c}$ is the collector current, $I_{s}$ is a constant current with typical values between $10^{-16}$ and $10^{-14}$ A. $V_{C E}$ is the collector-emitter voltage, $V_{A}$ is the early voltage with a typical value between 15 and $100 \mathrm{~V}$, and $V_{t h}$ is the thermal voltage and is equal to $26 \mathrm{mV}$ at room temperature. Probabilities, represented as currents in bipolar junction transistors, are multiplied using a six-transistor Gilbert cell [21] that takes advantage of the exponential relationship between collector current and base emitter voltage.

Similarly, an analog decoder can be implemented with MOSFET working in sub threshold state $\left(V_{g s}\right.$ is small). The following formula holds if source and substrate are short-circuited and $V_{D S}$ is large enough.

$$
I_{D}=I_{o} \frac{W}{L} e^{k\left(\frac{V_{G S}}{V_{T h}}\right)}
$$


This thesis focuses on the optimization of an analog decoder with a pre-built cell library rather than how the circuits are built to realize a certain function. A detailed explanation of how circuits are built is beyond the scope of this work.

Many problems have to be solved before analog LDPC decoders can be applied in the real world. Currently, analog decoders only exist in fully parallel architectures with small block sizes. For a serial architecture with a larger block size, a track-and-hold circuit can be employed.

\subsection{Design of Analog Macro Cells}

In this section, we will briefly introduce two macro cells (a degree-4 check node and a degree-3 variable node), which will be used in our analog automatic design flow for a parallel structure LDPC decoder. Again this thesis is not concerned with the detailed circuit. The detailed circuits, which are implemented using standard six-metal-layer, 0.18um CMOS technology, can be found in [26]. For better understanding, in this chapter, Figures 3.4, 3.5, and 3.6 come from [26].

To facilitate future work and to smooth the macro cells build-up process, a structured design approach is preferred. Several basic function blocks were built according to the functions of the different processing units. These blocks are listed below. 
For the variable node:

n-type current mirror, p-type current mirror. Both current mirrors include two versions: one for high swing current and the other for high impedance.

For the check node:

RTAS, ASTR, Min, XOR

By carefully designing these basic cells, a local optimization in circuit performance, chip area and interconnection wire length can be achieved. This should receive greater emphasis since local optimization in wire connections is naturally lost in the digital approach. It is a very common situation in digital design that the small standard cells are distributed in an area rather than placed together according to the interconnection. This is partly due to the synthesis and lack of optimization during the placement process.

\subsubsection{Variable Node Architecture}

In the variable node, incoming messages (extrinsic information) are added to the information (intrinsic information) received from the channel to generate the variable node's outgoing messages. Messages are represented in the form of currents and can be either positive or negative. Since the message or current can be either positive or negative, we need both types of current mirrors that can either draw current or inject current. The combination of both current mirrors in a circuit is called a current buffer. 


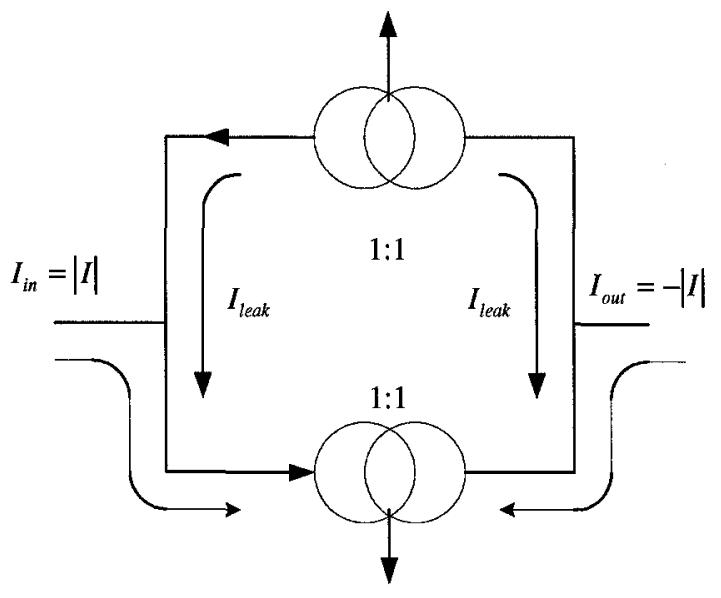

(1)

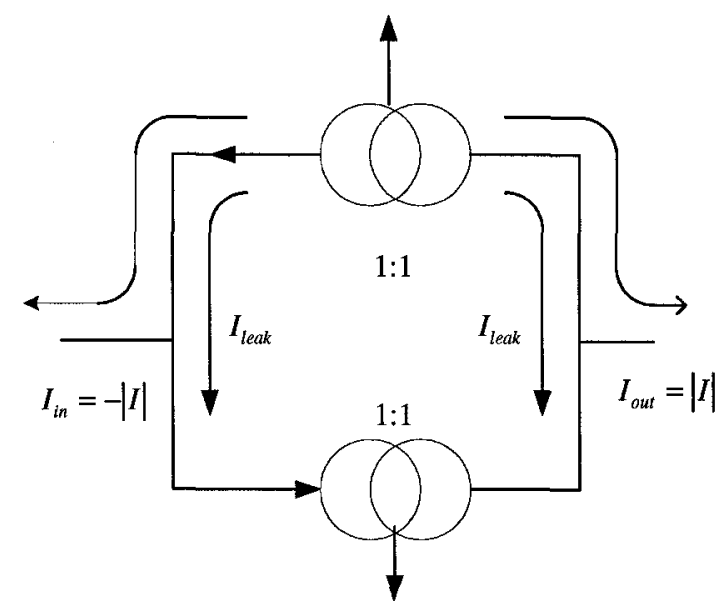

(2)

Figure 3.4 Current buffer with (1) n-type current mirror active

(2) p-type current mirror active

We will use the following example to show the structure of a degree- 3 variable node. The detailed circuit can be found in [26]. In our case (degree-3 variable node), according to $\left(L_{i}=L_{i}^{(0)}+\sum_{\forall j, j \neq i} L_{j}\right)$, the message transfer between variable nodes can be

$$
\begin{aligned}
& L_{V_{1} \rightarrow C_{1}}=L_{V_{1}}^{(0)}+L_{C_{2} \rightarrow V_{1}}+L_{C_{3} \rightarrow V_{1}} \\
& L_{V_{1} \rightarrow C_{2}}=L_{V_{1}}^{(0)}+L_{C_{1} \rightarrow V_{1}}+L_{C_{3} \rightarrow V_{1}} \\
& L_{V_{1} \rightarrow C_{3}}=L_{V_{1}}^{(0)}+L_{C_{1} \rightarrow V_{1}}+L_{C_{2} \rightarrow V_{1}}
\end{aligned}
$$

The output will be

$$
L_{V_{1}}=L_{V_{1}}^{(0)}+L_{C_{1} \rightarrow V_{1}}+L_{C_{2} \rightarrow V_{1}}+L_{C_{3} \rightarrow V_{1}}
$$

Based on the formulas $(3.4,3.5,3.6$, and 3.7) above, the circuit for Formula 3.4 can be built as 


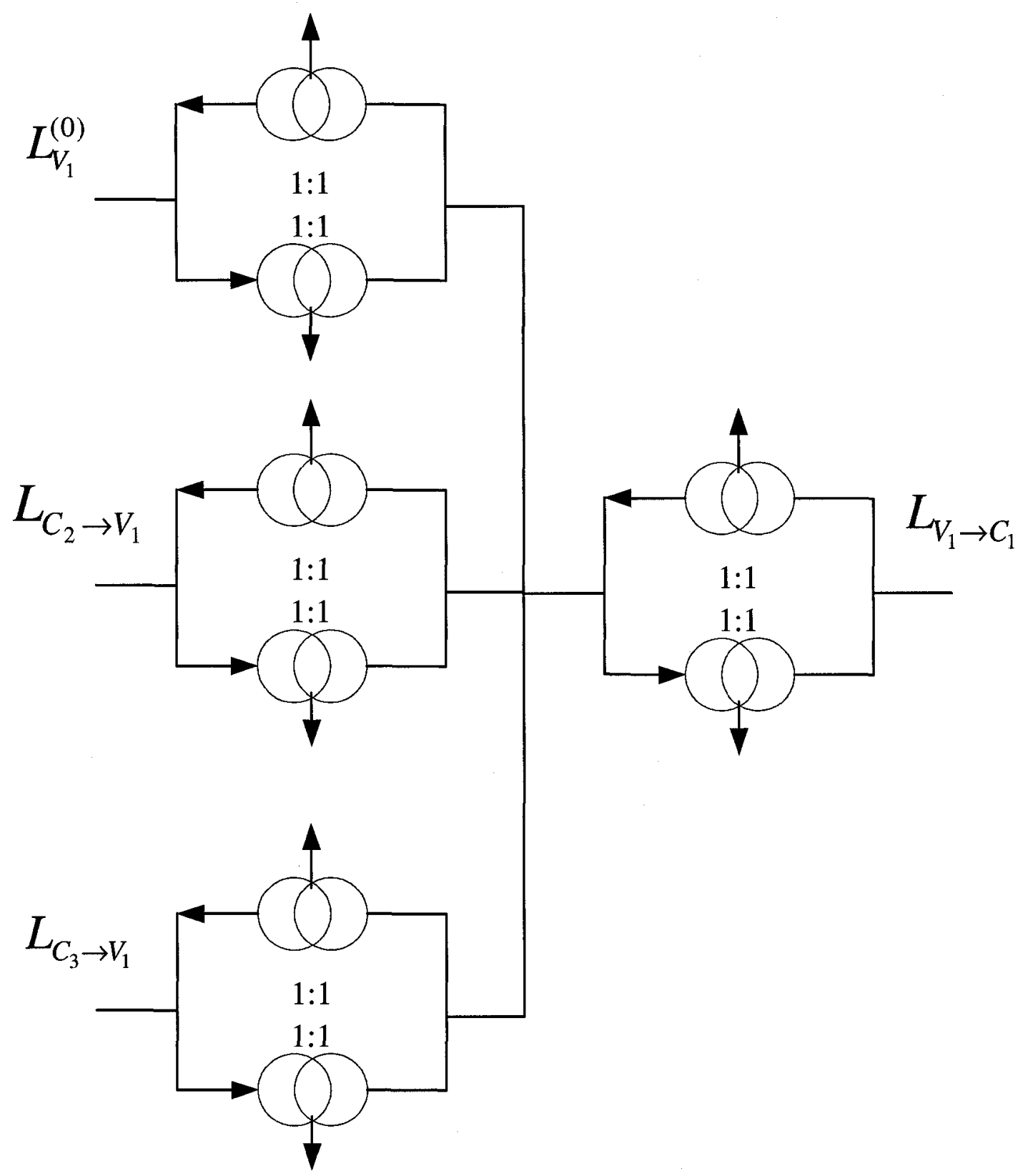

Figure 3.5 Partial Circuit of the Variable Node for Formula 3.4

The circuits for the other equations can be built in the same way to implement variable macro cells. 


\subsubsection{Check Node Architecture}

In this section, only the basic structure of parity-check nodes is presented, for better understanding. For detailed circuits, please refer to [26].

The operation in the check node is given by $\left(L_{i}=\underset{\substack{\forall i, j \neq i \\ \forall i n}}{\min , j \neq i}\left|L_{j}\right| \prod_{j} \operatorname{sign}\left(L_{j}\right)\right)$. Figure 3.6 shows the structure of a check node. For all incoming messages from the variable nodes, the RTAS model separates the sign and the magnitude messages. The magnitude messages are sent to the min-WTA model and the sign messages are sent to the sign model, which performs a XOR function. Then the minimum magnitude for the corresponding variable node is picked out by the min-WTA model and sent out to its destination (the ASTR model). At the same time, the sign values are calculated by a XOR model and sent to the ASTR model. The ASTR model combines the received magnitude with the sign message and sends it back to the corresponding Variable node. 


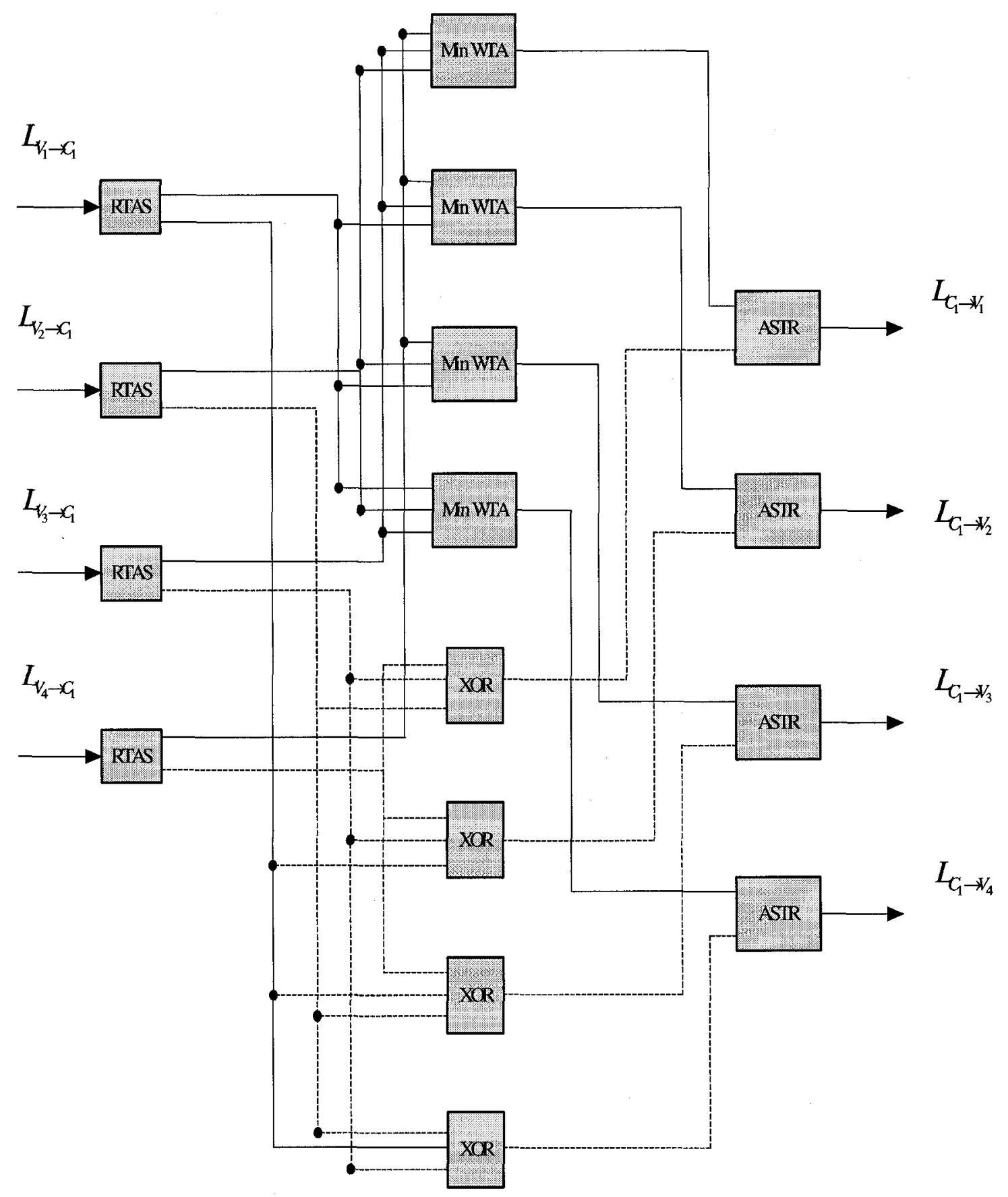

Figure 3.6 Basic Structure of a Degree-4 Check Node 


\section{Chapter 4 Iterative Optimization Method}

Research by Mead [11] [12] shows an analog circuit can outperform its digital counterpart in some areas e.g. computation power [48]. His idea is applied to analog decoding. The research by other scholars in related areas show that the LDPC decoder may be better suited for analog VLSI than its digital counterpart. Meanwhile, many questions remain unanswered, including how to optimize the complex interconnection wires in order to have higher throughput, less power consumption, with less routing area. The research [26] [47] shows that the throughput of a decoder is dependent on the average time constant of interconnections rather than the distribution of delay among the computational models. Based on this conclusion, a poor optimization result (total interconnection wire length) leads not only to poor throughput, but also greater economic expense with regard to chip area. The solution should not only achieve a satisfactory result (higher throughput, smaller chip area, lower power consumption) compared to the canonical design flow, but also smooth out the design process, in terms of reducing the difficulties (caused by the complex interconnections) encountered by a designer as well as helping to reduce errors (caused by the complex interconnections too) while achieving better results. In analog LDPC decoder design, interconnections are too complex for human beings to manually draw even for small, regular LDPC codes with a short block size, e.g. less than one hundred bits.

Although automatic VLSI implementation for large analog circuits is far from satisfactory, the digital process is rather mature. Various CAD tools dedicated to digital design are available on the market, but there are no CAD tools to optimize interconnection wires for analog automatic design flow. One of the main reasons for this lack of automation is that analog design in general is perceived as less systematic and more heuristic than digital design. The popularity of digital circuits also limits the 
need for analog CAD tools. Even the mixed signal design is a relatively new concept, despite the fact that the analog circuit has a longer history than its digital counterpart. Fortunately, there are placement and routing tools like First Encounter $(\mathrm{FE})$, which do not differentiate between digital and ana$\log$ circuits in some situations. As long as a netlist, which could be a structured Verilog code, correctly describes the structure of the circuit, and the homemade analog cells library meets the requirements, it can handle these analog cells the same way as in digital design. 

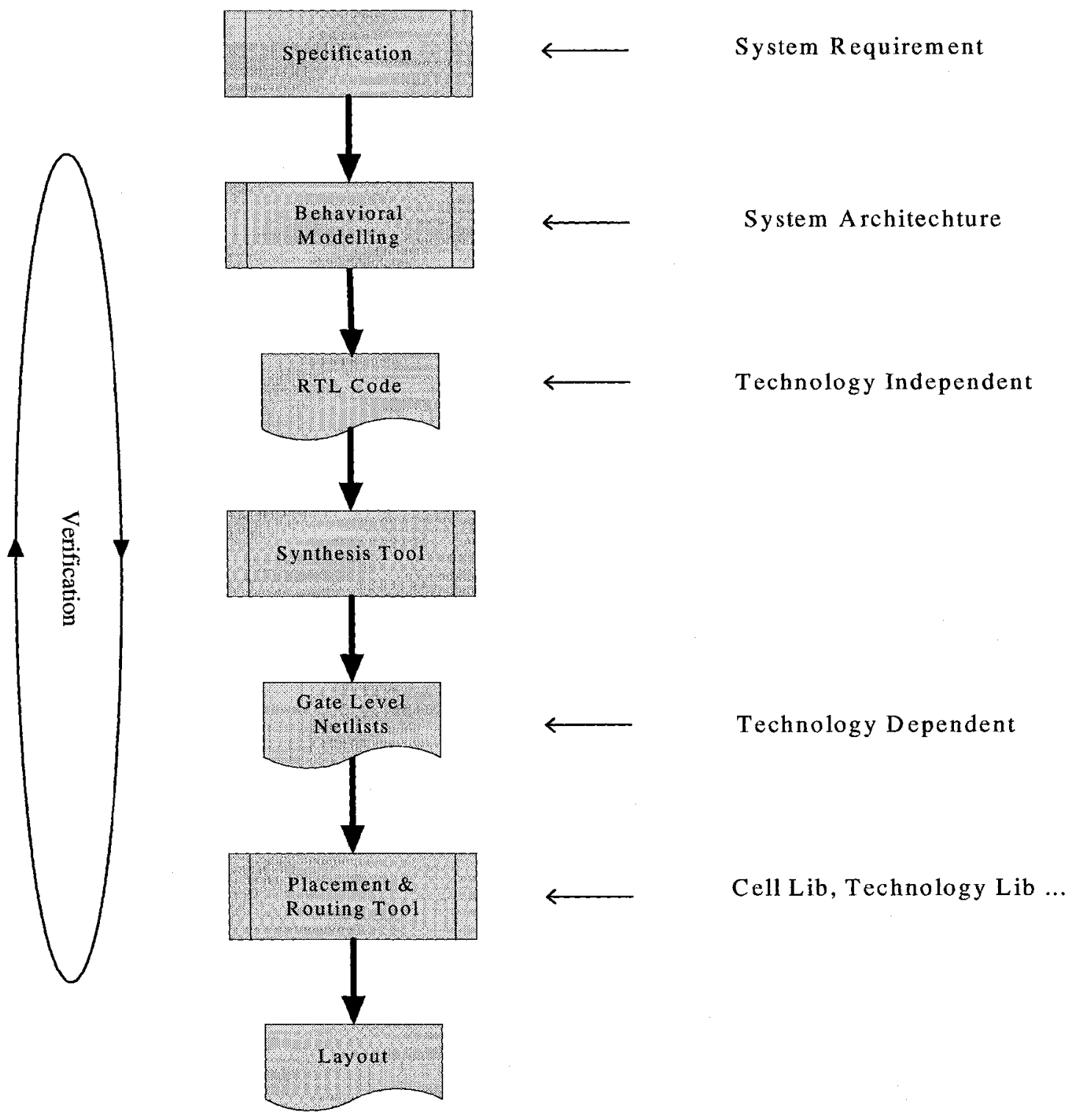

Figure 4.1 Traditional Digital Design Flow 


\subsection{Current Optimization Methods for an Interconnecting Network and Our De- sign Flow}

\section{Current Optimization Methods}

With the increasing demand for high-throughput decoders, the complexity of the hardware implementation of LDPC decoders has been gaining attention. In the published implementation of a practical LDPC decoder hardware [8], Blanskby et al. describe a digital high-throughput LDPC decoder implemented with parallel structure. They report that routing congestion is the determining factor in the area of the chip (50\% utilization). Furthermore, they also mention that because of the large number of global nets, most of the power dissipation is a result of the switching activity of the wires. Because of the probelems mentioned above, there is plenty of room for improvement.

Due to the issues mentioned above, code design has become a promising direction for research, as the interconnecting network is mapped directly from the $H$ matrix. In order to make codes practical, it is important to consider the hardware implementation-related issues during LDPC code design. The primary restricting factor of most known parallel implementations in LDPC decoders is the complexity of the graph connectivity associated with random-like LDPC codes. The complexity of decoders is linear in their code length. The problems arise from the fact that LDPC codes of random/regular structure require large block sizes for good error correction performance, leading to prohibitively large chip sizes. These extra considerations restrict the choice of good codes, which are usually irregular, with large block sizes. In order to produce a viable real-time iterative decoder, a lot of work has been done in the area of code generation in an attempt to reduce the interconnection complexity [15] [31] [32]. In order to aggressively exploit the power and throughput advantages of parallel archi- 
tectures, techniques in code construction that address the complexity of routing in parallel structure implementations have been developed. Current approaches to handling hardware-related issues have been limited to generating LDPC codes from special families of graphs (e.g., Ramanujan graphs) [13] [14], which impose constraints on the code parameters. Another approach uses simulated annealing [15] to generate LDPC graphs satisfying certain conditions. Campeliot et al. describe the bit-filling [16] method to generate LDPC graphs, given constraints on the degree of the nodes and girth (length of the smallest cycle in bipartite graph). For many reasons, codes suitable for use may have to trade SNR performance for improved partitioning properties that would assist with the routing problem.

All the optimization methods mentioned above focus on code design rather than a universal optimization method, which can be applied to implement any code without restrictions. A close watch on the traditional design flow also hints that an implementation is usually far from being optimized, whether it is an analog design or digital design. In analog design, the placement and routing are based on a designer's intuition and experience rather than any systematic way to achieve a reliable optimized result. How to choose an optimized location for a specific macro cell is a problem to be solved.

\section{Our Improved Design Flow}

In designing an analog LDPC decoder of parallel structure, drawing schematics and layouts of analog decoding networks becomes too complicated for large codes. An analog system of up to a few hundred transistors can be drawn very easily if a structural design approach is used. But imagine a large factor graph of several thousands of individual nodes with different degrees. How can one make sure that, after a long day of drawing interconnection lines between the individual building blocks, mistakes are not made with the drawing? It is certainly better not to have to rely on one's own drawing 
capability if a schematic description file can be generated by a computer program. Additionally, the design time may take far too long, even if a human can handle the complicated schematic, and changes in the design may require a complete redrawing of all the elements of the network. Hence we have to look for a design flow that offers an automatic construction of the decoding network. The aim is thus a computer-assisted methodology that checks the critical elements, i.e. the interconnections in the schematic and in the layout of the circuit chip. In the context of coding, the structure of a block code is described by its parity check matrix $H$. This matrix may serve as the basis for many different design steps: The code's performance can be evaluated using the matrix in a simulation program, but it may also serve as the basis for a schematic generation program. In general, it will not be possible to design a large analog network first-time-right without computer aided design.

Researchers [17] in this area propose an automatic design flow for large-scale analog LDPC decoder design. They note that LDPC codes have a limited number of degrees for check nodes and variable nodes. At the same time, the complete circuit can be built on the foundation of a limited number of basic cells. It is not surprising that researchers attempt to build up a basic analog cells library, which instantiates the digital design approach to realize an automatic design flow. For different situations, this method has to be dramatically modified from case to case at a certain stage. Figure 4.2 explains an automatic large-scale analog LDPC design flow, which is used in [18].

The approach of [17] can reduce the complexity encountered by a designer while designing a LDPC decoder with a large block size. It is shown in [17] that analog error control decoders can be built using a small number of basic cells in a cell library, facilitating automatic synthesis. Moreover, their analysis shows that analog error control decoders built using that method are comparable in perform- 
ance, with smaller size and lower power than the corresponding canonical designs. But at the same time, the interconnection wires are still too complex and they do not have an optimization method that can be integrated into their design flow. Another problem is that although breaking down the variable node and check node to some basic cells helps to automate the design process, it increases the complexity of interconnections compared to traditional analog design. This method makes the unsolved interconnections optimization problem even more complicated. 


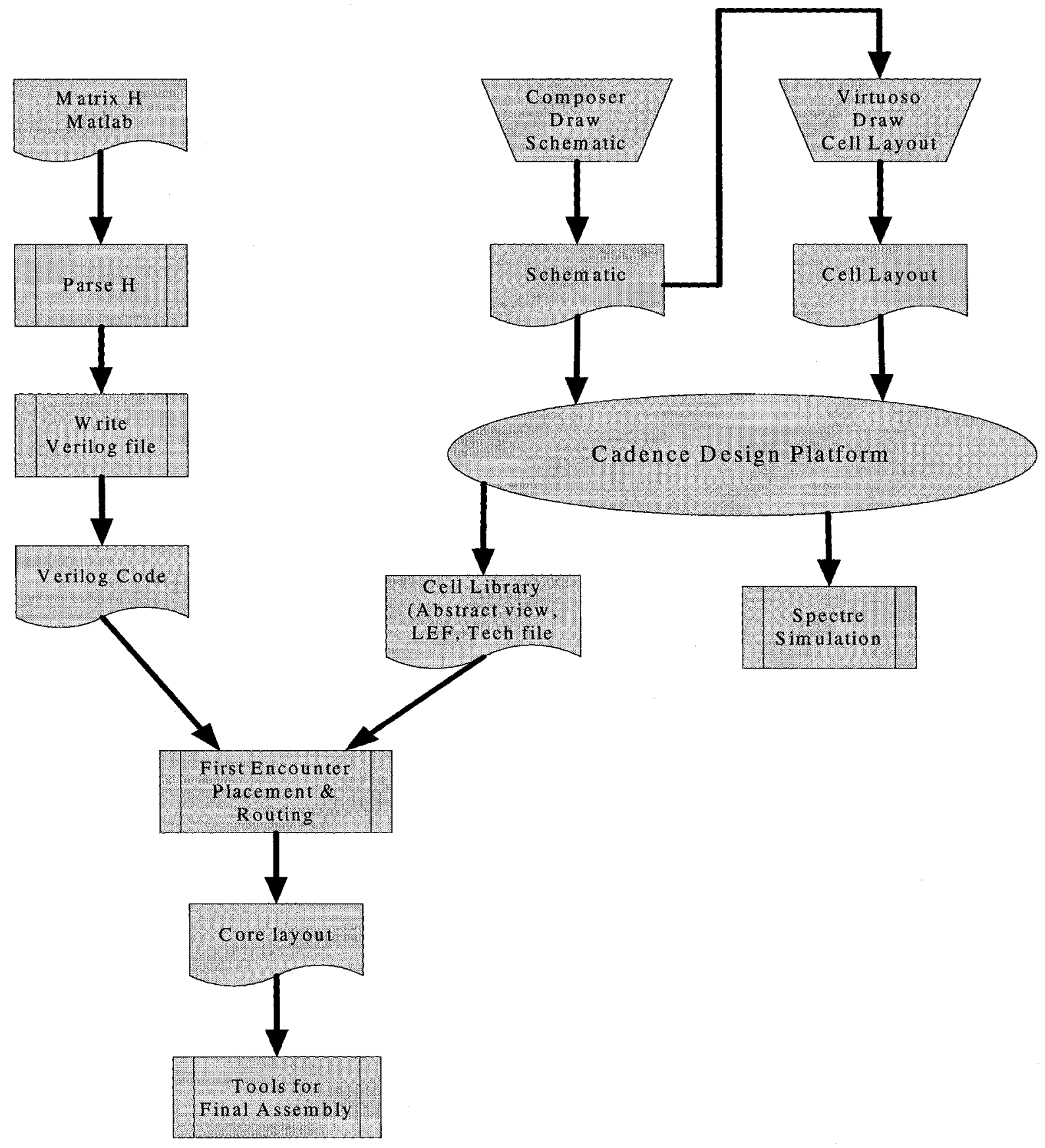

Figure 4.2 Overview of Design Flow [18] Used to Automate the Construction of a Large-Scale LDPC Analog Decoder 
In our situation, the research is based on a cell library that includes basic function cells and two prebuilt macro cells. Those cells are introduced in Chapter 3. With this pre-built cell library, an analog decoder [23] [26] [34] for $(32,8,10)$ LDPC code was completed successfully. Our design flow, incorporating interconnection, will be designed in such a way that it can reuse the key parts (pre-built cells) of the previous design, and furthermore has flexibility for future expansion, e.g. the cell library could include check nodes and variable nodes for other degrees as well as an irregular parity check matrix.

Refer to Figure (4.3), a design flow integrated with our placement optimization method is proposed. This optimization method can be applied to shorten the total interconnection wiring using a pre-built cell library with flexibility in a parity check matrix (there is no restriction on the parity check matrix). The cells used in the placement and routing are the check node processing unit and variable node processing unit. The iterative optimization process starts from an initial placement. For the initial placement, there are no restrictions regarding where a specific cell is placed, as long as this cell is placed according to the floor plan. After the iterative optimization process starts, the tendency is to place global connecting nodes (nodes have long connecting wires) at a relatively optimized position to realize optimization at a global connection level. The local optimizations (cells optimization), which include the check node, variable node, RTAS, ASTR, etc., are realized during the cell build-up process, which is a traditional analog design flow. The localized optimization helps to reduce the total complexity of the interconnecting network while the global optimization is realized by the iterative optimization method. 


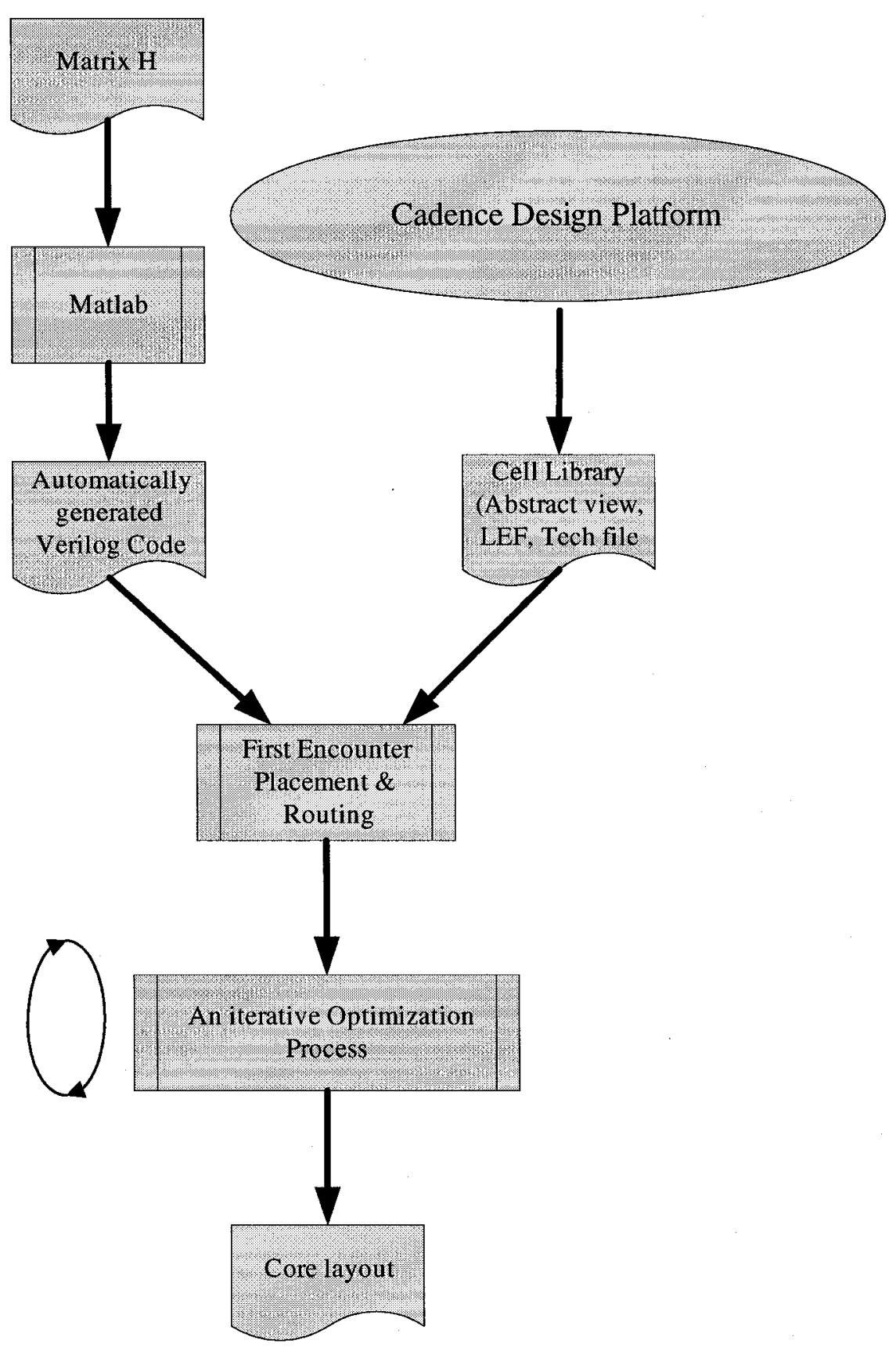

Figure 4.3 Design Flow Used to Automate the Construction of a Large Analog Decoder Suitable for an Iterative Optimization Process 


\subsection{Optimization Metrics}

The process of placement and routing is actually a process of optimization using an inbuilt optimization engine. Since the advent of chemical-mechanical polishing (CMP) in the mid-1990s, which allowed more than three layers, area routers have largely replaced channel routers. Area routers make several passes over a design, using simple heuristics to route nets. On each pass, wires are created that may conflict with each other. After routing, the number of conflicts is determined. The largest set of wires with no conflicts is kept, while the other wires are ripped up and rerouted on the next pass. For this reason, area routers are sometimes called "rip-and-replace" routers. This process continues until all nets are routed, with no conflicts. Area routers are able to achieve a much denser layout than channel routers when four or more metal layers are available. Cadence's placement and routing tool, First Encounter (FE), has been selected for use in the new design flow.

Closely related to routing, cell placement is a fundamental problem in VLSI computer aided designs (CAD), which is also a very important part of our design process. Objects such as wire length and area minimization have long been major concerns. With the advent of a deep sub-micron design, the scope of the research on placement optimization has now expanded to include interconnection delay optimization, power minimization, signal integrity, routability, etc. In our iterative optimization method, we focus on finding an optimized position for a cell while assuming that certain situations (configuration for layout, relative position of variable and check node) are a given. 
Before an optimization method is proposed, the metric used to evaluate the effect of optimization should be established. Independent research emphasizing different aspects of iterative analog decoder have adopted various evaluation metrics to measure optimization result. These measurements can be based on chip area [13] [26], power consumption [6] [24] [29], interconnection wire length [26], or chip speed [24] [26] [33], or evaluate all these parameters in a comprehensive manner. Below, we will first discuss the properties of interconnection wires in CMOS technology, then establish the relationships among the most important parameters (throughput, power consumption, as well as chip area).

\section{The Capacitance of Interconnection Wires}

Since all the interconnections in our case are between macro cells, we assume all the connections are metal rather than poly. A picture of capacitive coupling is shown in Figure 4.4.

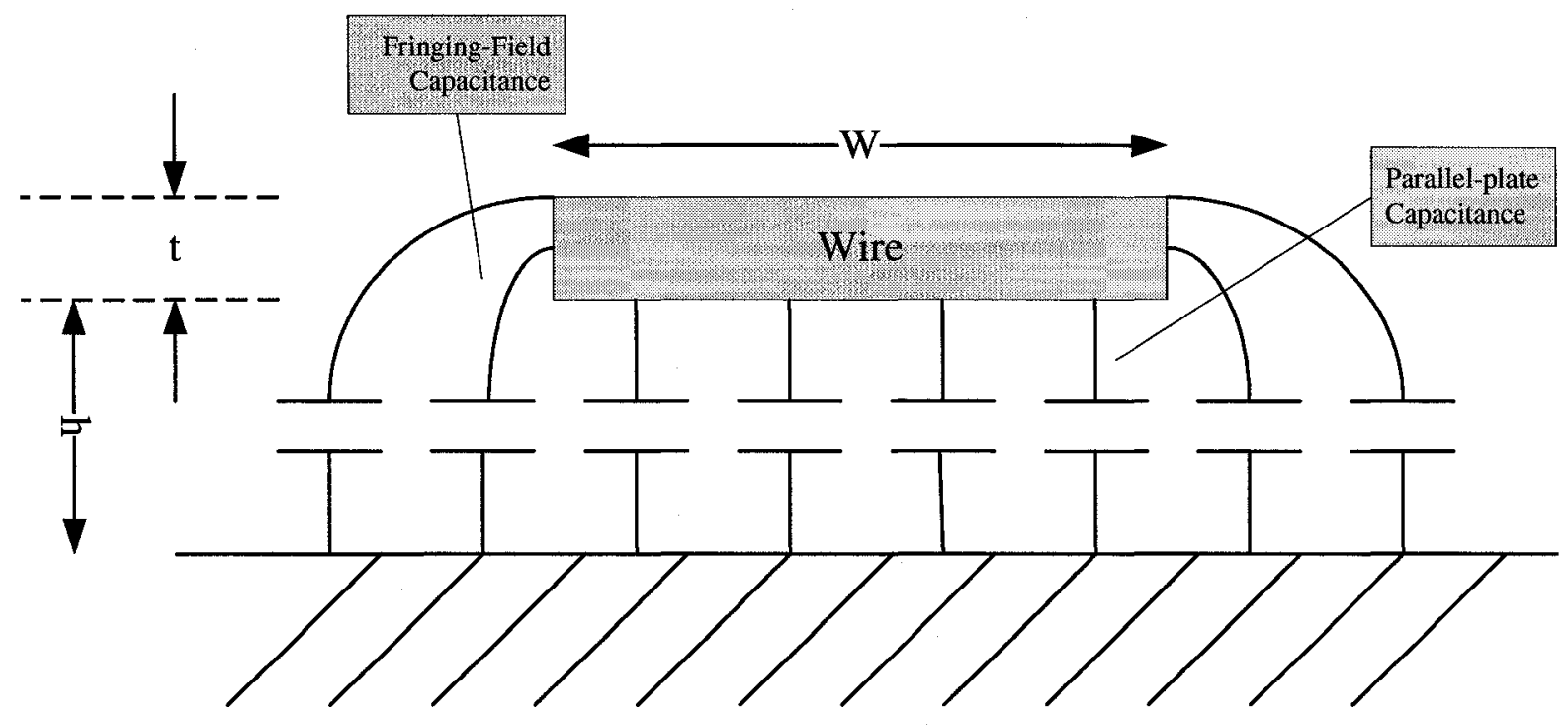

Substrate

Figure 4.4 Capacitive Coupling Components 
In a large-scale integrated circuit, the parasitic interconnection capacitances are among the most difficult parameters to estimate accurately. Each interconnection line (wire) is a three-dimensional structure in metal with significant variations in shape, thickness, and vertical distance from the ground plane (substrate). A simple parasitic capacitance per unit area can be approximated by

$$
c=\frac{\varepsilon_{0} \varepsilon_{r} A}{h}
$$

where $\mathrm{A}$ is the area of the trace and $h$ is the distance from the substrate. $\varepsilon_{0}=8.85 \times 10^{-12} \mathrm{~F} / \mathrm{m}$, $\varepsilon_{r}=3.9$ for $\mathrm{SiO}_{2}$. For a long line, the capacitance per unit length, taking the fringing capacitance into account, can be determined by [20] [21]

$$
c=\varepsilon_{0} \varepsilon_{r}\left[\frac{W}{h}+0.77+1.06\left(\frac{W}{h}\right)^{1 / 4}+1.06\left(\frac{t}{h}\right)^{1 / 2}\right]
$$

Then the total capacitance will be $l \times c$, which shows that the total capacitance will increase linearly with the length.

\section{Resistance of Interconnection Wires}

For low frequency signals, the resistance of a rectangular conductor in an integrated circuit is given by

$$
R_{\text {wire }}=\frac{\rho l}{A}=\frac{\rho l}{w t}=R_{\text {sheet }}\left(\frac{l}{w}\right)
$$

where $\rho$ is the resistivity of the conductor material, $l$ is the length of the conductor, and $R_{\text {sheet }}$ represents the sheet resistivity of the metal wire. 
For higher frequency signals, the skin-depth effect will begin to occur. When a conductor carries a high frequency signal, it tends to conduct only around the periphery of its cross-sectional geometry. Because less of the conductor is used to conduct the signal, this results in an increase in the resistance of the interconnections. The skin-depth effect could result in signal distortion as well as degradation of the circuit performance. The resistance of a conductor experiencing skin-depth is given by

$$
R_{\text {wire }}=r(f) l=\frac{l \sqrt{\pi f \mu \rho}}{2(t+w)}
$$

where $f$ is the frequency and $\mu$ is the permeability of the metal. As can be seen from both equations (4.3) and (4.4), the resistance of an interconnection wire is linearly dependent on the interconnection wire length both for low frequency and high frequency.

\section{Power Consumption}

Since the optimization is based on pre-built macro cells, we will only consider the power consumption outside the macro cells. The total power dissipated in charging and discharging a wire with capacitance $\mathrm{C}$ is given by Equation (4.5)

$$
P=\frac{1}{2} f_{e f f} C V^{2}=\frac{1}{2} f_{e f f} c l V^{2}
$$

$f_{\text {eff }}$ is the activity factor denoting how many times the voltage on the wire will swing in one second. $c$ is the capacitance per unit length, $V$ is the amplitude of voltage in the wire and $l$ is the total interconnection wire length. From equation (4.5), power consumption depends linearly on the length of the wire, which makes $l$ a good indicator for the power consumption of an interconnection wire. 
Energy efficiency, which is the ratio of average power and throughput, is commonly used to evaluate the performance of a decoder. Assuming the power consumption of macro cells is optimized, a shorter total wire length will lead to an improvement in energy efficiency since less power will be lost in interconnections.

\section{Interconnection Delay}

As with power consumption, we care only about the interconnection delay between macro cells. For a simple lumped RC delay model, assuming that the capacitance is discharged initially, and the rising output voltage reaches the 50\%-point at $t=\tau$; thus we have [21]

$$
\tau \approx 0.69 R C=0.69 r c l^{2}
$$

where $r$ is the resistance per unit length, while $c$ is the capacitance per unit length. $l$ is the interconnection wire length. As the wire length for a specific interconnection wire is optimized to be shorter, the total wire length will decrease accordingly, which means the total wire length can be a proper valuation metric for the optimization effect.

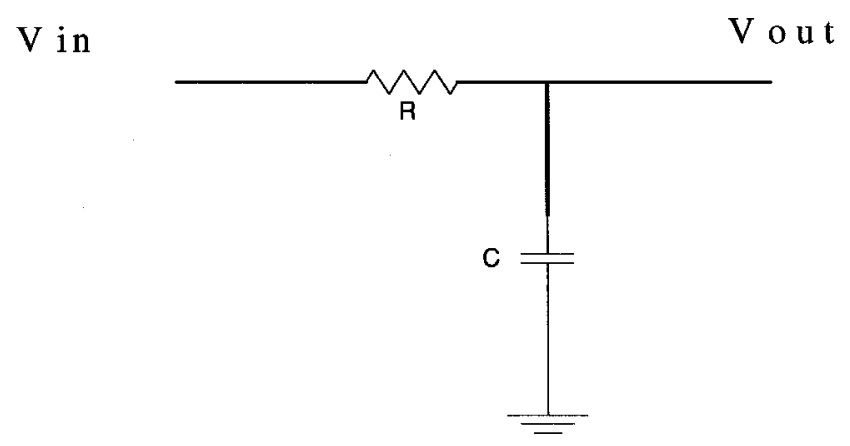

Figure 4.5 Simple Lumped RC Model of an Interconnection Wire

( $\mathrm{R}$ and $\mathrm{C}$ represent the total line resistance and capacitance, respectively.) 
This simple lumped RC network model provides a rough approximation, showing that the delay is proportional to the square of the wire length. Again, from equation (4.6), the interconnection wire length $l$ can be a good evaluation of interconnection delay. Unfortunately, this simple lumped RC network model provides a very rough approximation of the actual transient behavior of the interconnection line. A more accurate representation using a RC ladder network is shown in Figure 4.6 below. With a very large $\mathrm{N}$ (distributed RC line behavior), the RC delay can be simplified to

$$
\tau=\frac{R C}{2}=\frac{l^{2} r c}{2} \quad \text { for } N \rightarrow \infty
$$

A detailed discussion on this RC model [20] is beyond the scope of this thesis.

The result is smaller than a simple lumped RC model. In both models, since R and C are linearly dependent on the interconnection wire length between macro cells, an optimized total wire length will eventually lead to a reduced RC delay.

Total N

Segments

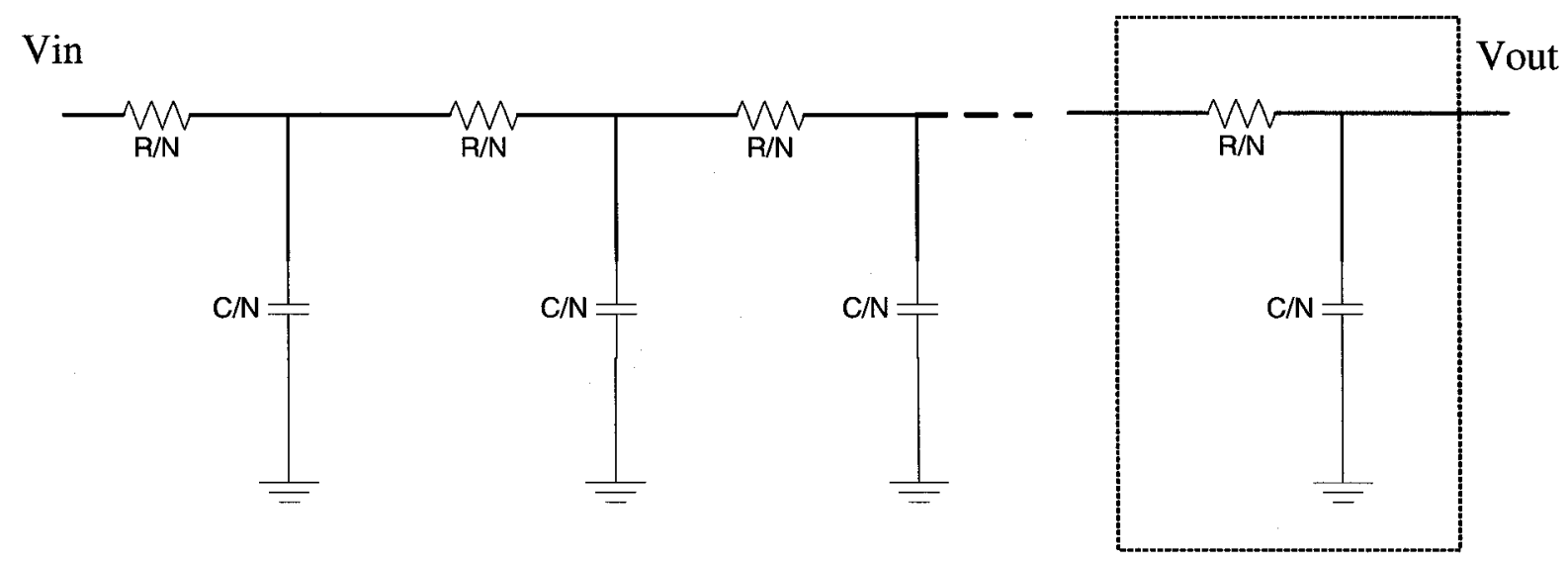

Figure 4.6 Distributed RC Ladder Network Model Consisting of N Equal Segments 
It is worth mentioning that the study in [26] [47] shows that the reduction of the average interconnection wire lengths will lead to an improvement in the throughput of analog decoders.

\section{Chip Area}

The primary difficulty during parallel LDPC decoder implementations is the complexity of the graph connectivity described by the $H$ matrix. Parallel decoder architectures directly map the nodes of a factor graph into processing elements, and the edges of the graph into an interconnecting network. The complexity is linear to their code length which originates from an inherently parallel nature.

Unlike in the cases of power consumption and delay, it is hard to find an analytic relation between chip area and interconnection wire length, but there turns out to be a useful empirical correlation between the two. The implementation results for some LDPC decoders with a parallel structure are listed in Table 4.1. According to the table, for different implementations, utilization rates are around $50 \%$ to $60 \%$. The utilization rate is low due to the long and complex interconnection wires that occupy a large amount of the chip area. With such a low utilization rate, an effective optimization in interconnection wires will significantly reduce the total wire length, which leaves room for further optimization to reduce the chip area. 


\begin{tabular}{|l|l|l|r|ll|}
\hline & & & & Core Area / Gates (digital) or \\
& Digital/Analog & Architecture & Utilization & Code & Total Transistors (analog) \\
\hline $1[24]$ & Digital & Parallel & $50 \%$ & $1024-\mathrm{b}$, rate 1/2, irregular code 7.5x7.0mm/1750k \\
$2[8]$ & Digital & Parallel & $50 \%$ & $1024-\mathrm{b}$, rate 1/2, irregular code 7.5x7.5mm/1750k \\
$3[22]$ & Digital & Parallel & $60 \%$ & $282-\mathrm{b}$, rate 1/2, irregular & $18 \mathrm{~mm}($ core) $/ 245 \mathrm{k}$ \\
$4[23]$ & Analog & Parallel & $60 \%$ & $(32,8,10)$ regular code & $690 \times 910 \mathrm{um} / 18.8 \mathrm{k}$ \\
\hline
\end{tabular}

\begin{tabular}{|l|l|l|l|}
\hline & Decoding Rate/Clock/Resolution & Technology & Remark \\
\hline $1[24]$ & $1 \mathrm{~Gb} / \mathrm{s} / 64 \mathrm{MHz} / 4 \mathrm{bits}$ & $220 \mathrm{~mW}, 5$ layers 0.16um 1.5V CMOS & Fabricated \\
$2[8]$ & $1 \mathrm{~Gb} / \mathrm{s} / 64 \mathrm{MHz} / 4 \mathrm{bits}$ & $690 \mathrm{~mW}, 5$ layers 0.16um 1.5V CMOS & Fabricated \\
$3[22]$ & $\mathrm{NA} / 100 \mathrm{MHz} / 4$ bits & 4 layers, 0.35um CMOS & IP core \\
$4[23]$ & $80 \mathrm{Mb} / \mathrm{s} / \mathrm{NA} / \mathrm{NA}$ & $5 \mathrm{~mW}, 6$ metal layers 0.18um 1.8V CMOS & Fabricated \\
\hline
\end{tabular}

Table 4-1 Utilization Rate for LDPC Parallel Decoders

In general, total interconnection wire length is a good overall indicator of placement "quality" and would make a useful optimization metric. (Here, "quality" is the measurement of optimization result that increases as delay times, power consumption and physical congestion problems lessen.) A good wire length minimization engine is therefore needed. It is believed that a timing- or congestionoriented approach, which evaluates the optimization effect based on critical path delay or severity of congestion (The critical path is the path that creates longest delay), can hardly be successful without a good wire length minimization engine. A placement with reduced total wire length tends to have a shorter critical time delay. Similarly, a good placement with optimized wire length has a higher probability of relatively smaller or less seriously congested regions. That means the routability is improved. Therefore, total wire length, which is equivalent to average wire length, will be used as a metric to evaluate the optimization result. 


\subsection{Input Files for FE (First Encounter) Generation}

The design process involves different types of files, which are generated and transferred among different software tools (see Figure 4.3). The tools involved include Matlab, Cadence Virtuoso, Cadence Abstract Generator, and Cadence First Encounter.

FE is a digital design tool developed by Cadence Corp for back end stage. Usually the physical implementation is called back end design. In a digital design, the situation is different from that of an analog design. Digital design involves many small standard cells, which usually come from a third party, e.g. a small design house. FE can handle placement and routing of these cells. During the placement process, cells which have physical interconnections tend to be placed relatively close to each other, although this placement result could be far from optimal. Also in a simpler situation where less number of cells, fewer kinds of processing units are involved, the placement result is better. That is the reason why iterative optimization gets a better optimization result by "fixing" one kind of cell while "placing" the other. Using our iterative optimization method, interconnection wire reduction mainly happens during the first two iterations.

\subsubsection{Automatic Structural Verilog Code Generation}

Hardware Description Language is a language used to describe a digital system; for example, a computer or a component of a computer. Verilog code and VHDL are two of the most popular hardware description languages (HDL). In this study, Verilog code was chosen for compatibility reasons, meaning it can be fully supported by FE. Verilog provides a rich set of built-in primitives at different abstraction levels, such as logical gates, switches, and user-defined primitives. Primitives are interconnected to form modules, which are in turn interconnected to form a design. The description of the connectivity of primitives and modules is called a structural description. Verilog also supports behav- 
ioral descriptions that model the functionality, as opposed to the gate-level connectivity, of a design. We need a structural Verilog as an input file for First Encounter.

A gate-level structural Verilog code looks like a netlist, which is essentially an enumeration of all of the circuit components (e.g. AND, OR, XOR gates). This structural Verilog code can be mapped directly from the parity check matrix, which describes the interconnections between different nodes. In order to smooth out the design flow, Verilog code should be generated automatically based on the $H$ matrix. It could be written manually. But in the case of LDPC codes with long block lengths, it will be very difficult or even impossible to handle it manually. An automatic Verilog code generation program takes the future cell library expansion into consideration, meaning that it can generate Verilog code for any LDPC code as long as certain rules are followed. In order to automate the generation of Verilog code, the names of the macro cells as well as the I/O pins need to follow certain rules. Details regarding the naming rules used in the Matlab program can be found in Appendix $C$.

We chose Matlab as the platform to automatically generate the Verilog code. Other program languages are applicable, such as C, Basic, or Java. The Matlab code can be found in Appendix A. A short sample code with an $H$ matrix (Figure 4.7) is shown below. Following the $H$ matrix is a sample Verilog code generated by our Matlab program.

$$
H=\left(\begin{array}{lll}
1 & 0 & 1 \\
1 & 1 & 1 \\
0 & 1 & 1
\end{array}\right)
$$

Figure 4.7 Sample $H$ Matrix 
The generated Verilog code with an $H$ matrix (Figure 4.7) is shown below.

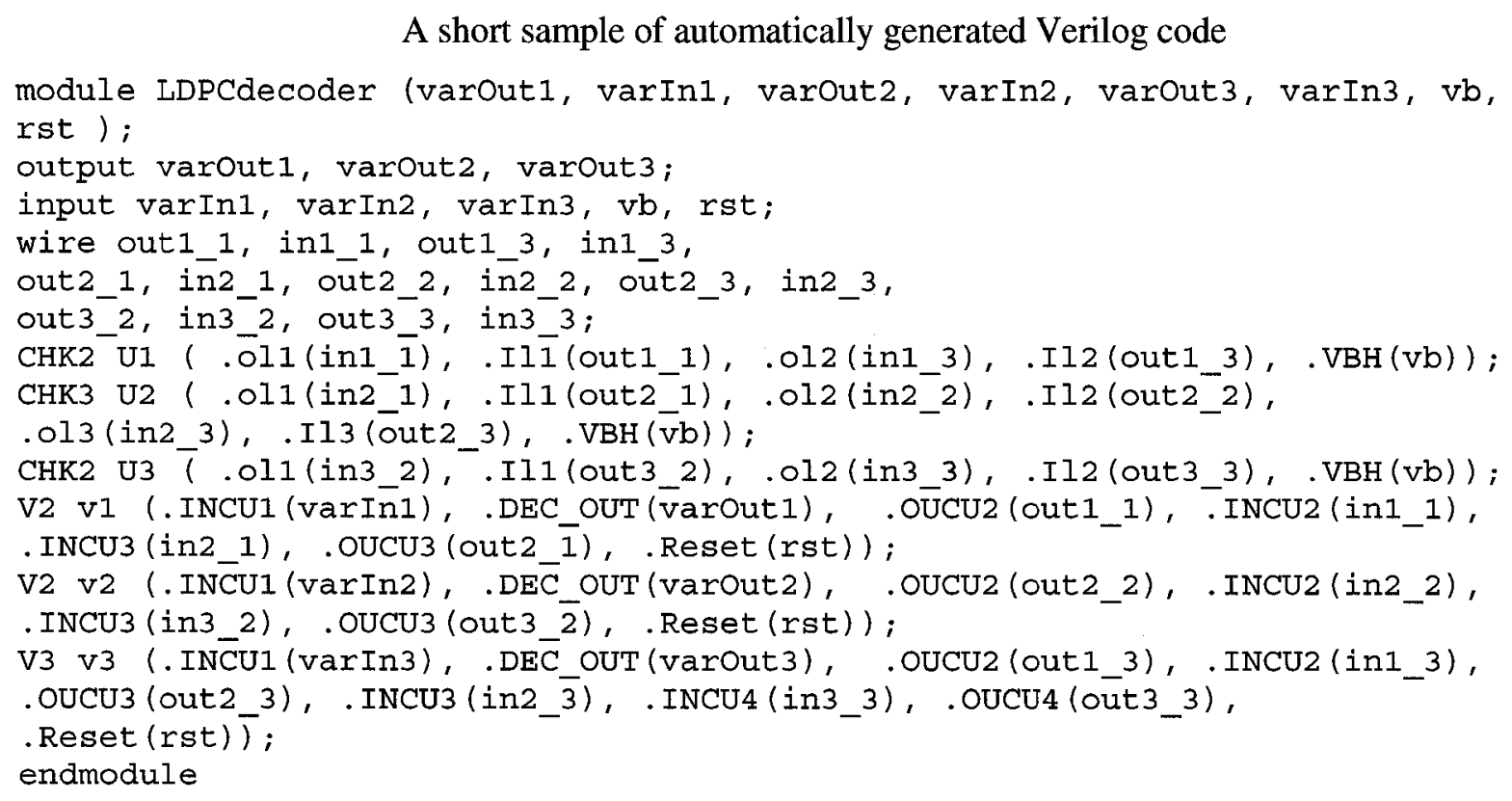

\subsubsection{Abstract View Generation \& LEF File}

After the Verilog code has been successfully generated, the cell Layouts view must then be changed to cell Abstracts view, which is a necessary step before the generation of a Library Exchange File (LEF). (An LEF is shown in Appendix E.). The Abstract view is essentially a cell layout without the silicon (active) layers. In other words, the Abstract version of a cell contains the cell geometry (x \& y), internal route obstructs (internal metal, polygons), and I/O pin locations. First Encounter will use Abstracts for placement \& route.

In order to create Abstracts, the layout of macro cells (check node and variable node) will be read into a tool called "Abstract Generator", which will convert macro cells into Abstracts view. First Encounter, which is a stand-alone tool, cannot read or generate Abstracts directly. An intermediate file that describes the various aspects of the design is needed, in an appropriate format. This kind of file is called a library exchange format file (LEF), which describes the cell's physical information. The 
physical information includes the size of the cell, the size and geometric position of the different physical layers (polygons, metal, via etc.), as well as the $\mathrm{I} / \mathrm{O}$ pin's geometric information.

When the Abstract generation is finished, the LEF file can be exported from the Abstract Generator. The exported LEF file might contain technology information. Usually, an independent technology file is preferred, to avoid possible conflicts due to dual-specifications. The technology information should be deleted from the LEF file. A small part of a sample technology file can be found in Appendix D at the end of this thesis.

\subsubsection{Technology File}

Like the LEF file, the technology file has a "lef" extension but contains different information. A standard technology file contains the definitions of the various layers, the design rules, the display properties, and the extraction parameters required to view, design, simulate and fabricate the design. The information can be included in the LEF file, but it is usually put in the independent technology file. The technology used in our case is six layers, CMOP18 provided by TSMC.

\subsection{Placement \& Routing: Iterative Optimization Method}

The placement is realized by the "cell placement" command in FE. After FE finishes the placement, we find some cells have been placed in "incorrect" locations (this means the interconnection wire is long), even when a much better position is available. This is probably due to the complexity of the interconnection network, which results in the built-in placement algorithm of FE giving out an unsatisfactory result. We also observed the phenomenon that if fewer cells are involved, FE can handle the 
placement much better. It is understandable that optimization efficiency will be improved as long as the complexity is reduced.

Note that parallel LDPC decoders have the following characteristic features:

1) There are only two categories of nodes (check node and variable node).

2) There are no interconnections inside these two categories of nodes. All the information exchange (physical connections) happens between the check nodes and variable nodes.

3) The nodes are assumed to be pre-optimized and may be treated as "black boxes" in our optimization.

During the placement process, it is normal practice to have a "floor plan" first. In our case, we have a floor plan that assigns two areas for two kinds of macro cells (the check node and the variable node). What we don't know is how to find an optimized location for a specific macro cell under different interconnection networks (or $H$ matrix). Our iterative optimization method is proposed for solving this problem.

Iterative optimization method description:

1) After loading all the files, initiate a placement by FE. All the macro cells are placed (all cells mixed in the core area). After this step, the initial specific position of a cell can be assigned by the designer according to the floor plan.

2) Start with the check nodes. Set all the check nodes properties to "Fixed", leaving the properties of the variable nodes "Unplaced". This means that the check nodes will not change location if FE replaces the macro cells. 
3) Execute the FE placement command. Note that the variable nodes move to new positions while the check nodes stay where they are. Some variable nodes may be placed at positions, which do not meet requirement of floor plan. In that case, manually move these variable nodes to nearest available positions.

4) Run "Tryroute" to check wire length.

5) Set the properties of the variable nodes to "Fixed", while resetting the properties of the check nodes to "Unplaced".

6) Execute the FE placement command again. This time note that the check nodes are moved to new positions while the variable nodes stay where they are. Same like variable nodes, some check nodes may be placed in positions, which do not meet the requirement of floor plan. Manually move these check nodes to nearest available positions.

7) Run Tryroute again to check wire length.

8) Iterate this optimization process until no significant improvement in wire length is observed.

9) The final stage will be real "routing" using the "wroute" command. 


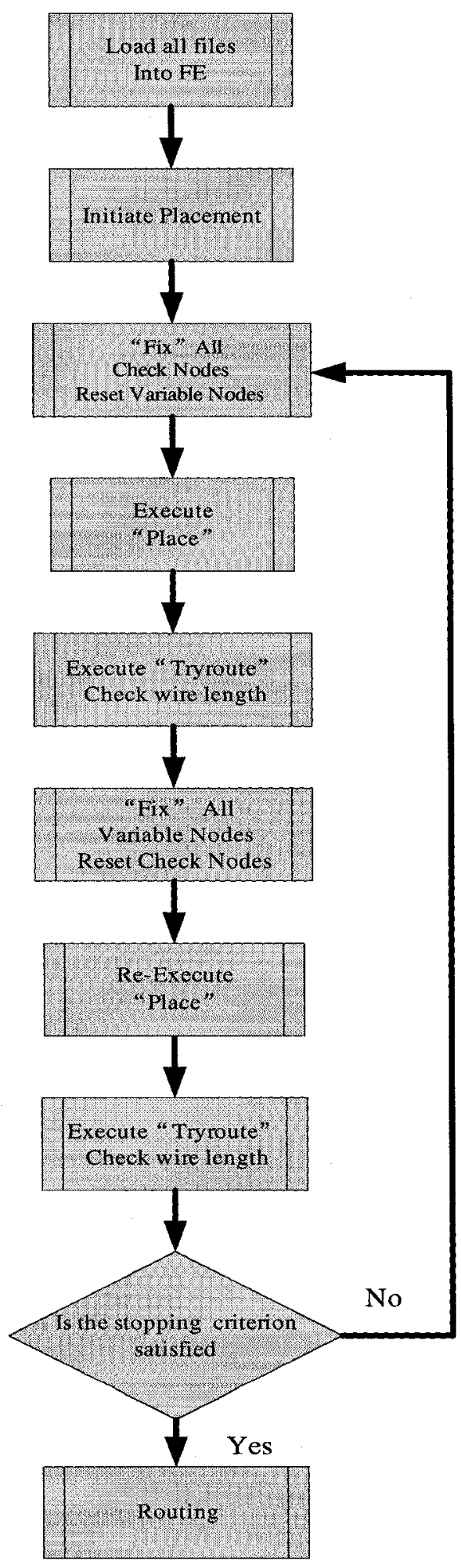

Figure 4.8 Iterative Optimization Method Using First Encounter 


\subsection{Case Study \& Analysis}

To demonstrate the optimization results of our method, we present two designs with pre-built macro cells (degree-4 check nodes and degree-3 variable nodes). For comparison purposes, one design has a floor plan similar to our fabricated decoder, which was designed following a traditional analog design flow. The iterative optimization method was checked further by supplying it with a floor plan that differs from the one above. And finally we will use a complex case (an $H$ matrix of $210 \times 280$ ) to show the effectiveness of our optimization method for practical codes. The results will be compared with each other. The $H$ Matrix of the $(32,8,10)$ LDPC code is shown below.

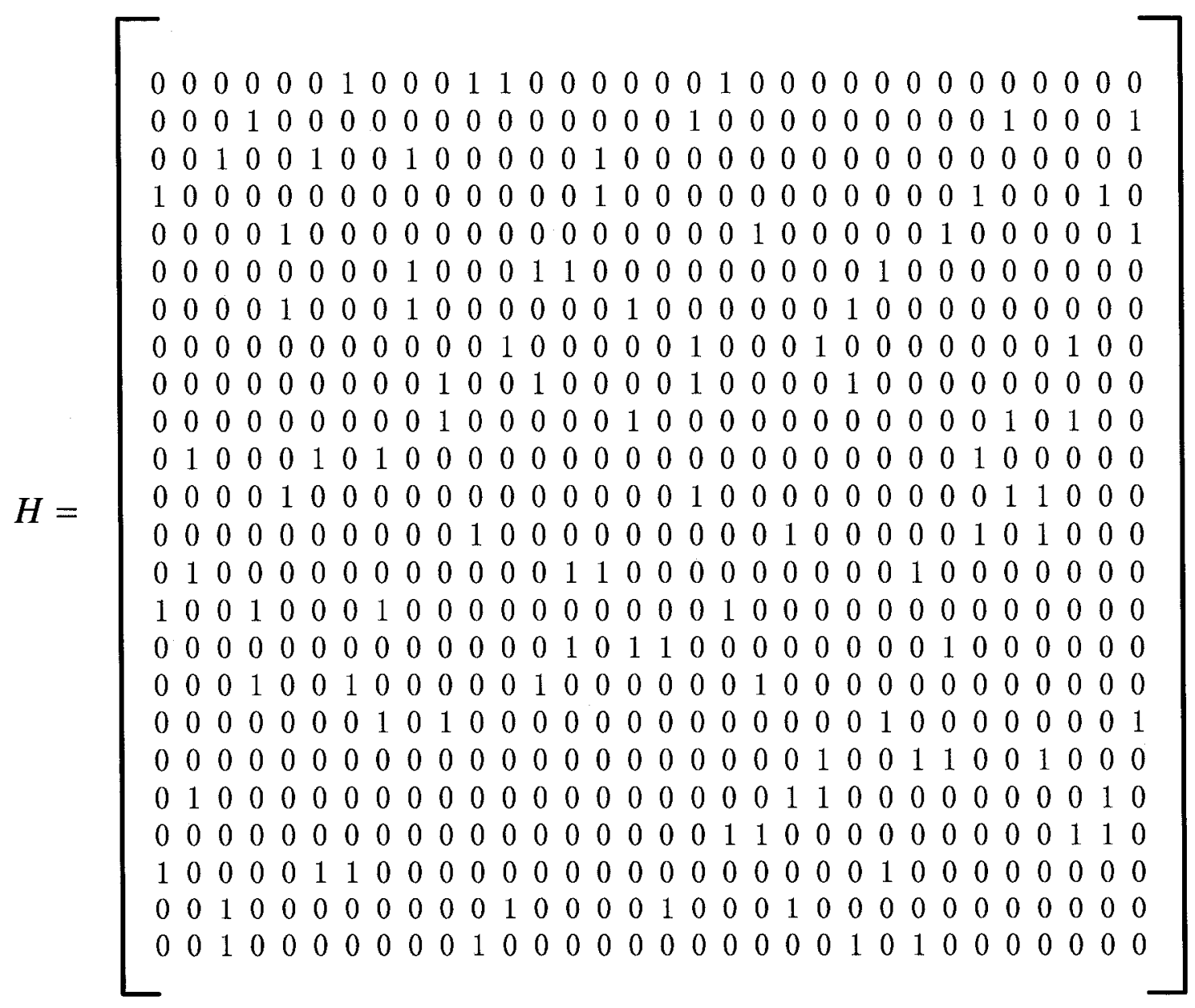

Figure 4.9 $H$ Matrix of a $(32,8,10)$ Regular LDPC Code 


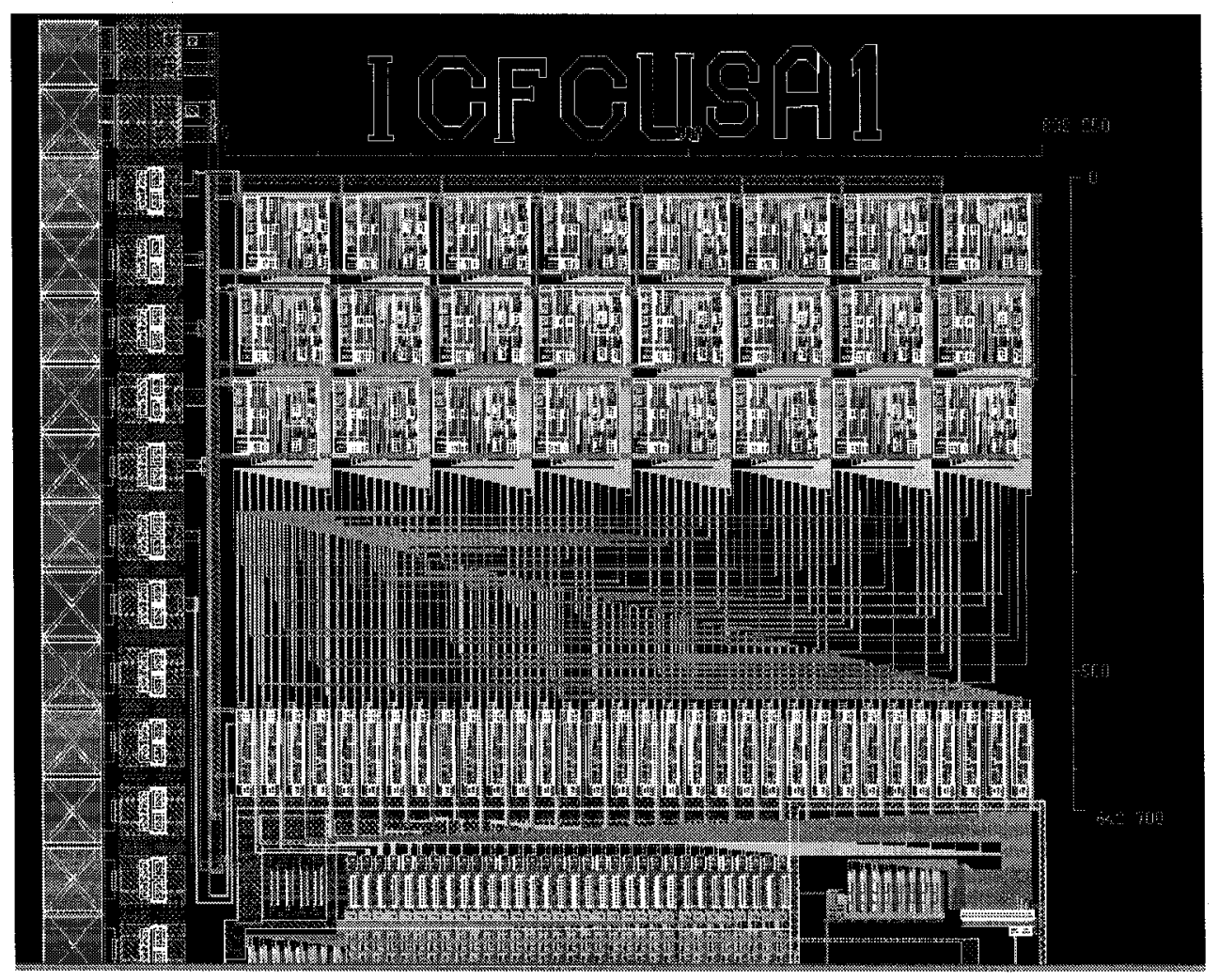

Figure 4.10 Layout of Analog Decoder for $(32,8,10)$ LDPC Code Following Traditional Design Flow [26]

For the benchmark following a traditional design flow, we get (referring to [26]) Core Area (area for variable nodes, check nodes and routing):

$$
A=880 \times 640=56320=5.63 \times 10^{4} \mu m^{2}
$$

Roughly half the area is used for interconnection wires.

Total wire length (internal interconnection wire only) referring to [26]:

$$
\begin{aligned}
L & =450 \times 36+550 \times 55+680 \times 38+800 \times 23+920 \times 18+1030 \times 12+1150 \times 10 \\
& =131110=1.31 \times 10^{5} \mu \mathrm{m}
\end{aligned}
$$


The internal interconnection wire refers to the interconnection wire between variable nodes and check nodes. These wires are most important in our optimization since the throughput of the decoder is heavily affected by the total internal wire length, which equates to the average wire length (average constant time delay) [26] [47].

\section{Case 1}

In this case, a placement profile exactly the same as the one in our benchmark is used as our starting point for the iterative optimization process for convenience in comparing the results. Using the same profile means the macro cells are placed in a location relatively similar to our benchmark. The total core area is also identical in both cases, although the height/width is a little bit different. The reason for this is that while the Abstracts are being generated, a guard region is needed, which is defined by a shape called "Prbound". This guard region is used to prevent design rule violations from occurring during the placement. The presence of the guard region makes the Abstract a little bigger than the layout.

After the initial placement, the iterative optimization method is applied in order to find an optimized placement. After an optimized placement is found, power routing and interconnection wire routing follow. The optimized macro cells placement profile is shown in Figure 4.11. 


\section{I/O Pins}

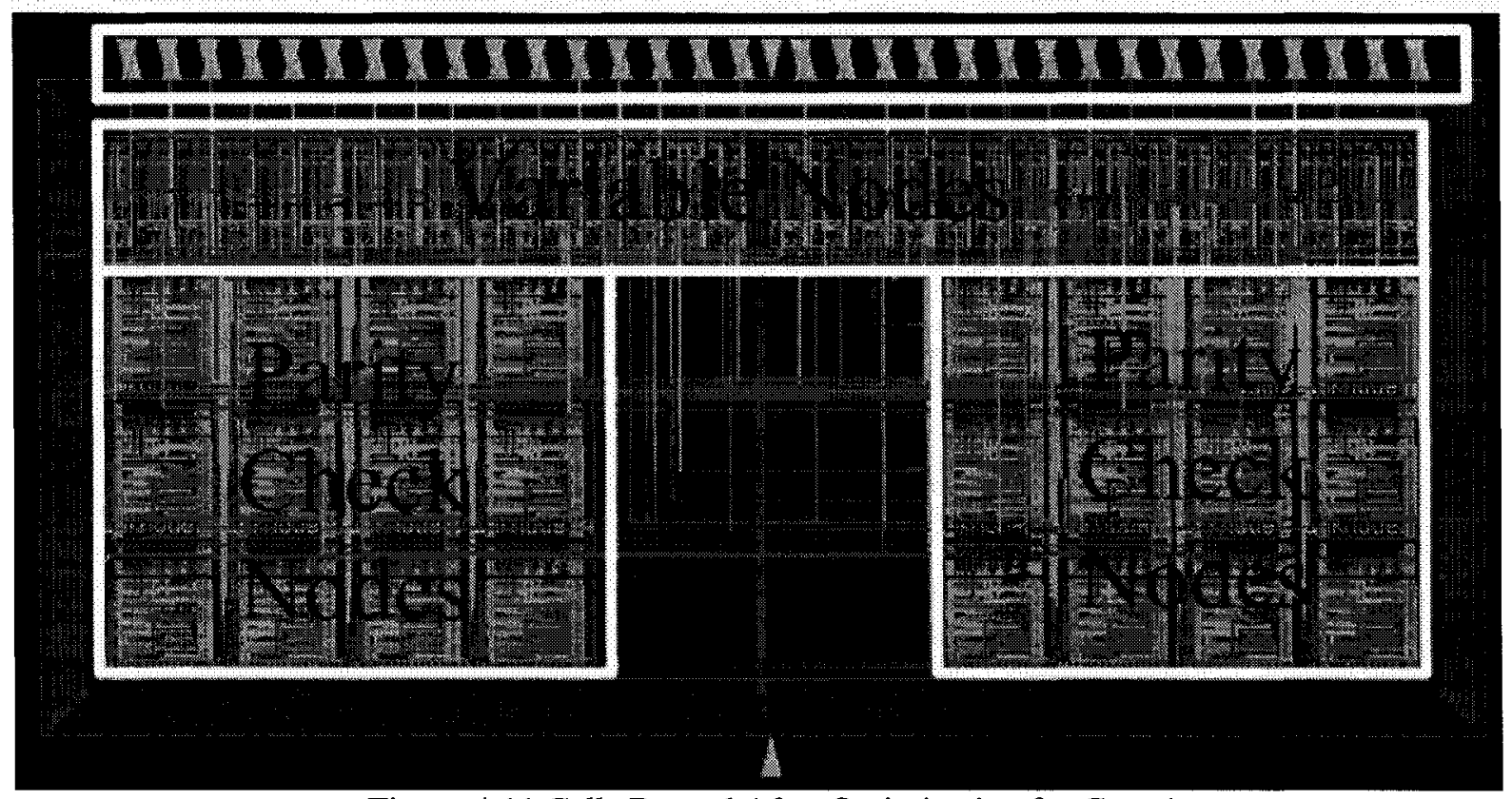

Figure 4.11 Cells Routed After Optimization for Case 1

Compared to our benchmark, there is an empty area in the middle of our core. This is due to the guard area of the variable node. The width of the guard area is about $4.3 \mathrm{um}$, while there are 32 variable nodes in a row, the total length used in the guard area is $L 1=32 \times 2 \times 4.3=275.2 \mathrm{um}$. At the same time, there are 8 check nodes in a row. The total length used in the guard area is $L 2=8 \times 2 \times 4.3=68.8 \mathrm{um}$. This situation is due to the discrepancy between L1 and L2. 


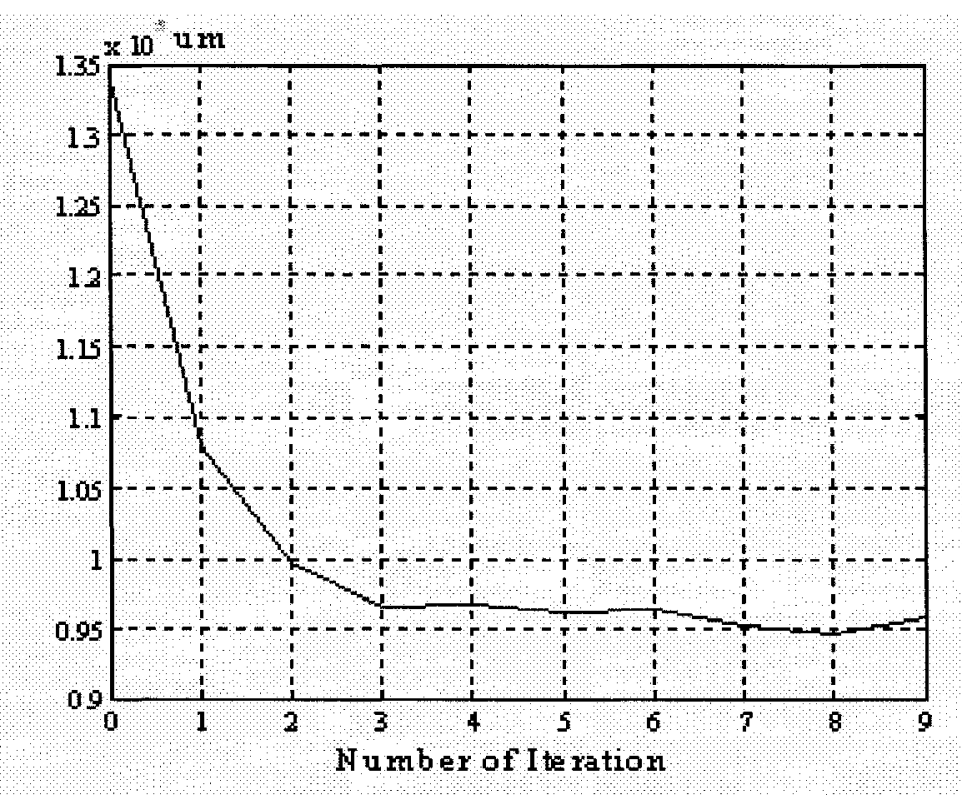

Figure 4-12 Wire Length vs. Iteration

During the iterative optimization process, the internal interconnection wire length decreases from $1.33 \times 10^{5} \mu \mathrm{m}$ to $0.95 \times 10^{5} \mu \mathrm{m}$. The iterative optimization gives us a saving of more than $30 \%$ in internal interconnection wire length. Referring to formulas (4.5) and (4.6), that means a $30 \%$ saving in the power consumption of the interconnections, and the RC delay is reduced by nearly $50 \%$. Research [26] [47] show that average time constant is inversely proportional to the throughput. Based on this conclusion, the throughput will accordingly increase by $100 \%$.

FE is an area router rather than a channel router, which means that the interconnection wire can go through any areas on the chip if necessary. In contrast, in the case of channel routing, a special area is assigned for routing exclusively. In our case, since the LDPC code is relatively small, no special rout- 
ing area is needed. This fact (no special routing area needed) implies that the utilization rate can be much higher compared to the cases in Table 4.1.

Using the same floor plan, we also check the optimization result of $\mathrm{FE}$ in a situation if both variable nodes and check nodes are placed in one shot rather than using our iterative optimization method. The check nodes and variable nodes whose positions violate the floor plan will be manually moved to proper positions. The optimization result (table 4.2) of FE is similar to our randomly initial placement. It should be noted that if one tries to place and rout both the variable nodes and check nodes in one shot using FE, one would not be able to enforce non-overlapping floor plans for variable nodes and check nodes, as two different groups of nodes. This would result in a random mixture of check nodes and variable nodes with many floor plan violations.

\section{Case 2}

We propose Case 2, using a different floor plan. In this floor plan, all the check nodes are allocated to the central area, while the variable nodes are placed in the ring area around the IP (intellectual property) core. The variable node is also turned by $90^{\circ}$. Since we don't know the optimized placement profile at the beginning, we place both check nodes and variable nodes in an "orderly" way. For a definition of "orderly", please refer to Figure 4.14. The check nodes are placed sequentially in 3 rows with number 1 to 8,9 to 16,17 to 24 respectively, while variable nodes are placed sequentially in the ring area starting from 1 to 32 . We then start our iterative optimization method. 


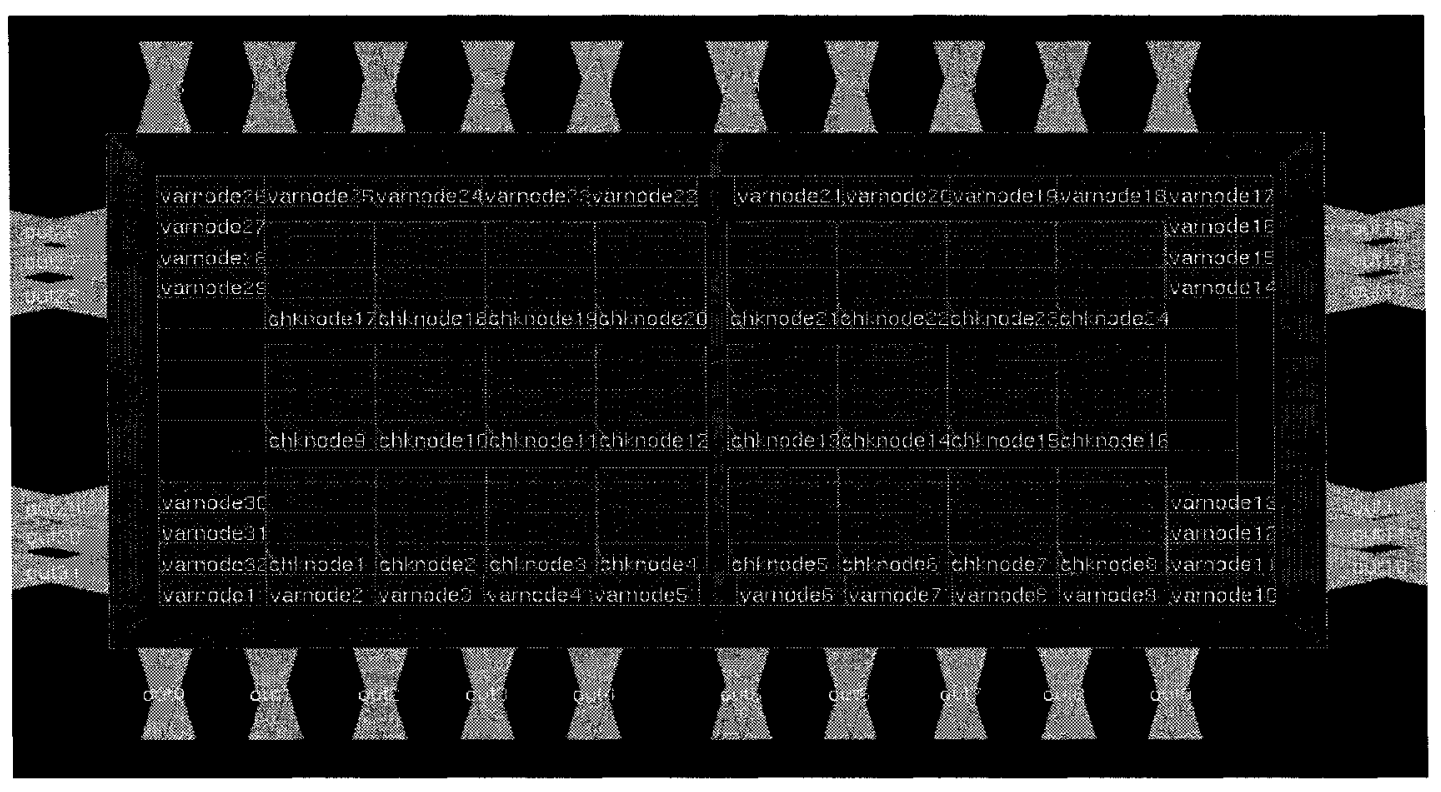

Figure 4.13 Initial Floor Plan \& Placement for Case 2

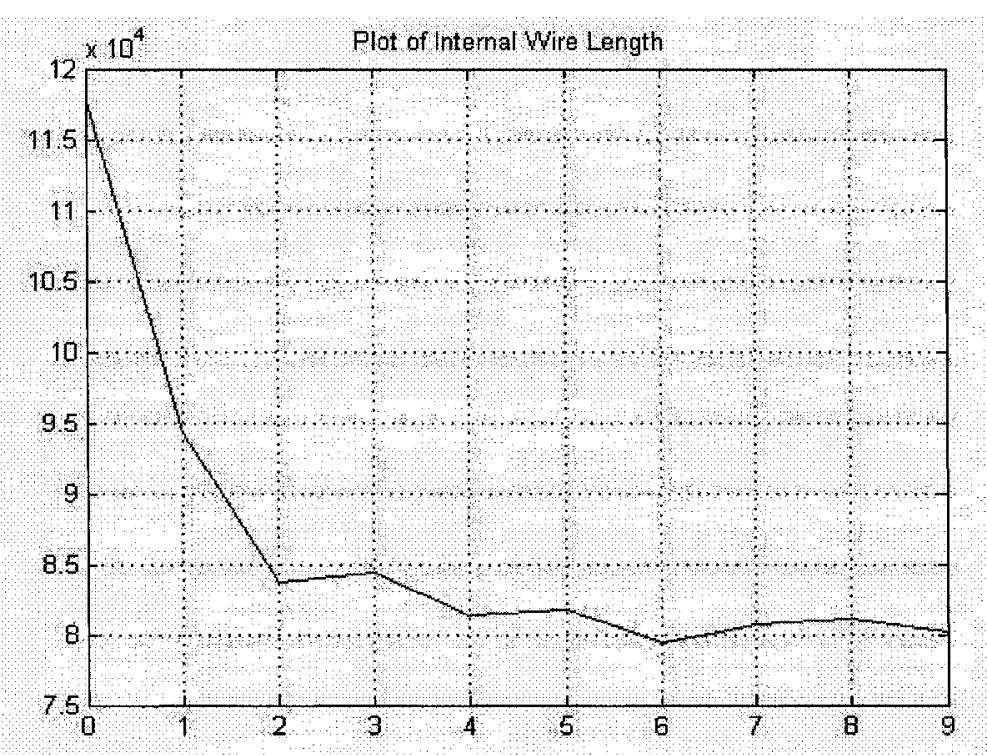

Figure 4.14 Internal Wire Length vs. Iteration for Case 2 
During the optimization process, the internal wire length decreases from $11.7 \times 10^{4} \mu m$ to around $8 \times 10^{4} \mu \mathrm{m}$, a decrease of $31 \%$. Compare with case 1 , the core area of case 2 decreases slightly (around 1\%), but the optimized internal wire length is $16 \%$ better than optimized result of case1 (Case1: $0.95 \times 10^{5} \mu m$ vs.Case2: $8 \times 10^{4} \mu m$ ) The optimization result shows the placement figure which influence internal wire length.

\section{Case 3}

In this case, the iterative optimization method is applied to an LDPC decoder for a regular code with a $210 \times 280 H$ matrix. The LDPC code is too complex for a designer to handle manually, but as can be seen, our design flow can handle it very well and the optimization result is $23 \%$ better than initial internal wire length, which is impressive.

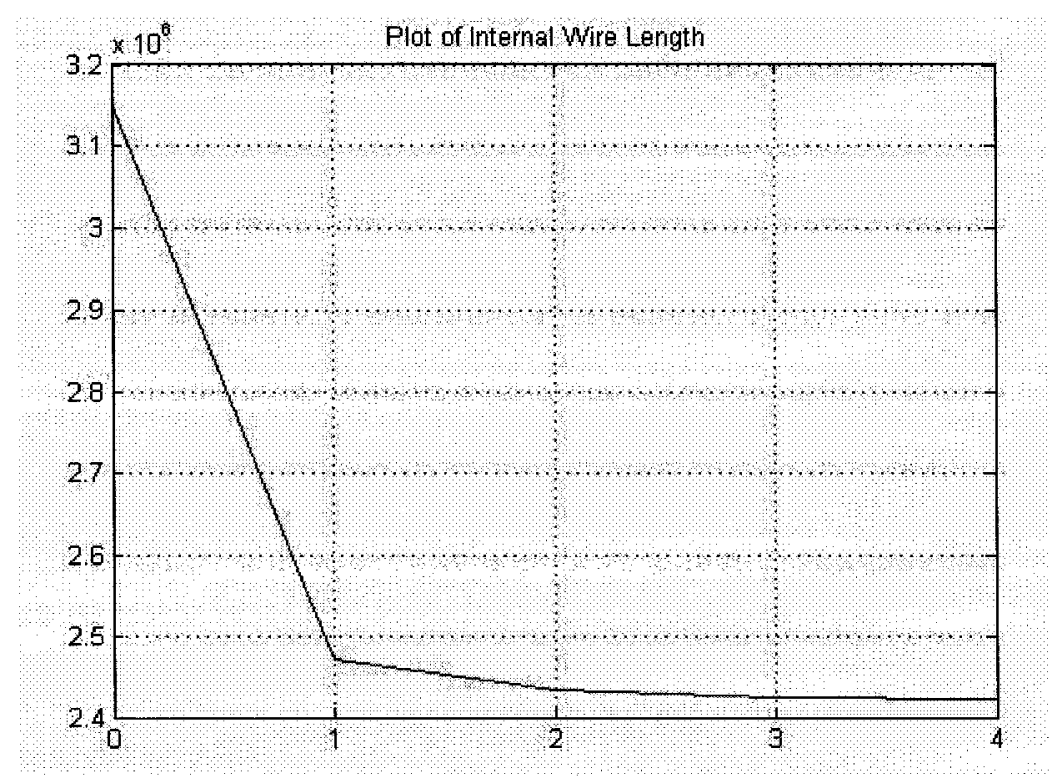

Figure 4.15 Internal Wire Length vs Iteration for Case 3 


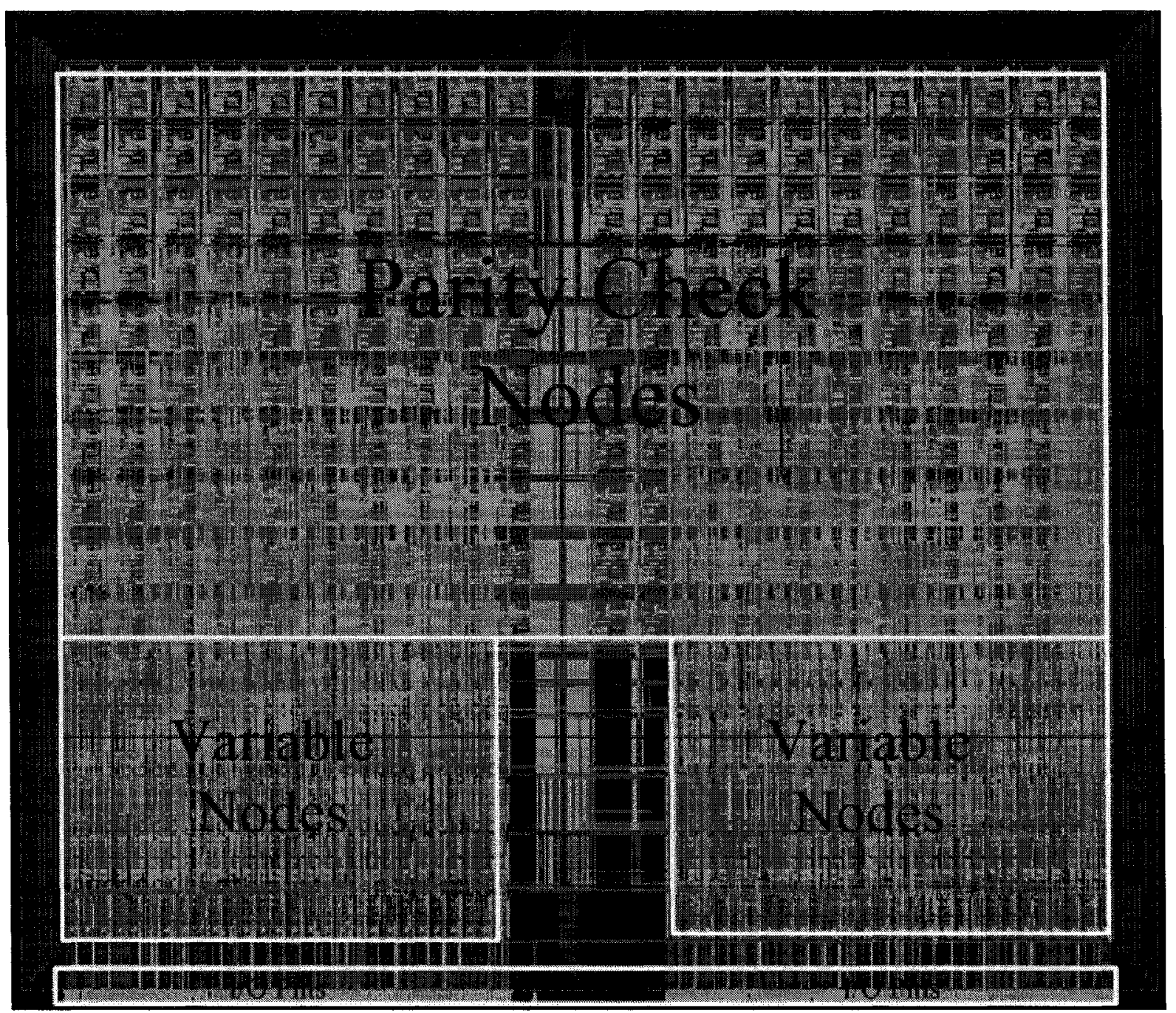

Figure 4.16 Layout of IP Core with $H$ Matrix $(280 \times 210)$

\begin{tabular}{|l|r|r|r|r|}
\hline Benchmark & Core Area(um^2) & $\begin{array}{l}\text { Initial Internal } \\
\text { Wire Length } \\
1.30 \mathrm{E}+05\end{array}$ & $\begin{array}{l}\text { Optimized Internal } \\
\text { Wire Length }\end{array}$ & Improvement \\
\hline Case 1(Iterative Optimization) & $5.63 \mathrm{E}+05$ & $1.33 \mathrm{E}+05$ & $9.50 \mathrm{E}+04$ & $29 \%$ \\
\hline Case 1(Place by FE, in one shot) & & N/A & $1.32 \mathrm{E}+05$ & N/A \\
\hline Case 2 & $5.47 \mathrm{E}+05$ & $1.17 \mathrm{E}+05$ & $8.00 \mathrm{E}+04$ & $31 \%$ \\
\hline Case 3 & $4.47 \mathrm{E}+06$ & $3.15 \mathrm{E}+06$ & $2.42 \mathrm{E}+06$ & $23 \%$ \\
\hline
\end{tabular}

Table 4.2 Optimization Effect Comparison for Different Cases 
For the 3 cases above, in general the iterative optimization method achieves $20 \%$ to $30 \%$ optimization result compare with our random initial placement. For the best case (case 2), the optimization result is $31 \%$ better than initial number. Since both case 1 and case 2 have same parity check matrix with similar core area, the different optimized finial results mean placement configuration can help to achieve a better optimization result. This direction is worth further study in future.

\subsection{Complexity vs. Length of LDPC Codes}

For LDPC codes, we know the decoding complexity increases linearly as code length increases. The following will study the impact of code length on iterative optimization method. Using the same prebuilt cells, we will study 3 more cases, code length 64,128 and 500 respectively plus our previous 2 cases (code length 32 and code length 280). The placement time and routing time will be plotted and compared. Floor plans for these three new cases similar to case 1 and case 3 are showed below. Cells in all cases are initially placed in an orderly fashion, like the previous cases.

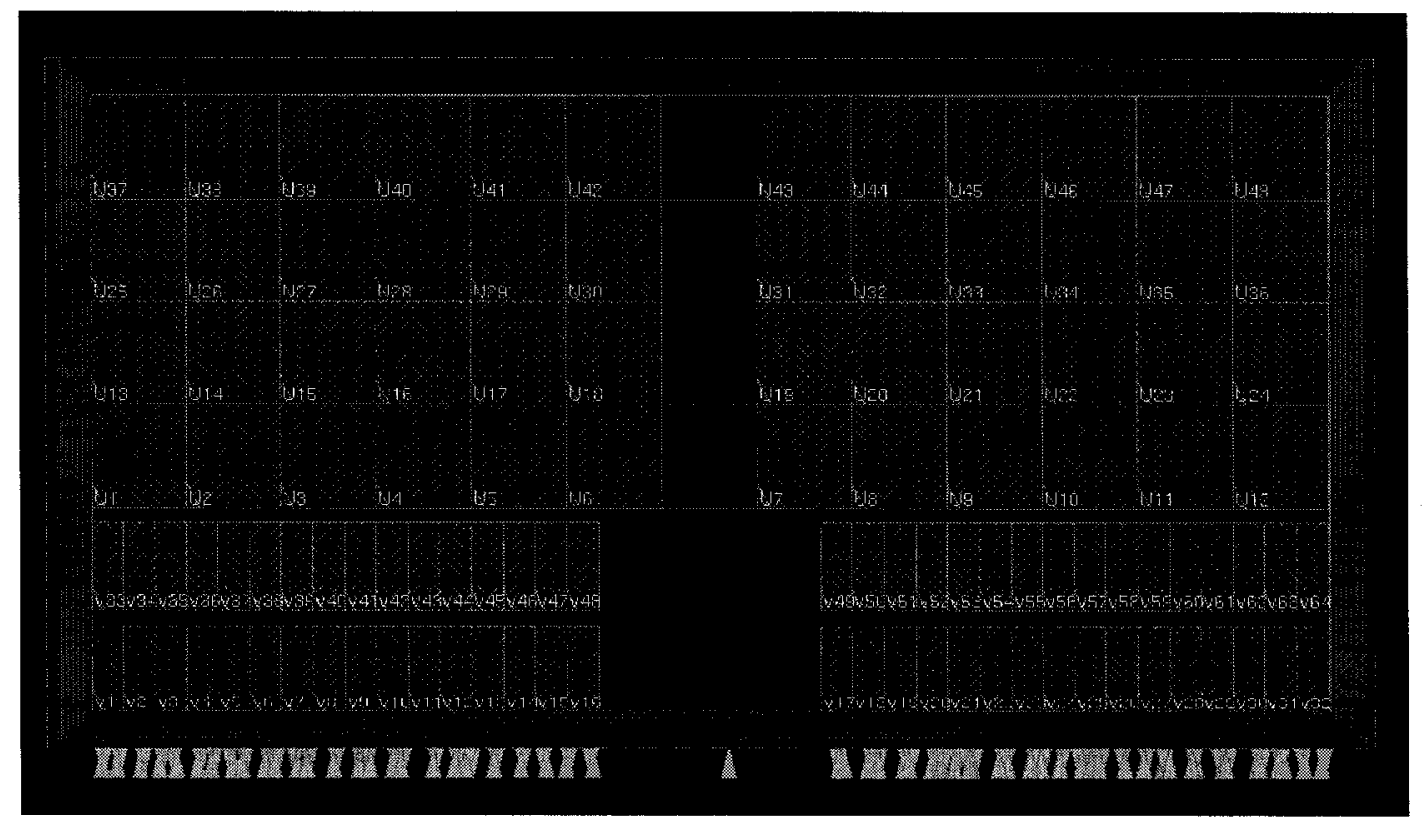

Figure 4.17 Floor Plan for LDPC code with Code Length 64 


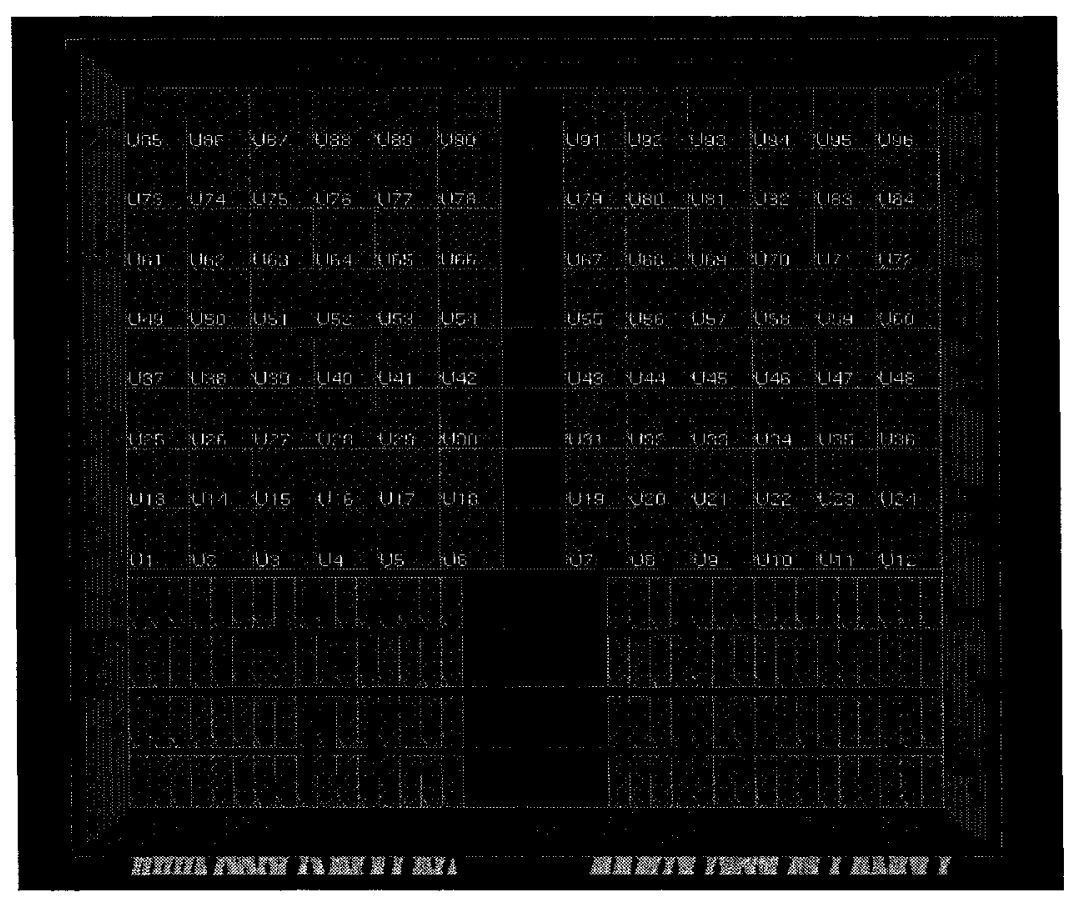

Figure 4.18 Floor Plan for LDPC Code with Code Length 128

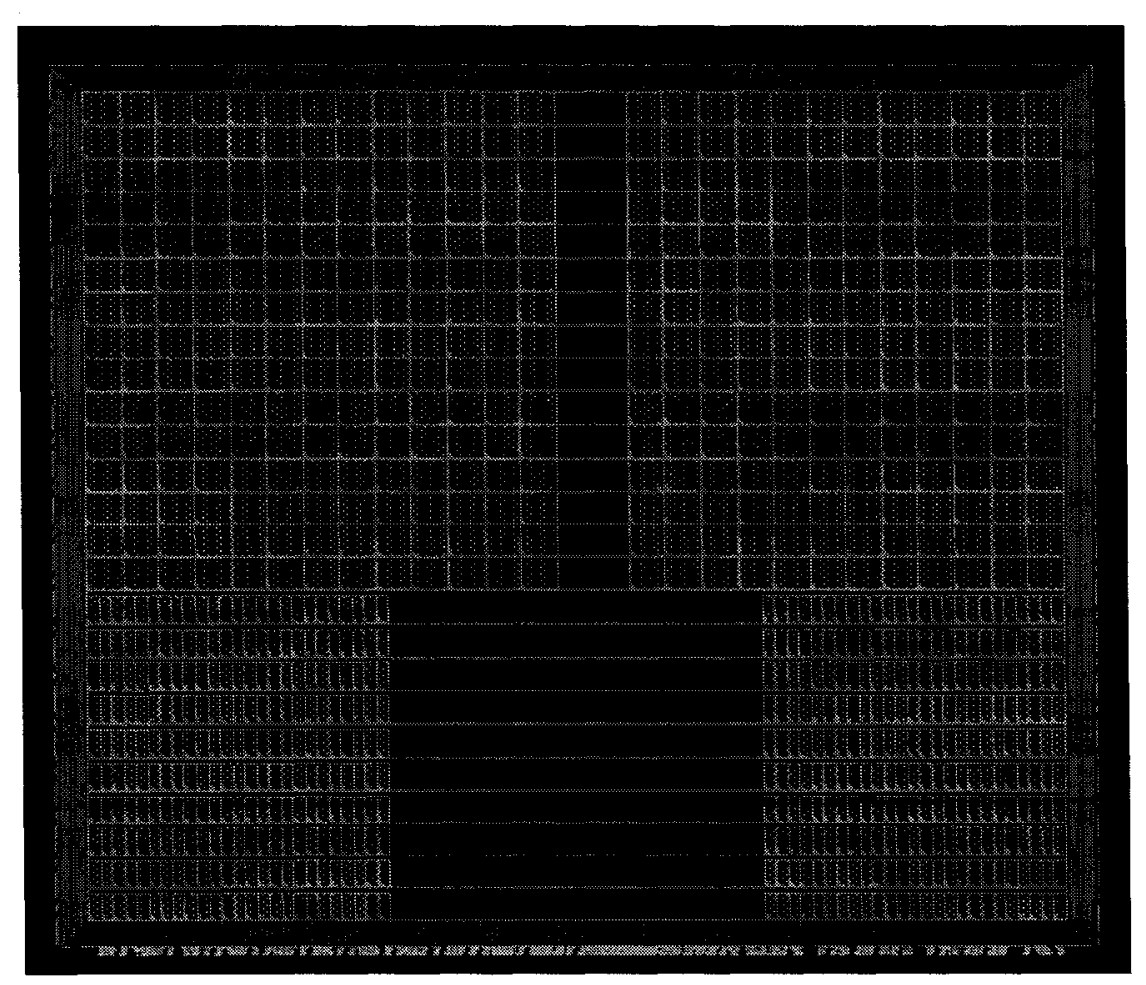

Figure 4.19 Floor Plan for LDPC Code with Code Length 500

68 
The placement time and routing time needed by FE for LDPC codes with different lengths are shown in Figure 4.20. The placement time is defined as the total time needed by the computer to execute the "place" command in 4 rounds because the optimization effect diminishes after that. The "place" command includes the placement of variable nodes and check nodes. After executing the "place" command, some cells (check nodes or variable nodes) may be placed at positions, which do not meet the requirement of the floor plan. In that case, we manually move those cells to the nearest available positions. The time for manual movement is excluded from placement time. Figure 4.20 clearly shows "routing" time and "place" time track each other although routing takes much longer time than placement. As the code length increases, the time for both placement and routing increases dramatically.

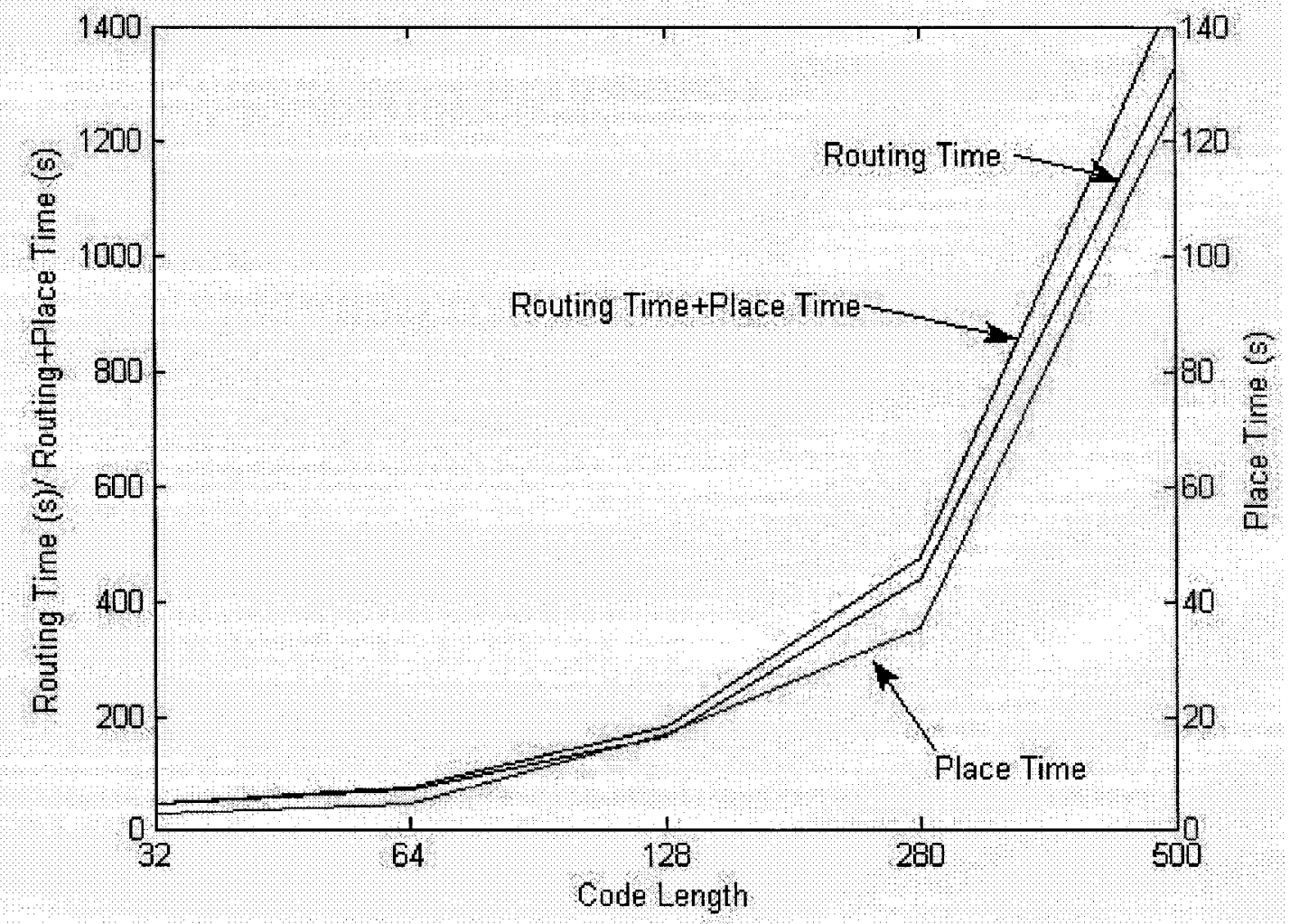

Figure 4.20 Complexity of Iterative Optimization vs. Code Length 


\subsection{Other Related Issues}

Once placement and routing is finished, various physical checks are carried out.

\subsubsection{Physical Check}

1) Layout vs. Schematic (LVS)

The LVS check is done by "Verify Connectivity". This function extracts the connectivity information from the routed layout and compares it with the final logical netlist, which is Verilog code in our case. An LVS match confirms that errors were not introduced during the physical layout of the design. This verification can detect and report various conditions, such as open nets, antennas, loops, and partial routing for all nets or specified nets in the design.

\section{2) Design Rule Check (DRC)}

The design rule check is done by "Verify Geometry". This function verifies the physical layout of design, such as ensuring that widths, spacing and internal geometry of objects in the design meet the requirements of the foundry. The foundry refers to the fabrication facility, or fab, in which the integrated circuits are manufactured. TSMC is an example of a foundry.

\section{3) Antenna Check}

When a router uses a metal layer to connect to the input pins, long metal routing causes an electrical charge accumulation during the fabrication etching process. If there is no discharge path for the metal that is connected directly to the transistor gate, the built-up voltage may become large enough to damage the gate oxide. This is called the process antenna effect (PAE). To determine the extent of PAE, the router calculates the Antenna Ratio, which is defined as the area of the metal layer relative to the area of the gates connected to it. 


\section{Antenna Ratio $=\frac{\text { Area of Metal Layer }}{\text { Area of Gates }}$}

Each foundry sets a maximum allowable antenna ratio for the chip it fabricates.

Two methods of avoiding PAE:

1) Changing the routing so the total area of any of the layers connected to a gate is not so large that it builds enough static charge to damage the gate.

2) Inserting diodes that protect the gate by providing an alternate path to discharge the static charge.

The antenna check is done by "Verify Process Antenna".

\section{4) Power connection}

The power line is connected manually. The reason we manually route power and ground lines is not because FE cannot handle this automatically. It is because the pre-built cells do not meet the requirement of standard cells. In FE, the power line is regarded as a special signal. The way FE handles it is to wire the power line straight rather than zigzag like wire for general signal. As long as these cells are built in the same way as standard cells, FE can handle power line automatically. 


\subsubsection{Power Planning}

The essence of power planning is connecting the VDD and GND rails of the macro cells through lowresistance lines to the external supply. When designing the power grid for the circuit, the following two rules should be kept in mind:

1) In order to avoid electro-migration, keep the average current density below the limits of the technology. In the case of CMOSP18, the limits are $1 \mathrm{~mA} / \mathrm{um}$ of wire width for M1 through M5 and $1.6 \mathrm{~mA} / \mathrm{um}$ for $\mathrm{M} 6$ at temperature $110^{\circ} \mathrm{C}$.

2) Prevent IR drop from adversely affecting circuit performance. IR drop is caused when a rush of current is drawn through a resistive line, causing a temporary supply voltage (or IR) drop. Based on the design's speed, layout, and toggling activity, power rails must be distributed across the design to maintain this limit. An effort should be made to keep IR drop negligible-generally lower than $5 \%$ of the supply voltage. For example, at $1.8 \mathrm{~V}, 5 \%$ is $90 \mathrm{mV}(1.8 \mathrm{~V} \times 5 \%=90 \mathrm{mV})$.

In order to design the power supply network, a rough estimate of the circuit's power and current consumption is needed. The average total power consumption for the fabricated chip is $5 \mathrm{mw}$. The average current $I_{a v g}=\frac{5 m w}{1.8 v}=2.8 m A$. Considering there are three pairs of independent power supply $\left(V_{d d}, V_{s s}, V_{d d-d i g}, V_{s s-d i g}, V_{d d-s u b}, V_{s s-s u b}\right)$, the width of the power rings is chosen to be 5um, which will leave us enough of a safety margin for the current density. 
Taking the IR drop into consideration, the worst case IR drop for Case 1 , which has only 1 pair of connection points to the ideal supply (usually, there are several pairs of connections to the ideal power supply), will be

$$
R=\frac{l}{w_{1}} R_{s q m 1}+\frac{l}{w_{2}} R_{s q u m 2}+R_{V I A}
$$

Referring to the process documentation, $R_{s q m x}=0.08, w_{1}=w_{2}=5 u m, R_{V I A}=6 \Omega$

$$
l=2 \times\left(\text { width }_{\text {core }}+\text { heigh } h_{\text {core }}\right)=3196 u m
$$

This results in

$$
R=108 \Omega
$$

We will choose 4 pairs $(\mathrm{N}=4)$ of connections to the ideal power supplies. In that case, the maximum IR drop will be

$$
V_{I R_{-} d r o p}=\frac{R \times I_{\text {avg }}}{N^{2}}=\frac{108 \times 2.8}{4^{2}}=18.9 \mathrm{mV}
$$

The maximum permitted IR drop is $1.8 \mathrm{~V} \times 5 \%=90 \mathrm{mV}$, so four pairs of connections to the ideal power supply distributed around the power ring will satisfy the IR drop requirement. This design gives us a sufficient safety margin in the power supply.

\subsubsection{Floor Planning Considerations}

As technologies become smaller, delay due to interconnection resistance and capacitance becomes more significant than gate delay. We have borrowed the concept of gate delay, which is used in digital circuits. Gate delay refers to the time required for a logic gate's output to reach $50 \%$ of its final output level when a change occurs on the input(s). Reducing gate delays in digital circuits allows them to process data at a faster rate and improve overall performance. Therefore, if two cells are 
physically beside each other, they will experience much less delay than if they are separated by the length of the chip. As technology advances and the size of the transistor reduces dramatically, gate delay decreases and the impact of RC delay becomes more significant. A good floor plan will help reduce this delay. As is obvious in our Case 2, the floor plan contributes to the optimized result, since both cases have a slightly different IP core size (less than $2 \%$ difference), but the final optimized result gives us a roughly $15 \%$ difference.

It should be noted that in our case, the sizes of the check node and the variable node differ both in width and height. The difference in size limits our flexibility regarding floor planning. For this reason, we try to put the same kind of cell in a limited region in an aligned arrangement. When the heights are the same, chip area is saved, compared to when cells of different heights are mixed together.

With the floor plan, it is also important to pay attention to the ease with which the design can be understood, modified and reused by other designers, which can easily be overlooked. A design style that is easy to understand is always important to and appreciated by a team. Our sample design is a decoder IP core, which can be reused by others. A well-organized floor plan helps with an organized I/O arrangement and problem tracking, as well as helping others to follow what has been done. 


\section{Chapter 5 Conclusion and Future Work}

In this chapter, some conclusions are provided. Future work is also put forward and discussed.

\subsection{Summary}

Analog LDPC decoders are becoming more attractive in recent years compared with their digital counterparts. The main motivation for implementing an iterative decoder in an analog domain instead of the traditional digital circuit is to improve the speed/power ratio and minimize the chip area while maintaining the intrinsically better error-correcting performance [26] of an analog decoder. However, the traditional analog design flow is not suitable for analog LDPC decoder design. The problem arises from the difficulty of manually routing and placement during the design. Even short, regular LDPC codes (less than 100 bits) are hard to implement following a traditional design flow. For long irregular LDPC codes, which usually outperform their regular counterparts, it is almost impossible. Due to the complexity of the interconnecting networks, the analog LDPC decoder design is far from perfect and there is plenty of room to improve. Based on the research [26] [47] that propagation delay is the dominant delay factor in analog iterative decoders, our discussion on optimization metrics leads us to the conclusion that total interconnection wire length can be a good optimization effect indicator. With this conclusion, in this thesis a design flow is suggested based on the previous work (pre-built cell library) in our group and an iterative optimization method is proposed and used in this design flow. In the three cases described in the thesis, the proposed iterative optimization method shows promising results with dramatically decreased interconnection wire length. This optimized result means higher throughput, less chip area, improved energy efficiency. 


\subsection{Future Work}

Although a great deal of progress has been made in recent years, research on analog LDPC decoders is a relatively new area. Questions ranging from system level design and simulation, circuit design and implementation to fabrication technology need to be answered before volume production can start. A few topics of interest are listed here.

\section{Optimization of Analog Decoders with Multi-degrees Variable/Check Nodes}

Although the optimization results are promising, our work is based on a relatively simple case, regular LDPC codes with two kinds of macro cells (a degree-4 check node processing element and a degree-3 variable node processing element). In order to use this method in practical design, which is usually much more complicated, further exploration in this area is needed.

In practical applications, it is not uncommon to have check nodes and variable nodes with different degrees, whose sizes and shapes vary considerably. For multi-degree variable/check nodes, size can vary dramatically, from $1 \mathrm{x}$ the size of a smallest macro cell to as large as $10 \mathrm{x}$. In analog LDPC decoder core design, although we may not have a hundred different macro cells, there can definitely be a large swing in size as degree increases. Placing macros of varying sizes without good optimization can result in fragmentation of placement and routing space, making the design unroutable.

A future study can be conducted in situations with multi-degree macro cells and irregular code with long block length to apply this iterative optimization method. 


\section{The Effect of Different Placement Pattern}

VLSI floor planning is an important stage in chip design. A typical approach to floor plan design is to determine the topology (i.e., the relative positions of the modules) of the floor plan primarily using the interconnection information among the modules. It involves the placement of a set of rectangular circuit modules (macro cells) on a chip so as to minimize the total area and the total interconnection wire length. Obviously a different placement configuration will affect the final optimization result. The work in this thesis is based on a given placement pattern while searching for the optimized position for a specific cell. The effect of applying our iterative optimization method to different placement patterns has not been studied thoroughly. Further study may be needed in this direction. 


\section{References}

[1] C.E. Shannon, "Communication in the presence of noise", Proceedings of the IEEE, Vol. 72, Issue, 9, Sept. 1984, pp 1192-1201.

[2] C. Berrou, A. Glavieux, and P. Thitimajshima, "Near Shannon limit error-correcting codes and decoding", Proc. Int. Conf. Comm. '93, May 1993, pp 1064-1070.

[3] R.G. Gallager, "Low Density Parity Check Code", IRE Transactions on Information Theory, Vol. IT-8, Jan. 1962, pp. 21-28.

[4] T. Richarson, M. A. Shokrollahi and R. Urbanke, "Design of Capacity-Approaching Irregular Low-Density Parity-Check Codes", IEEE Transactions on Information Theory, Vol. 47, No. 2, Feb. 2001, pp. 619-637.

[5] M. G. Luby, M. Mitzenmacher, M. A. Shokrollahi, and D. A. Spielman, "Improved Low-Density Parity-Check Codes Using Irregular Graphs", IEEE Transactions on Information Theory, 47(2), Feb 2001, pp.585-598.

[6]K. Gunnam and G Choi, "A Low Power Architecture for Min-Sum Decoding of LDPC Codes." Available at http://dropzone.tamu.edu/techpubs/2006/TAMU-ECE-2006-02.pdf.

[7] K. Shimizu, T. Ishikawa, N. Togama, T. Ikenaga and S. Goto, "Partially-parallel LDPC Decoder Achieving High-Efficiency Message-passing Schedule", IEICE Transactions on Fundamentals of Electronics, Communications and Computer Sciences 2006 E89-A(4), pp. 969-978.

[8] A. J. Blanksby and C. J. Howland. "A 690-mw 1024-b, Rate 1/2 Low-density Parity-check Code Decoder", IEEE Journal of Solid-state Circuits, 37(3), 2002.

[9] Y. Kou, Slin, and M. P. C. Fossorier, "Low-Density Parity-Check Codes Based on finite Geometries: A Rediscovery and New Results", IEEE Trans. Info. Theory, vol. 47, no.7, Nov. 2001, pp. 2711-36.

[10] E. Yeo et al, "High Throughput Low-Density Parity-Check Architectures", Proc. IEEE GLOBECOM. San Antonio. TX, Nov. 25-29, 2001, pp. 3019-24.

[11] C. A. Mead, "Silicon Models of Neural Computation", in Proc. IEEE $1^{\text {st }}$ Int. Conf on Neural Networks, Vol. 1, June 1987, pp. 91-106.

[12] C. A. Mead, Analog VLSI and Neural Systems, Reading: Addison Wesley, 1989.

[13] M. Mansour and N.Shanbhag. "Architecture Aware Low Density Parity-Check codes", IEEE Int. Symp. Circuits and Systems, May 2003.

[14] M. Mansour and N. Shanbhag. "A Novel Design Methodology for High Performance Programmable Decoder core for AA-LDPC Codes", IEEE Workshop on Signal Processing Systems, Aug. 2003.

[15] J. Thorpe. "Design of LDPC Graphs for Hardware Implementation", In IEEE Int. Symp. Inform. Theory, Lausanne, Switzerland, June 2002, p. 483. 
[16] J. Campeliot, D.S. Modha, and S. Rajagopalan. "Designing LDPC Codes Using Bit-Filling”, In IEEE Int. Conf. Communications, June 2001, pp. 55-59.

[17] J. Dai. Design Methodology for Analog VLSI Implementations of Error Control Decoders, PhD Thesis, University of Utah, 2001.

[18] F. Lustenberger, On the Design of Analog VLSI Iterative Decoders, PhD Thesis, Swiss Federal Institute of Technology, Zurich, 2000.

[19] J. Dai, Winstead, C. J. Myers, R.R. Harrison, and C. Schlegel. "Cell Library for Automatic Synthesis of Analog Error Control", In Circuits and Systems, 2002. ISCAS 2002. IEEE International Symposium, Vol 4, pp. 481-484.

[20] J. Rogers, and C. Plett, Radio Frequency Integrated Circuit Design, Artech House Publishers, 2003.

[21] S. M. Kang, and Y. Leblebici, CMOS Digital Integrated Circuits Analysis and Design, Third Edition, McGraw Hill, 2003.

[22] G. Murphy, E. M. Popovici, R. Bresnan, W. P. Marnane and P. Fitzpatrick, "Design and Implementation of a Parameterizable LDPC Decoder IP Core", IEEE $24^{\text {th }}$ International Conference on Microelectronics, Nis Serbia and Montenegro, Vol. 2, May 2004, pp. 747-750.

[23] S. Hemati, A.H. Banihashemi, and C. Plett, "An 80-Mb/s 0.18um CMOS Analog Min-Sum Iterative Decoder for a $(32,8,10)$ LDPC Code", Proc. IEEE Custom Integrated Circuit Conf., San Jose, CA, Sep 2005.

[24] C. Howland and A. Blanksby, "A 220mW 1Gb/s 1024-bit Rate-1/2 Low Density Parity Check Code Decoder", IEEE Custom Integrated Circuit Conference, San Diego, CA, May 2001.

[25] Manual from Cadence Corp, Encounter User Guide, Technology File and Display Resource File ASICII Syntax Ref Manual, Encounter Design Flow Guide, Encounter Text Command Ref.

[26] S. Hemati, Iterative Decoding in Analog VLSI, PhD Thesis, Ottawa-Carleton Institute for Electrical and Computer Engineering, Carleton University, 2005.

[27] T. Bhatt, K.Narayanan, and N. Kehtarnavaz, "Fixed Point DSP Implementation of Low Density Parity Check Codes," Proc. IEEE DSP2000, Hunt, TX, Oct. 15-18, 2000.

[28] T. Zhang and K. Parhi, "A 57Mb/s (3, 6)-Regular FPGA LDPC Decoder", Proc. IEEE SIPS 2002, San Diego, CA, Oct. 16-18, 2002, pp. 127-32.

[29] C. Winstead, N. Nguyen, V.C. Gaudet, and C. Schlegel, "Low-Voltage CMOS Circuit for Analog Decoders", April 2006, IEEE transactions on Circuits and System I: Regular Papers, Vol. 53, Issue4, pp. 829-841.

[30] D. J. C. MacKay and R. M. Neal, "Near Shannon Limit Performance of Low Density Parity Check Codes", appeared in Electronic Letters.

[31] H. Zhong and T. Zhang. "Design of VLSI implementation-oriented LDPC codes", in Proc. IEEE Vehicular Technology Conference, 2003, pp. 670-673. 
[32] T. Zhang and K. Parhi. "Joint (3,k)-regular LDPC code and decoder/encoder design", IEEE Transactions on Signal Processing, 52(4), 2004, pp. 1065-1079.

[33] V. Nagarajan, N. Jayakumar, S. Khatri, and O. Milenkovic, "High-throughput VLSI implementations of iterative decoders and related code construction problems," in Proc. IEEE GLOBECOM 2004, vol. 1, Dec. 2004, pp. 361-365.

[34] S. Hemati, A. H. Banihashemi and C. Plett, "A 0.18-um CMOS Analog Min-Sum Iterative Decoder for a $(32,8)$ Low-Density Parity-Check (LDPC) Code," IEEE Journal of Solid-States Circuits, vol. 41, No. 11, pp. 2531-2540, Nov. 2006.

[35] R. M. Tanner, "A Recursive Approach to Low Complexity Codes," IEEE Trans, Inform Theory. vol.IT-27. Sept .1981, pp. 533-547.

[36] N. Wiberg, Codes and Decoding on General Graphs. Ph.D. Dissertation. Linköping University. Sweden, 1996. Available at http://www.it.isy.liu.se/publicationer/LIU-TEK-THESIS-440.pdf.

[37] M. R. Yazdani, S. Hemati, and A. H. Banihashemi, "Improving Belief Propagation on Graphs with Cycles", IEEE Comm. Lett., vol. 8, no.1, Jan. 2004., pp. 57-59

[38] M. Fossorier, M. Mihaljevic, and H. Imai, "Reduced complexity iterative decoding of low density parity check codes based on belief propagation," IEEE Transactions on Communications, vol. 47, May 1999, pp. 673-680.

[39] J. Zhao, F. Zarkeshvari, and A. H. Banihashemi, "On Implementation of Min-Sum Algorithm and its Modifications for Decoding LDPC codes," IEEE Trans. Comm, vol. 53, no.4, April 2005, pp. 549-554.

[40] J. Chen and M. P. C. Fossorier, "Density Evolution for Two Improved BP-Based Decoding Algorithms of LDPC Codes," IEEE Comm. Lett., vol.6, no.5, May 2002, pp. 208-210.

[41] T. Brack, M. Alles, F. Kienle and N. When, "A Synthesizable IP Core for Wimax802.16E LDPC Code Decoding," Available at http://www.eit.uni-kl.de/wehn/files/publikationen/2006/PIMRC06.pdf

[42] IEEE 802.16 Broadband Wireless Access Working Group, "LDPC coding for OFDMA PHY”, Available at http://grouper.ieee.org/groups/802//16/tge/contrib/C80216e-04 373r1.pdf

[43] Dielissen. J, Hekstra. A, Berg.V, "Low Cost LDPC Decoder for DVB-S2", Design, Automation and Test in Europe, vol. 2, 6-10 March 2006, pp 1-6

[44] Bates. S, Iniewski. K, "10 GBPS over Copper Lines-State of the Art in VLSI”, System-on-Chip for Real Time Application, 2005 Proceedings, Fifth International Workshop, July 2005, pp. 491-494

[45] M. Fossorier, "Iterative Reliability Based Decoding of Low-density Parity Check Code," IEEE J. Select. Areas Communications, vol. 19, pp. 908-917, May 2001

[46] Robert H. Morelos-Zaragoza, Art of Error Control Coding, Second Edition John Wiley \& Sons, 2006.

[47] S. Hemati and A. H. Banihashemi, "Convergence speed and throughput of analog decoders," accepted for publication in IEEE Trans. Comm., March 2006 
[48] H. T Siegelmann, Neural Networks and Analog Computation: Beyond the Turing Limit, Birkhanser, Boston, 1999 


\section{Appendix A Matlab Code}

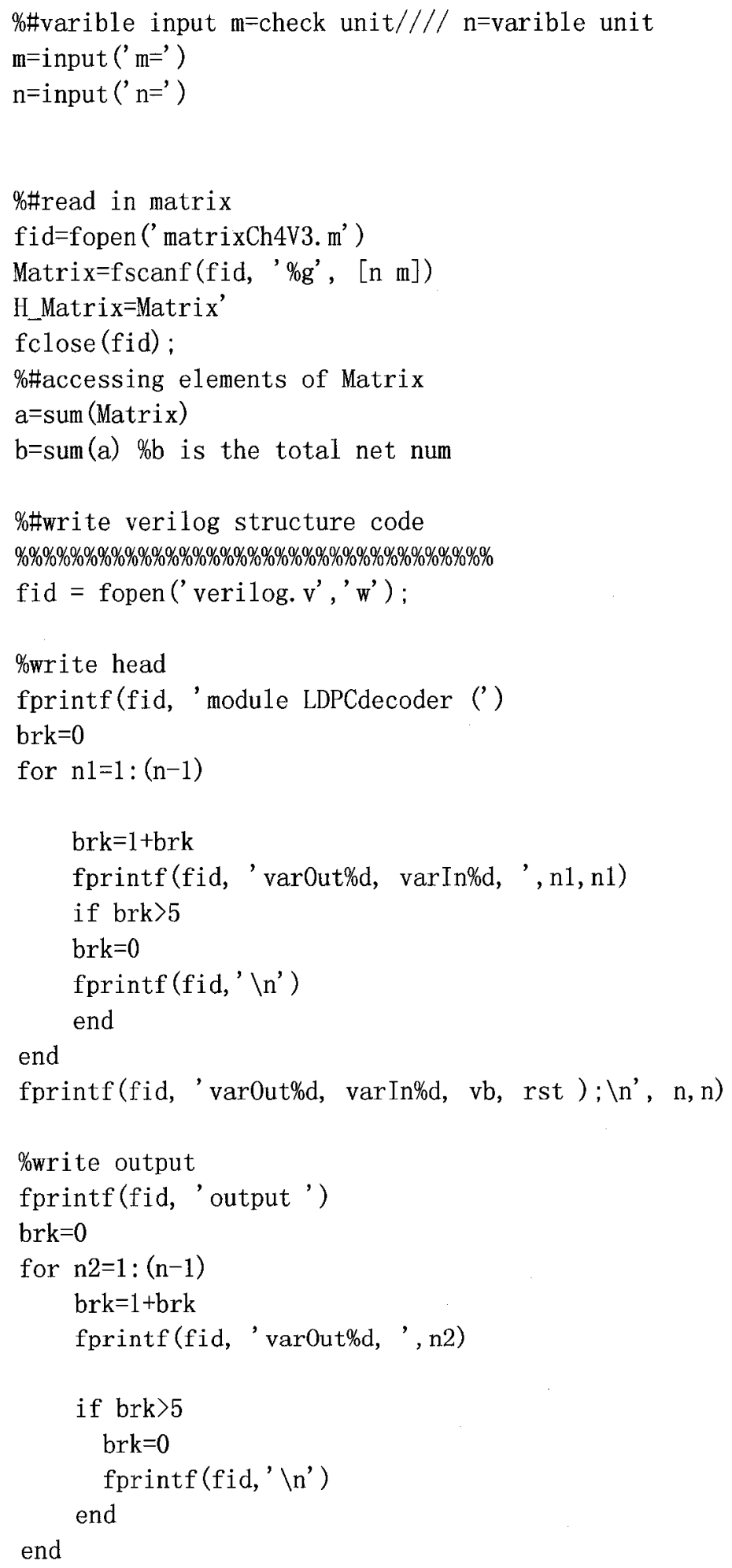


fprintf(fid, 'var0ut\%d; \n', n)

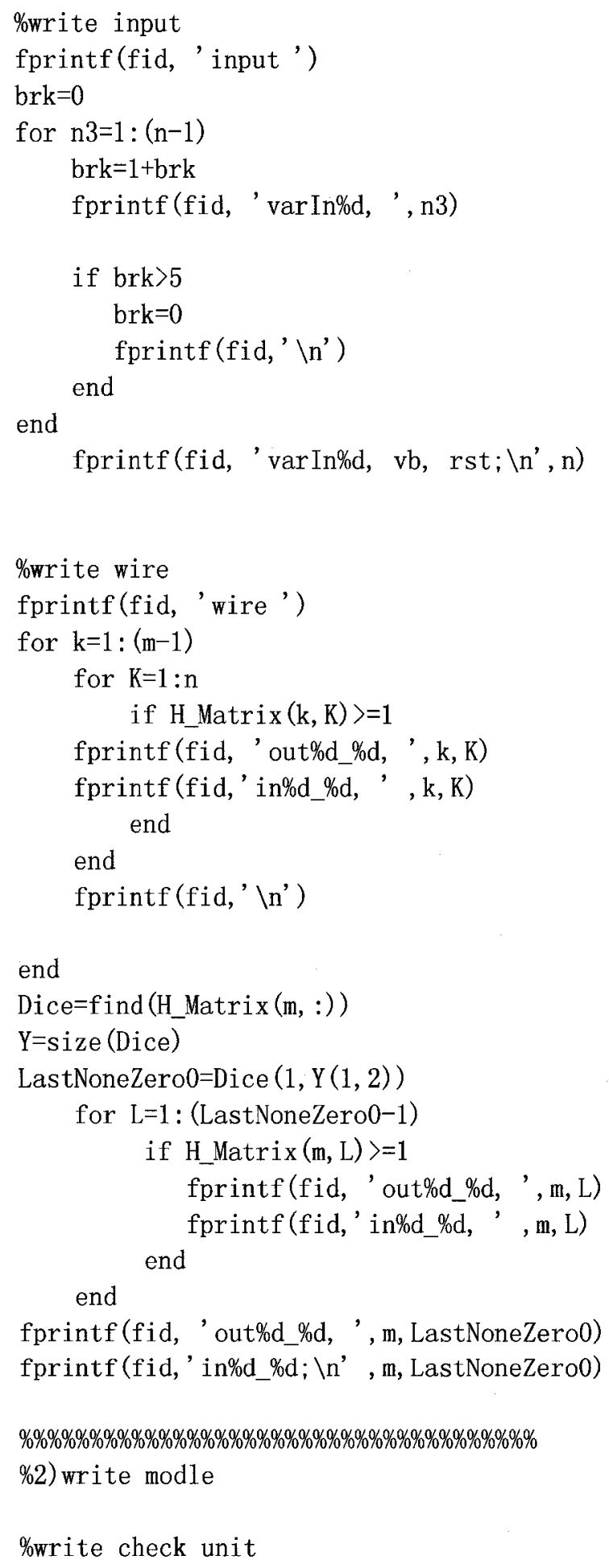




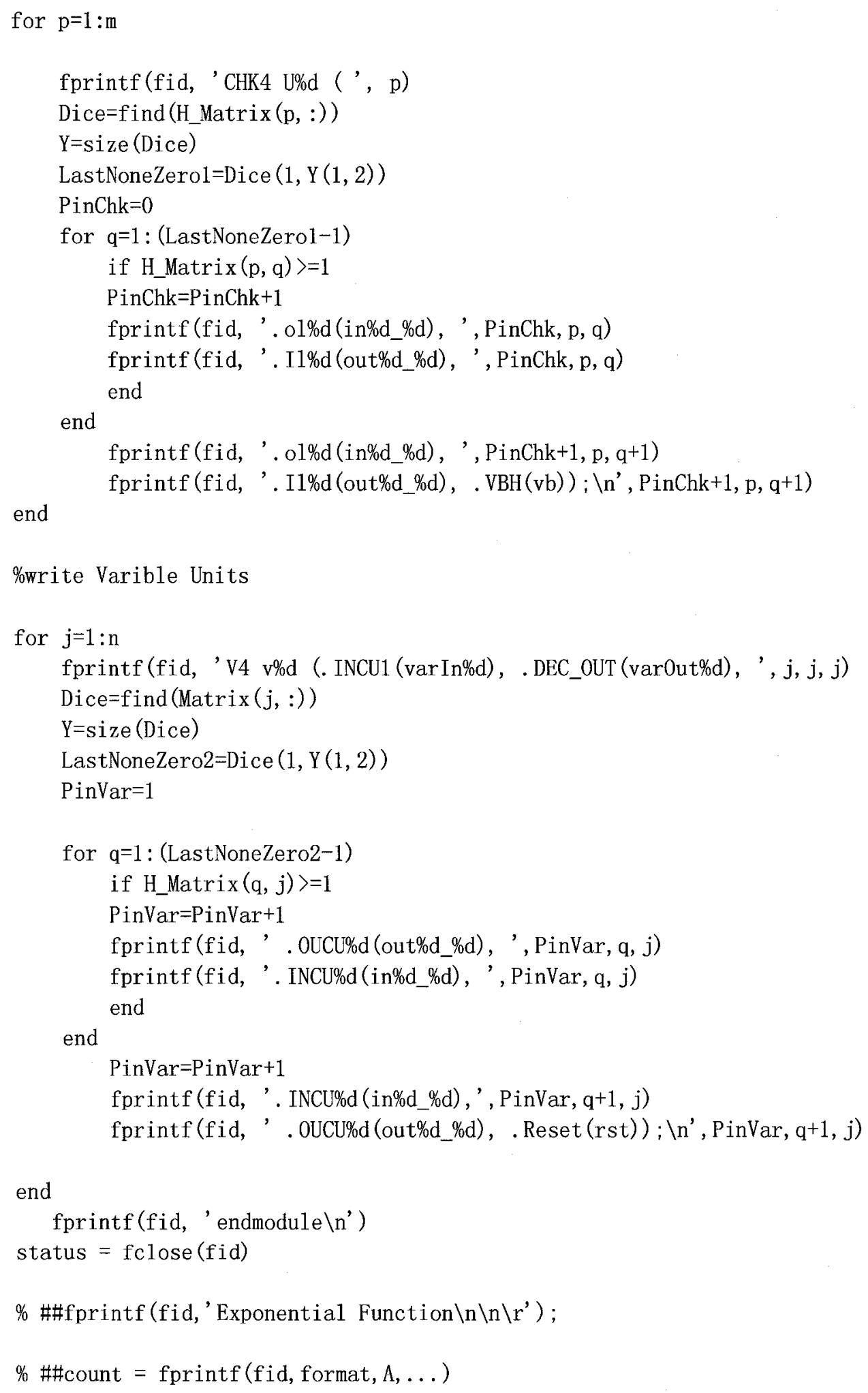




\section{Appendix B Verilog Code Naming Rule}

Naming rule applied in Verilog code generation:

For Variable Nodes:

1) According to the degree, we call the Variable node "Vx". The $x$ represents the degree of the variable node. As the degree increases, the name can be changed to V4, V5, V6, etc.

2) According to the position of the variable node, the unit will be called $v 1, v 2 \ldots v 32 \ldots$

3) All the $\mathrm{I} / \mathrm{O}$ pins in the variable nodes have names beginning with "varOut" as the output signal and "varIn" as the input signal for "varnode1", plus a number representing its position, e.g. varOut1.

4) A special signal (reset signal) for a variable node will be "rst"

For Check Nodes:

1) According to the degree, we call the degree-4 check node CHK4. As the degree increases, the name can be changed to CHK5, CHK6, etc

2) All the I/O pins in the macro cell for the check node are named "outx_y" for the output signal and "inx_y" for the input signal. In addition, $x$ represents the position of the variable node to which the check node connects, while the y represents the position of the check node.

3) A special signal is used to adjust the output impedance of the current mirror is called "VBH".

The internal interconnection wire is represented by $x_{-} y$, since all the inputs and outputs of the check nodes are interconnection wires. In this way, we can describe a decoder systematically even when the cell library expands. For example, a future cell library might include variable node/check nodes of degree $2,3,4,5$ or even more. In that case, the program can still automatically generate Verilog code. 


\section{Appendix C Automatically Generated Verilog Code}

module LDPCdecoder (varOut1, varIn1, varOut2, varIn2, varOut3, varIn3, varOut4, varIn4, varOut5, varIn5, varOut6, varIn6,

varOut7, varIn7, varOut8, varIn8, varOut9, varIn9, varOut10, varIn10, varOut11, varIn11, varOut12, varIn12,

varOut13, varIn13, varOut14, varIn14, varOut15, varIn15, varOut16, varIn16, varOut17, varIn17, varOut18, varIn18,

varOut19, varIn19, varOut20, varIn20, varOut21, varIn21, varOut22, varIn22, varOut23, varIn23, varOut24, varIn24,

varOut25, varIn25, varOut26, varIn26, var0ut27, varIn27, varOut28, varIn28, varOut29, varIn29, varOut30, varIn30,

varOut31, varIn31, varOut32, varIn32, vb, rst );

output varOut1, varOut2, varOut3, varOut4, varOut5, varOut6, varOut7, varOut8, varOut9, varOut10, varOut11, varOut12,

varOut13, varOut14, varOut15, varOut16, varOut17, varOut18,

varOut19, varOut20, varOut21, varOut22, varOut23, varOut24,

varOut25, varOut26, varOut27, varOut28, varOut29, varOut30,

varOut31, varOut32;

input varIn1, varIn2, varIn3, varIn4, $\operatorname{var} \operatorname{In} 5$, varIn6,

varIn7, varIn8, varIn9, varIn10, varIn11, varIn12,

varIn13, varIn14, varIn15, varIn16, varIn17, varIn18,

varIn19, varIn20, varIn21, varIn22, varIn23, varIn24,

varIn25, varIn26, varIn27, $\operatorname{varIn28,} \operatorname{var} \operatorname{In} 29, \operatorname{var} I n 30$,

varIn31, varIn32, vb, rst;

wire out1_7, in1_7, out1_11, in1_11, out1_12, in1_12, out1_19, in1_19,

out2_4, in2_4, out2_18, in2_18, out2_28, in2_28, out2_32, in2_32,

out3_3, in3_3, out3_6, in3_6, out3_9, in3_9, out3_15, in3_15,

out4_1, in4_1, out4_15, in4_15, out4_27, in4_27, out4_31, in4_31,

out5_5, in5_5, out5_20, in5_20, out5_26, in5_26, out5_32, in5_32,

out6_9, in6_9, out6_13, in6_13, out6_14, in6_14, out6_24, in6_24,

out7_5, in7_5, out7_9, in7_9, out7_16, in7_16, out7_23, in7_23,

out8_12, in8_12, out8_18, in8_18, out8_22, in8_22, out8_30, in8_30,

out9_10, in9_10, out9_13, in9_13, out9_18, in9_18, out9_23, in9_23,

out10_10, in10_10, out10_16, in10_16, out10_28, in10_28, out10_30, in10_30,

out11_2, in11_2, out11_6, in11_6, out11_8, in11_8, out11_27, in11_27,

out12_5, in12_5, out12_18, in12_18, out12_28, in12_28, out12_29, in12_29,

out13_11, in13_11, out13_21, in13_21, out13_27, in13_27, out13_29, in13_29,

out14_2, in14_2, out14_14, in14_14, out14_15, in14_15, out14_25, in14_25,

out15_1, in15_1, out15_4, in15_4, out15_8, in15_8, out15_19, in15_19,

out16_14, in16_14, out16_16, in16_16, out16_17, in16_17, out16_26, in16_26,

out17_4, in17_4, out17_7, in17_7, out17_13, in17_13, out17_20, in17_20,

out18_8, in18_8, out18_10, in18_10, out18_24, in18_24, out18_32, in18_32,

out19_22, in19_22, out19_25, in19_25, out19_26, in19_26, out19_29, in19_29, 
out20_2, in20_2, out20_21, in20_21, out20_22, in20_22, out20_31, in20_31, out21_19, in21_19, out21_20, in21_20, out21_30, in21_30, out21_31, in21_31, out22_1, in22_1, out22_6, in22_6, out22_7, in22_7, out22_24, in22_24, out23_3, in23_3, out23_12, in23_12, out23_17, in23_17, out23_21, in23_21, out24_3, in24_3, out24_11, in24_11, out24_23, in24_23, out24_25, in24_25;

CHK4 U1

(.o11(in1_7), .I11(out1_7), .o12(in1_11), .I12(out1_11), .o13(in1_12), . I13(out1_12) , .o14(in1_19), .I14 (out1_19), .VBH (vb));

CHK4 U2

(.o11(in2_4), .I11(out2_4), .ol2(in2_18), .I12(out2_18), .o13(in2_28), . I13(out2_28) , .ol4(in2_32), .I14(out2_32), .VBH (vb));

CHK4 U3

(.ol1(in3_3), .I11(out3_3), .ol2(in3_6), .I12 (out3_6), .o13(in3_9), .I13 (out3_9), .o 14(in3_15),. I14 (out3_15), .VBH(vb));

CHK4 U4

(.o11(in4_1),.I11(out4_1), .o12(in4_15), .I12(out4_15), .o13(in4_27), . I13 (out4_27) , .ol4 (in4_31), .I14(out4_31), .VBH(vb));

CHK4 U5

(.o11(in5_5), .I11(out5_5), .ol2(in5_20), .I12(out5_20), .o13 (in5_26), .I13 (out5_26) , .ol4(in5_32), .I14(out5_32), .VBH(vb));

CHK4 U6

( .o11(in6_9), .I11 (out6_9)，.o12(in6_13)，. I12 (out6_13), .o13(in6_14), . I13 (out6_14) , .o14(in6_24), .I14(out6_24), .VBH(vb));

CHK4 U7

(.o11(in7_5), .I11(out7_5), .o12(in7_9), .I12(out7_9), .ol3(in7_16), . I13(out7_16), .o14(in7_23), . I14(out7_23), .VBH (vb));

CHK4 U8

(.o11(in8_12), .I11(out8_12), .o12(in8_18), .I12(out8_18), .ol3(in8_22), .I13(out8_2 2),.o14(in8_30), .I14(out8_30), .VBH (vb));

CHK4 U9

(.o11(in9_10),.I11(out9_10),.o12(in9_13),.I12 (out9_13), .o13(in9_18), . I13 (out9_1 8), .ol4(in9_23),.I14(out9_23), .VBH(vb));

CHK4 U10

(.ol1(in10_10), . I11 (out10_10), .ol2(in10_16), .I12(out10_16), .ol3(in10_28), .I13(o ut10_28), .o14(in10_30), .I14(out10_30), .VBH(vb));

CHK4 U11

(.o11(in11_2), .I11 (out11_2), .ol2(in11_6), .I12(out11_6), .o13(in11_8), .I13 (out11_ 8), .o14(in11_27), . I14(out11_27), . VBH (vb));

CHK4 U12

(.ol1(in12_5), .I11(out12_5), .ol2(in12_18), .I12 (out12_18), .ol3(in12_28), . I13 (out 12_28), .o14(in12_29), . I14 (out12_29), .VBH(vb)) ;

CHK4 U13

(.ol1(in13_11), .I11(out13_11), .o12(in13_21), .I12 (out13_21), .o13(in13_27), .I13(o ut13_27), .ol4(in13_29), .I14(out13_29), .VBH(vb)); 
CHK4 U14

(.o11(in14_2), .I11(out14_2), .ol2(in14_14), .I12(out14_14), .o13(in14_15), . I13 (out 14_15), .o14(in14_25), .I14(out14_25), .VBH(vb));

CHK4 U15

(.ol1(in15_1), .I11(out15_1), .ol2(in15_4), .I12(out15_4), .o13(in15_8), . I13 (out15_ 8), .o14(in15_19), . I14(out15_19), .VBH (vb));

CHK4 U16

(.o11(in16_14), .I11(out16_14), .o12(in16_16), .I12(out16_16), .ol3(in16_17), . I13(o ut16_17), .o14(in16_26), .I14(out16_26), .VBH(vb));

CHK4 U17

(.ol1(in17_4), .I11(out17_4), .o12(in17_7), .I12(out17_7), .o13(in17_13), . I13(out17 _13), .o14(in17_20), .114(out17_20), .VBH(vb));

CHK4 U18

(.ol1(in18_8), . I11(out18_8), .ol2(in18_10), . I12 (out18_10), .ol3(in18_24), . I13 (out 18_24), .ol4 (in18_32), .I14 (out18_32), .VBH (vb));

CHK4 U19

(.ol1(in19_22),.I11(out19_22), .o12(in19_25), .I12(out19_25), .o13(in19_26),.I13(o ut19_26), .o14(in19_29), .I14(out19_29), .VBH(vb));

CHK4 U20

(.ol1(in20_2), .I11 (out20_2), .o12(in20_21), .I12 (out20_21), .ol3(in20_22), . I13 (out 20_22), .ol4(in20_31), .I14 (out20_31), .VBH (vb));

CHK4 U21

(.ol1(in21_19), .I11(out21_19), .o12(in21_20), .I12(out21_20), .o13(in21_30), .I13(o ut21_30), .o14(in21_31), .I14(out21_31), .VBH(vb)) ;

CHK4 U22

(.o11(in22_1), .I11(out22_1), .o12(in22_6), .I12(out22_6), .o13(in22_7), . I13(out22_ 7), .o14(in22_24), .I14(out22_24), .VBH(vb));

CHK4 U23

(.o11(in23_3), .I11(out23_3), .o12(in23_12),.I12(out23_12), .o13(in23_17), . I13 (out 23_17), .o14(in23_21), . I14(out23_21), .VBH (vb)) ;

CHK4 U24

(.o11(in24_3), .I11(out24_3), .o12(in24_11), .I12(out24_11), .o13(in24_23), . I13 (out 24_23), .o14(in24_25), . I14(out24_25), .VBH(vb)) ;

V4 v1

(. INCU1 (varIn1), .DEC_OUT (varOut1), .oUCU2 (out4_1), . INCU2 (in4_1), . OUCU3 (out15_1), . INCU3 (in15_1), . INCU4 (in22_1), .0UCU4 (out22_1), . Reset (rst));

$\mathrm{V} 4 \mathrm{v} 2$

(. INCU1 (varIn2), . DEC_OUT (varOut2), .0UCU2 (out11_2), . INCU2 (in11_2), .0UCU3 (out14_2 ), . INCU3 (in14_2), . INCU4 (in20_2), .oUCU4 (out20_2), . Reset (rst)) ;

$\mathrm{V} 4 \mathrm{v} 3$

(. INCU1 (varIn3), .DEC_OUT (varOut3), .oUCU2 (out3_3), . INCU2 (in3_3), . ouCU3 (out23_3), . INCU3 (in23_3), .INCU4 (in24_3), .OUCU4 (out24_3), . Reset (rst)) ;

$\mathrm{v} 4 \mathrm{v} 4$

(. INCU1 (varIn4), .DEC_OUT (varOut4), . OUCU2 (out2_4), . INCU2 (in2_4), .0UCU3 (out15_4), 
.INCU3 (in15_4), .INCU4 (in17_4), .0UCU4 (out17_4), . Reset (rst)) ;

V4 v5

(. INCU1 (varIn5), .DEC_OUT (varOut5), .0UCU2 (out5_5), . INCU2 (in5_5), . OUCU3 (out7_5), . INCU3 (in7_5), . INCU4 (in12_5), .0UCU4 (out12_5), . Reset (rst));

V4 v6

(. INCU1 (varIn6), .DEC_OUT (varOut6), .oUCU2 (out3_6), . INCU2 (in3_6), . OUCU3 (out11_6), . INCU3 (in11_6), . INCU4 (in22_6), .0UCU4 (out22_6), . Reset (rst));

$\mathrm{V} 4 \mathrm{v} 7$

(. INCU1 (varIn7), .DEC_OUT (var0ut7), .0UCU2 (out1_7), . INCU2 (in1_7), .0UCU3 (out17_7), . INCU3 (in17_7), . INCU4(in22_7), .OUCU4 (out22_7), . Reset(rst));

V4 v8

(. INCU1 (varIn8), .DEC_OUT (varOut8), . OUCU2 (out11_8), . INCU2 (in11_8), .0UCU3 (out15_8 ), . INCU3 (in15_8), . INCU4 (in18_8), . OUCU4 (out18_8), . Reset (rst));

V4 $\mathrm{v} 9$

(. INCU1 (varIn9), .DEC_OUT (var0ut9), . OUCU2 (out3_9), . INCU2 (in3_9), . ouCU3 (out6_9), . INCU3 (in6_9), . INCU4 (in7_9), .ouCU4 (out7_9), . Reset (rst)) ;

V4 v10

(. INCU1 (varIn10), .DEC_OUT (var0ut10), . oUCU2 (out9_10), . INCU2 (in9_10), . . UUCU3 (out10 _10), . INCU3 (in10_10), . INCU4(in18_10), .0UCU4 (out18_10), . Reset(rst));

V4 v11

(. INCU1 (varIn11), . DEC_OUT (var0ut11)，.OUCU2 (out1_11), . INCU2 (in1_11), .0UCU3 (out13 _11), . INCU3 (in13_11), . INCU4(in24_11), .ouCU4(out24_11), . Reset(rst));

V4 v12

(. INCU1 (varIn12)，.DEC_OUT (var0ut12)， .0UCU2 (out1_12)，. INCU2(in1_12)， . oUCU3 (out8_ 12), . INCU3 (in8_12), . INCU4(in23_12), .oUCU4 (out23_12), . Reset(rst));

$\mathrm{V} 4 \mathrm{v} 13$

(. INCU1 (varIn13), .DEC_OUT (varOut13), . OUCU2 (out6_13), . INCU2 (in6_13), . . OUCU3 (out9_ 13), . INCU3 (in9_13), . INCU4(in17_13), . OUCU4 (out17_13), . Reset(rst)) ;

V4 $\mathrm{v} 14$

(. INCU1 (varIn14)，.DEC_OUT (varOut14)， .OUCU2 (out6_14)，. INCU2 (in6_14)， . OUCU3 (out14 _14), . INCU3 (in14_14), . INCU4 (in16_14), . OUCU4 (out16_14), . Reset (rst));

$\mathrm{V} 4 \mathrm{~V} 15$

(. INCU1 (varIn15), .DEC_OUT (varOut15), . . OUCU2 (out3_15), . INCU2 (in3_15), . . OUCU3 (out4_ 15), . INCU3 (in4_15), . INCU4 (in14_15), . OUCU4 (out14_15), . Reset (rst)) ;

$\mathrm{V} 4 \mathrm{v} 16$

(. INCU1 (varIn16), .DEC_OUT (varOut16), .0UCU2 (out7_16), . INCU2 (in7_16), . OUCU3 (out10 _16), . INCU3 (in10_16), . INCU4 (in16_16), .0UCU4 (out16_16), . Reset(rst));

V4 $\mathrm{v} 17$

(. INCU1 (varIn17)，. DEC_OUT (varOut17)， . OUCU2 (out16_17)，. INCU2 (in16_17)， . INCU3 (in23 _17), . OUCU3 (out23_17), . Reset (rst));

V4 $\mathrm{v} 18$

(. INCU1 (varIn18), .DEC_OUT (var0ut18), . .OUCU2 (out2_18), . INCU2 (in2_18), . . OUCU3 (out8_ 18), . INCU3 (in8_18), .0UCU4 (out9_18), . INCU4 (in9_18), . INCU5 (in12_18), .ouCU5 (out12_ 18), . Reset(rst)); 
V4 v19

(. INCU1 (varIn19), . DEC_OUT (varOut19), .0UCU2 (out1_19), . INCU2 (in1_19), . OUCU3 (out15 _19), . INCU3 (in15_19), . INCU4 (in21_19), . oUCU4 (out21_19), . Reset (rst));

V4 $\mathrm{v} 20$

(. INCU1 (varIn20), .DEC_OUT (varOut20), .OUCU2 (out5_20), . INCU2 (in5_20), .0UCU3 (out17 _20), . INCU3 (in17_20), . INCU4 (in21_20), .0UCU4 (out21_20), . Reset (rst));

$\mathrm{V} 4$ v21

(. INCU1 (varIn21), .DEC_OUT (varOut21), .OUCU2 (out13_21), . INCU2 (in13_21), .0UCU3 (out 20_21), . INCU3 (in20_21), . INCU4 (in23_21), . OUCU4 (out23_21), . Reset (rst)) ;

V4 v22

(. INCU1 (varIn22), .DEC_OUT (varOut22), .0UCU2 (out8_22), . INCU2 (in8_22), . OUCU3 (out19 _22), . INCU3 (in19_22), . INCU4(in20_22), .0UCU4 (out20_22), . Reset(rst));

$\mathrm{V} 4 \mathrm{v} 23$

(. INCU1 (varIn23)，. DEC_OUT (varOut23)， .oUCU2 (out7_23)，. INCU2 (in7_23)， . oUCU3 (out9 23), . INCU3 (in9_23), . INCU4 (in24_23), . OUCU4 (out24_23), . Reset (rst)) ;

V4 v24

(. INCU1 (varIn24), .DEC_OUT (varOut24)， . OUCU2 (out6_24), . INCU2 (in6_24), . OUCU3 (out18 _24), . INCU3 (in18_24), . INCU4(in22_24), .0UCU4 (out22_24), . Reset(rst));

V4 v25

(. INCU1 (varIn25), .DEC_OUT (varOut25), . OUCU2 (out14_25), . INCU2 (in14_25), . OUCU3 (out 19_25), . INCU3(in19_25), . INCU4 (in24_25), .OUCU4 (out24_25), . Reset (rst));

$\mathrm{V} 4 \mathrm{v} 26$

(. INCU1 (varIn26), .DEC_OUT (var0ut26), . OUCU2 (out5_26), . INCU2 (in5_26), . OUCU3 (out16 _26), . INCU3 (in16_26), . INCU4(in19_26), .OUCU4 (out19_26), . Reset (rst));

V4 v27

(. INCU1 (varIn27)，.DEC_OUT (varOut27)， . OUCU2 (out4_27)，. INCU2 (in4_27), .0UCU3 (out11 _27), . INCU3 (in11_27), . INCU4(in13_27), .0UCU4 (out13_27), . Reset(rst));

V4 v28

(. INCU1 (varIn28), .DEC_OUT (varOut28), . OUCU2 (out2_28), . INCU2 (in2_28), . OUCU3 (out10 _28), . INCU3 (in10_28), . INCU4 (in12_28), .ouCU4 (out12_28), . Reset(rst)) ;

V4 $\mathrm{v} 29$

(. INCU1 (varIn29), .DEC_OUT (varOut29), . OUCU2 (out12_29), . INCU2 (in12_29), . OUCU3 (out 13_29), . INCU3 (in13_29), . INCU4(in19_29), . OUCU4 (out19_29), . Reset (rst));

V4 v30

(. INCU1 (varIn30), .DEC_OUT (varOut30), . OUCU2 (out8_30), . INCU2 (in8_30), . OUCU3 (out10 _30), . INCU3 (in10_30), . INCU4(in21_30), . OUCU4 (out21_30), . Reset (rst));

V4 v31

(. INCU1 (varIn31), .DEC_OUT (var0ut31), . OUCU2 (out4_31), . INCU2 (in4_31), . OUCU3 (out20 _31), . INCU3 (in20_31), . INCU4 (in21_31), . OUCU4 (out21_31), . Reset (rst));

V4 v32

(. INCU1 (varIn32), .DEC_OUT (var0ut32), .0UCU2 (out2_32), . INCU2 (in2_32)， .0UCU3 (out5_ 32), . INCU3 (in5_32), . INCU4(in18_32), . OUCU4 (out18_32), . Reset (rst));

endmodule 


\section{Appendix D Partial Technology File}

\# Copyright (C) 1998 Virtual Silicon Technology Inc. . A11 Rights Reserved.

\# Diplomat, Silicon Ready and IP Ambassador are trademarks of Virtual

\# Silicon Technology Inc. .

$\#$

\# Virtual Silicon Technology

\# 1200 Crossman Ave Suite 200

\# Sunnyvale, CA 94089-1116

\# Phone : 408-548-2700

\# Fax : 408-548-2750

\# Web Site : www. virtual-silicon. com

$\#$

\# File Name: header. lef

\# Library Name: vst_n18_sc_tsm_c4

\# Library Release: 1.1

\# Process: Native-18 TSM

\# Generated: $\quad 09 / 29 / 99$ 17:55:10

$\#$

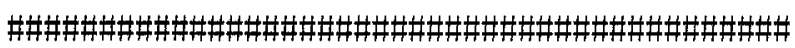

$\#$

\# Modified by Canadian Microelectronics Corporation

\# For CMOSP18 Design Kit

\# March 20, 2000

\#

\# March 20, 2000 B. Veitch

\# - Changed DATABASE MICRONS to 1000 (from 2000)

\# - Changed layer names to match CMOSP18 kit: i. e. from MET1 to meta11 ...

\# MET6 $\rightarrow$ meta16, VIA $\rightarrow$ via12, VIA2 $\rightarrow$ via23 ... VIA5 $\rightarrow$ via56

\# March 21, 2000 R. Wieler

\# - Changed case on turns and generated via arrays

\# March 28, 2000 R. Wieler

\# - Added corner and pad sites

\# Nov. 142000 L. Chen

\# - Added big spacing for wide metals

VERSION 5. 1 ;

NAMESCASESENSITIVE ON ;

UNITS

DATABASE MICRONS 1000 ; 
END UNITS

LAYER metal1

TYPE ROUTING ;

\# ANTENNALENGTHFACTOR 0.001325 ;

WIDTH 0.230 ;

SPACING 0.230 ;

SPACING 0.6 RANGE 1050 ;

PITCH 0.560 ;

DIRECTION HORIZONTAL ;

RESISTANCE RPERSQ 0.078000 ;

CAPACITANCE CPERSQDIST 0.00005059 ;

EDGECAPACITANCE 0.00004653 ;

END meta11

LAYER via12

TYPE CUT ;

END via12

LAYER metal2

TYPE ROUTING ;

\# ANTENNALENGTHFACTOR 0.001325 ;

WIDTH 0.280 ;

SPACING 0.280 ;

SPACING 0.6 RANGE 1050 ;

PITCH 0.660 ;

DIRECTION VERTICAL ;

RESISTANCE RPERSQ 0.078000 ;

CAPACITANCE CPERSQDIST 0.00001832 ;

EDGECAPACITANCE 0.00003675 ;

END metal2

LAYER via23

TYPE CUT :

END via23

LAYER meta13

TYPE ROUTING ;

\# ANTENNALENGTHFACTOR 0.001325 ;

WIDTH 0.280 ;

SPACING 0.280 ;

SPACING 0.6 RANGE 1050 ;

PITCH 0.560 ; 
DIRECTION HORIZONTAL ;

RESISTANCE RPERSQ 0.078000 ;

CAPACITANCE CPERSQDIST 0.000001107 ;

EDGECAPACITANCE 0.00003279 ;

END meta13

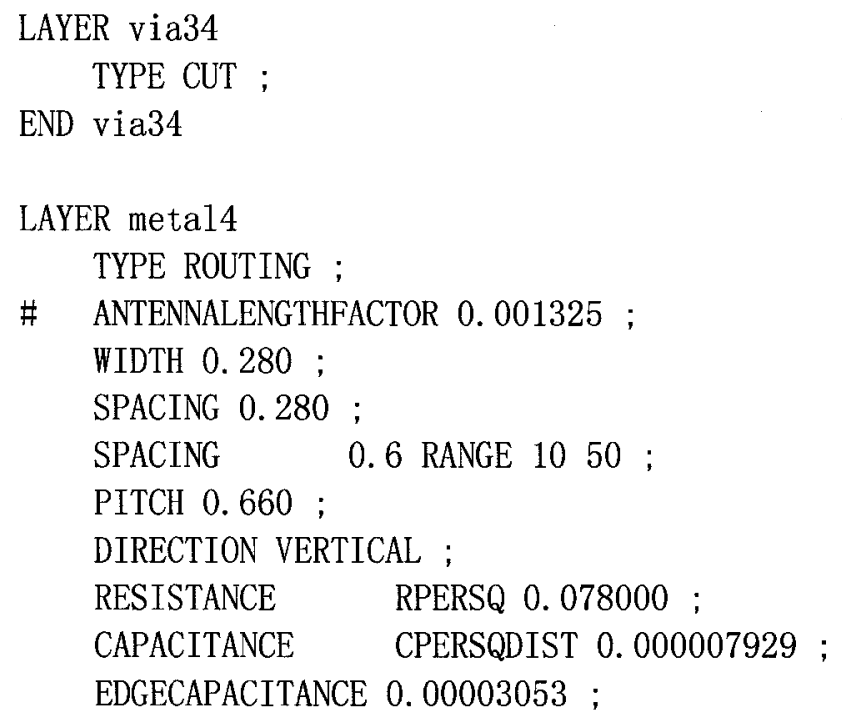


RESISTANCE 8. 100 ;

END VIA3

VIA VIA4 DEFAULT

LAYER metal4 ; RECT $-0.190-0.1400 .1900 .140$;

LAYER via45 ; RECT $-0.130-0.130 \quad 0.130 \quad 0.130$;

LAYER meta15 ; RECT $-0.190-0.140 \quad 0.190 \quad 0.140$; RESISTANCE 8.000 ;

END VIA4

VIA VIA5 DEFAULT

LAYER metal5 ; RECT $-0.240-0.190 \quad 0.240 \quad 0.190$; LAYER via56 ; RECT $-0.180-0.180 \quad 0.180 \quad 0.180$; LAYER metal6 ; RECT $-0.270-0.270 \quad 0.270 \quad 0.270$; RESISTANCE 4.000 ;

END VIA5

VIARULE VIAGEN12 GENERATE

LAYER metal1 ;

DIRECTION HORIZONTAL ; OVERHANG 0.060 ;

METALOVERHANG 0.000 ;

LAYER meta12 ;

DIRECTION VERTICAL ;

OVERHANG 0.060 ;

METALOVERHANG 0.000 ;

LAYER via12 ;

RECT $-0.130-0.130 \quad 0.130 \quad 0.130$; SPACING 0.520 BY 0.520 ;

END VIAGEN12

\section{VIARULE VIAGEN23 GENERATE}

LAYER metal2 ;

DIRECTION VERTICAL ;

OVERHANG 0.060 ;

METALOVERHANG 0.000 ; 
LAYER meta13 ;

DIRECTION HORIZONTAL ;

OVERHANG 0.060 ;

METALOVERHANG 0.000 ;

LAYER via23 ;

RECT $-0.130-0.130 \quad 0.130 \quad 0.130$; SPACING 0.520 BY 0.520 ;

END VIAGEN23

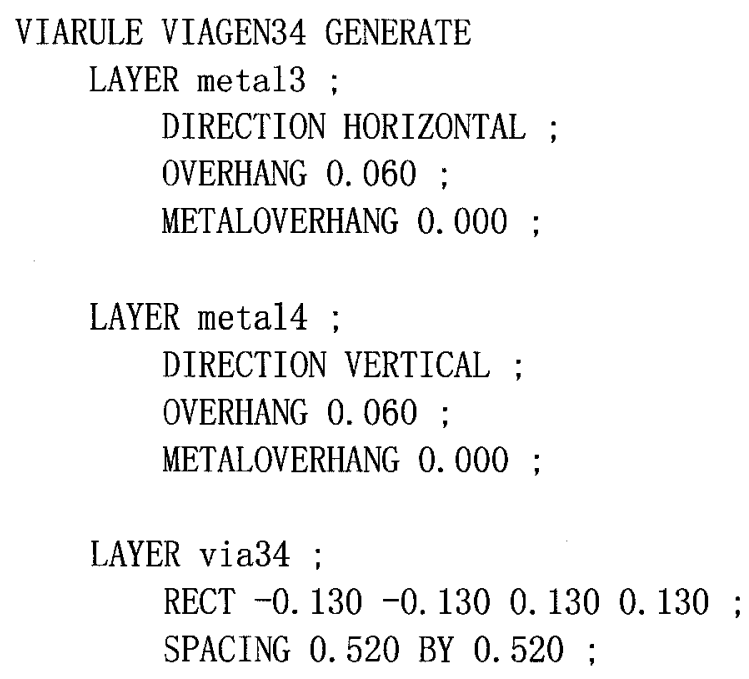

END VIAGEN34

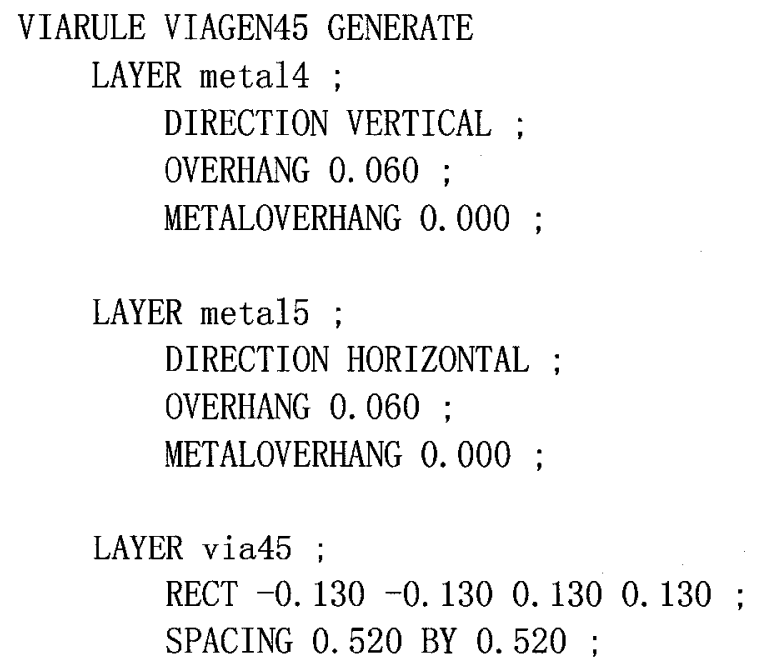

END VIAGEN45

VIARULE VIAGEN56 GENERATE 
LAYER meta15 ;

DIRECTION HORIZONTAL ;

OVERHANG 0.060 ;

METALOVERHANG 0.000 ;

LAYER metal6 ;

DIRECTION VERTICAL ;

OVERHANG 0.090 ;

METALOVERHANG 0.000 ;

LAYER via56 ;

RECT $-0.180-0.180 \quad 0.1800 .180$;

SPACING 0.720 BY 0.720 ;

END VIAGEN56

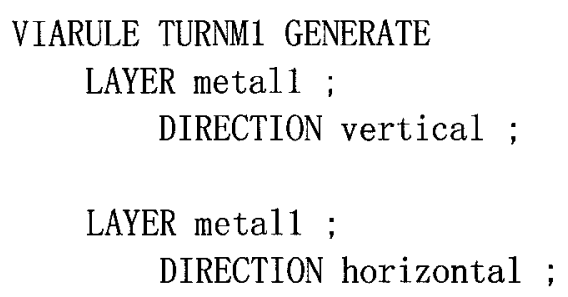

VIARULE TURNM1 GENERATE

LAYER metal1 ;

DIRECTION vertical ;

LAYER metal1 ;

DIRECTION horizontal ;

END TURNM1

\author{
VIARULE TURNM2 GENERATE \\ LAYER meta12 ; \\ DIRECTION vertical ; \\ LAYER meta12 ; \\ DIRECTION horizontal ;
}

END TURNM2 


\section{Appendix E Partial LEF File}

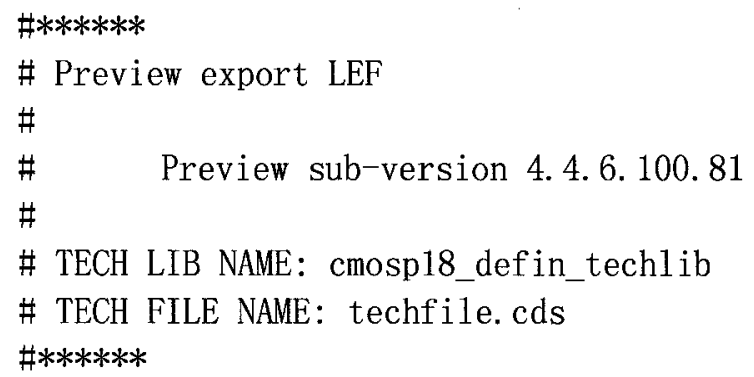

VERSION 5.1 ;

NAMESCASESENSITIVE ON ;

BUSBITCHARS " $\langle>"$;

UNITS

DATABASE MICRONS 1000 ;

END UNITS

SITE core_v4

SYMMETRY Y ;

CLASS CORE ;

SIZE 106. 210 BY 35.580 ;

END core_v4

SITE core_chk4

SYMMETRY Y ;

CLASS CORE ;

SIZE 108.000 By 124.000;

END core_chk4

SITE pad

SYMMETRY $x$ y $r 90$;

CLASS PAD ;

SIZE 0. 100 BY 235.000;

END pad

SITE corner

SYMMETRY x y r90 ;

CLASS PAD ;

SIZE 235.000 BY 235.000; 


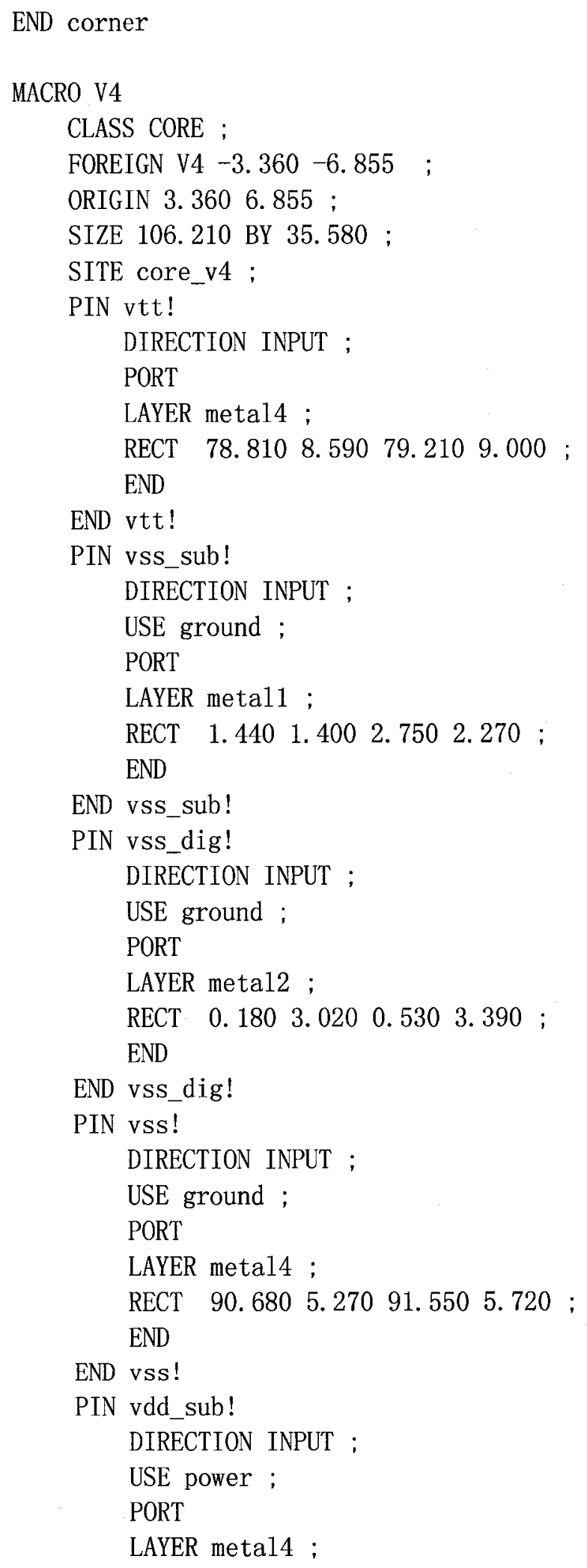




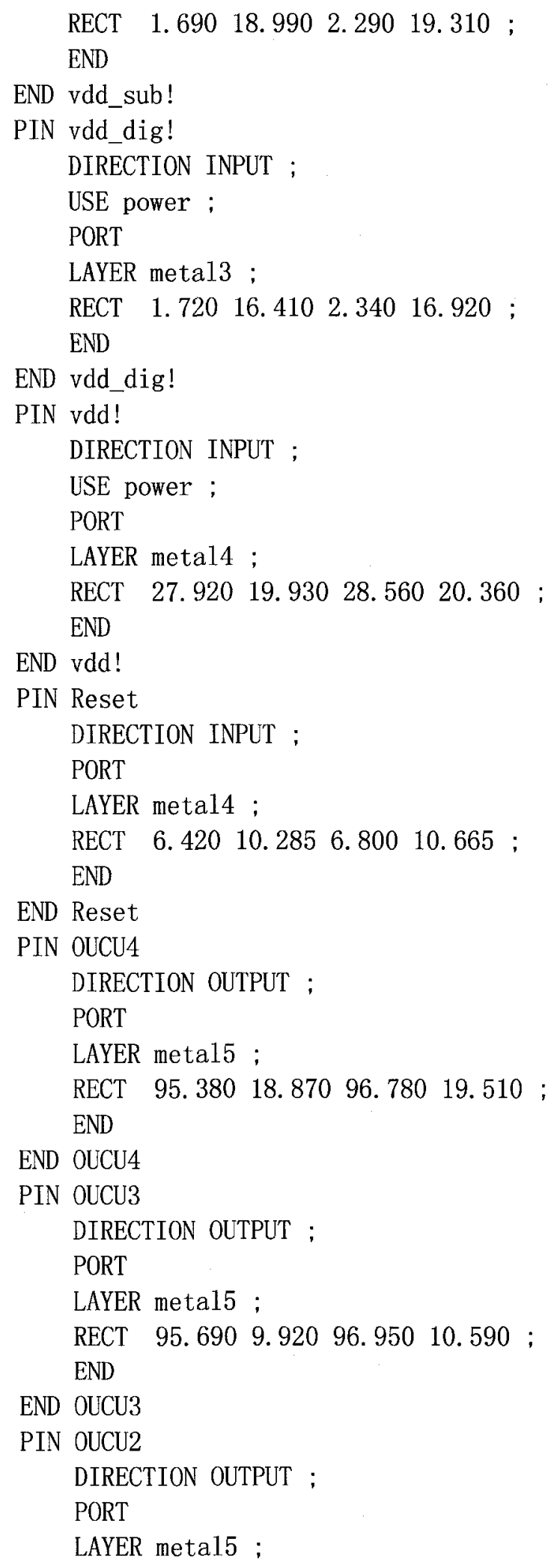


RECT $95.830 \quad 0.980 \quad 97.260 \quad 1.470$;

END

END OUCU2

PIN INCU4

DIRECTION INPUT ;

PORT

LAYER metal3 ;

RECT 23. $160 \quad 18.97023 .900 \quad 19.590$;

END

END INCU4

PIN INCU3

DIRECTION INPUT ;

PORT

LAYER meta13 ;

$\begin{array}{lllll}\text { RECT } & 100.780 & 9.950 & 101.280 & 10.490 \text {; }\end{array}$

END

END INCU3

PIN INCU2

DIRECTION INPUT ;

PORT

LAYER metal3 ;

RECT $21.650 \quad 1.935 \quad 21.9302 .215$;

END

END INCU2

PIN INCU1

DIRECTION INPUT :

PORT

LAYER metal5 ;

RECT $4.340 \quad 1.050 \quad 5.320 \quad 1.560 \quad$;

END

END INCU1

PIN DEC_OUT

DIRECTION OUTPUT ;

PORT

LAYER meta15 ;

RECT $1.180-2.0701 .620-1.630$;

END

END DEC_OUT

OBS

LAYER metal1 ;

POLYGON 93.330 $7.030 \quad 93.300 \quad 7.030 \quad 93.300 \quad 19.825 \quad 93.310 \quad 19.825 \quad 93.310 \quad 21.960$

$\begin{array}{llllllllll}9.730 & 21.960 & 9.730 & 21.900 & 1.400 & 21.900 & 1.400 & 20.330 & 7.290 & 20.330\end{array}$

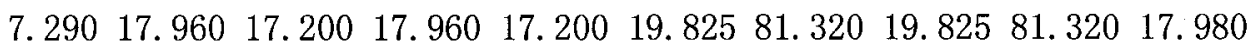

$\begin{array}{lllllllll}90.290 & 17.980 & 90.290 & 15.070 & 89.160 & 15.070 & 89.160 & 14.650 & 92.880\end{array}$

100 
14.650

6. 550

$92.880 \quad 7.200 \quad 81.620 \quad 7.200 \quad 81.620 \quad 14.65085 .340 \quad 14.650 \quad 85.340 \quad 15.070$

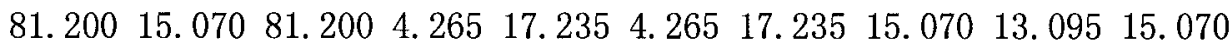

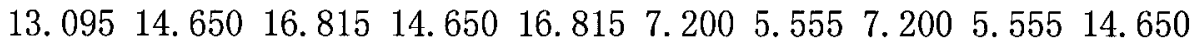

$\begin{array}{lllllllllll}9.275 & 14.650 & 9.275 & 15.070 & 5.135 & 15.070 & 5.135 & 6.780 & 5.140 & 6.780 & 5.140\end{array}$

5. 430

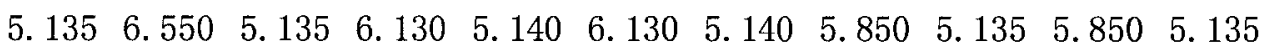

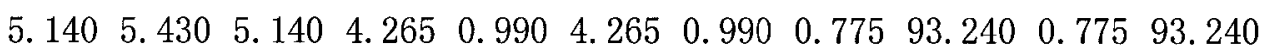

3. 175

93. 3303.175 ;

17.085

$\begin{array}{llllllllll}\text { POLYGON } & 91.155 & 13.830 & 88.490 & 13.830 & 88.490 & 16.405 & 88.500 & 16.405 & 88.500\end{array}$

16. 405

$\begin{array}{lllllllll}88.005 & 17.085 & 88.005 & 13.830 & 86.165 & 13.830 & 86.165 & 16.405 & 88.005\end{array}$

16. 405

16.045

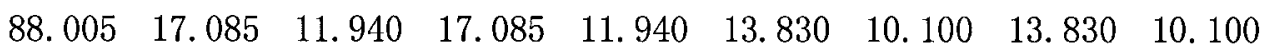

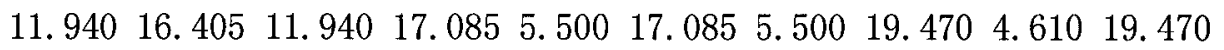

$\begin{array}{llllllllll}4.610 & 19.450 & 4.585 & 19.450 & 4.585 & 18.010 & 4.610 & 18.010 & 4.610 & 17.085\end{array}$

$\begin{array}{lllllllllll}4.570 & 17.085 & 4.570 & 16.405 & 4.610 & 16.405 & 4.610 & 16.390 & 5.500 & 16.390\end{array}$

$\begin{array}{llllllllllll}5.500 & 16.405 & 9.615 & 16.405 & 9.615 & 13.830 & 8.005 & 13.830 & 8.005 & 11.260\end{array}$

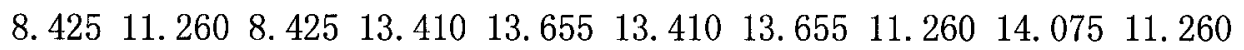

$\begin{array}{lllllllll}14.075 & 13.830 & 12.425 & 13.830 & 12.425 & 16.405 & 34.455 & 16.405 & 34.455\end{array}$

16. 045

11.260

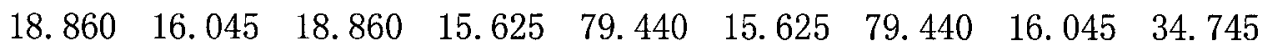

$\begin{array}{lllllllll}34.745 & 16.405 & 85.680 & 16.405 & 85.680 & 13.830 & 84.070 & 13.830 & 84.070\end{array}$

11. 260 ;

$\begin{array}{lllllllll}84.490 & 11.260 & 84.490 & 13.410 & 90.735 & 13.410 & 90.735 & 11.260 & 91.155\end{array}$

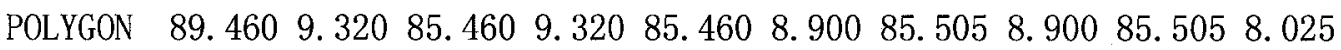

86.4158 .02586 .4158 .90089 .4608 .900 ;

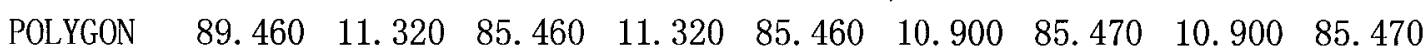

10.040

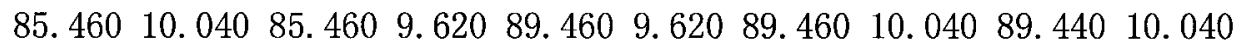
$89.44010 .90089 .460 \quad 10.900$;

12.040

$\begin{array}{llllllllll}\text { POLYGON } & 89.460 & 12.040 & 86.415 & 12.040 & 86.415 & 12.930 & 85.505 & 12.930 & 85.505\end{array}$

$85.460 \quad 12.04085 .460 \quad 11.62089 .46011 .620$;

RECT 86.51014 .65087 .46015 .070 ;

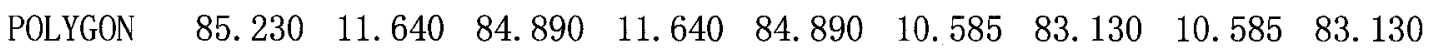

10.930

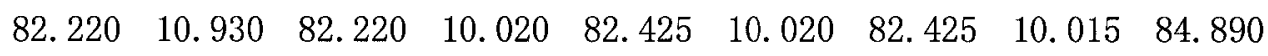

10.015

101 
84. 8909.30085 .2309 .300 ;

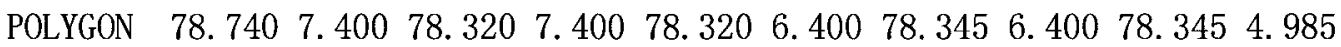

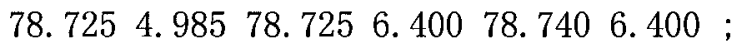

RECT $76.710 \quad 8.31078 .6809 .300$;

$\begin{array}{llllllllll}\text { POLYGON } & 78.140 & 12.860 & 78.120 & 12.860 & 78.120 & 13.895 & 77.740 & 13.895 & 77.740\end{array}$

12.860

$\begin{array}{llllll}77.720 & 12.860 & 77.720 & 10.860 & 78.140 & 10.860 ;\end{array}$

RECT $76.7806 .400 \quad 77.2007 .400$;

RECT $76.780 \quad 10.860 \quad 77.200 \quad 12.860$;

$\begin{array}{lllllllllll}\text { POLYGON } & 76.260 & 12.860 & 75.840 & 12.860 & 75.840 & 9.480 & 75.240 & 9.480 & 75.240 & 6.400\end{array}$

75.6606 .40075 .6609 .06076 .2609 .060 ;

POLYGON 74.960 $7.400 \quad 74.540 \quad 7.400 \quad 74.540 \quad 6.400 \quad 74.565 \quad 6.400 \quad 74.565 \quad 4.985$

$74.945 \quad 4.98574 .945 \quad 6.40074 .9606 .400$;

RECT 73.545 7.630 74.455 8.540 ;

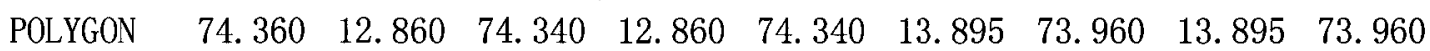

12.860

10.620

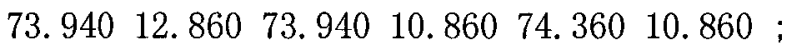

$\begin{array}{llllllllll}\text { POLYGON } & 74.355 & 10.620 & 74.310 & 10.620 & 74.310 & 10.630 & 73.470 & 10.630 & 73.470\end{array}$

73. $445 \quad 10.62073 .4459 .71074 .3559 .710$;

RECT 73.000 6.400 73.420 7.400;

RECT $73.000 \quad 10.86073 .420 \quad 12.860$;

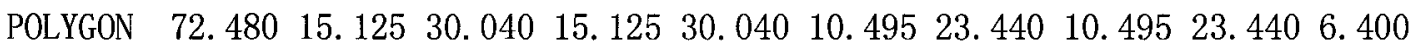

$\begin{array}{llllllllll}23.860 & 6.400 & 23.860 & 10.075 & 30.460 & 10.075 & 30.460 & 14.705 & 55.100 & 14.705\end{array}$

$\begin{array}{lllllllll}55.100 & 13.860 & 57.040 & 13.860 & 57.040 & 14.705 .72 .060 & 14.705 & 72.060 & 9.480\end{array}$

$\begin{array}{llllllllllll}71.460 & 9.480 & 71.460 & 6.400 & 71.880 & 6.400 & 71.880 & 9.060 & 72.480 & 9.060 & \text {; }\end{array}$

POLYGON $\quad 71.180 \quad 7.400 \quad 70.760 \quad 7.400 \quad 70.760 \quad 6.400 \quad 70.785 \quad 6.400 \quad 70.785 \quad 4.985$

$71.165 \quad 4.98571 .1656 .40071 .1806 .400$;

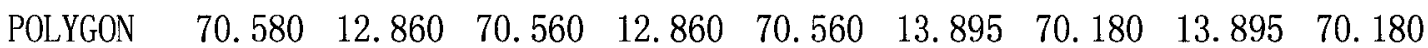

12.860

$\begin{array}{lllllll}70.160 & 12.860 & 70.160 & 10.860 & 70.580 & 10.860 \text {; }\end{array}$

RECT 69.2206 .40069 .6407 .400 ;

RECT $69.22010 .860 \quad 69.64012 .860$; 